UNITED STATES DEPARTMENT OF THE INTERIOR

GEOLOGICAL SURVEY

\title{
Analytical results and sample locality map for rock samples from the Detroit mining district, Juab and Millard Counties, Utah
}

\author{
by \\ D.R.Zimbelman ${ }^{1}$, B.F. Arbogast ${ }^{1}$, C.J. Nutt ${ }^{2}$, \\ Phil Hageman ${ }^{1}$, R.H. Hill ${ }^{1}$, D.L. Fey ${ }^{1}$, and J.H. Bullock Jr \\ Open-File Report 91-319
}

This report is preliminary and has not been reviewed for conformity with U.S. Geological Survey editorial standards and stratigraphic nomenclature. Any use of trade names is for descriptive purposes only and does not imply endorsement by the USGS.

${ }^{1} \mathrm{DFC}$, Box 25046, MS 973, Denver, CO 80225

2DFC, Box 25046, MS 905, Denver, CO 80225 
Studies Related to CUSMAP ..................... 1

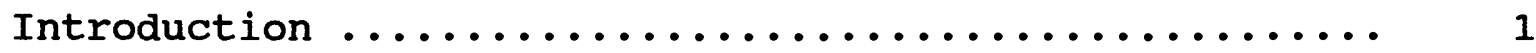

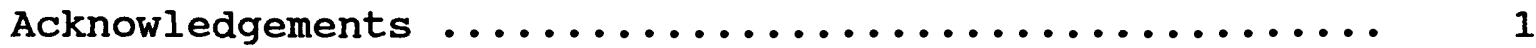

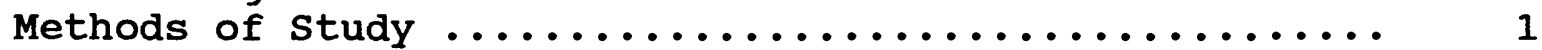

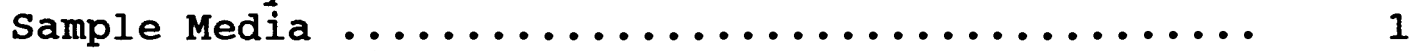

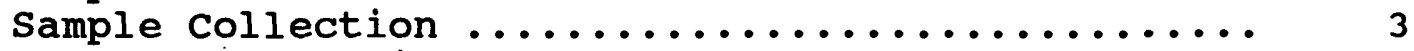

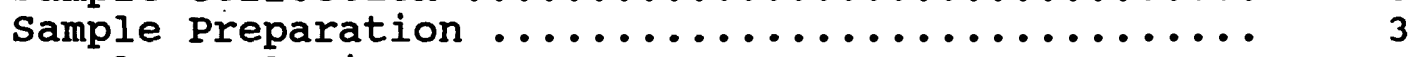

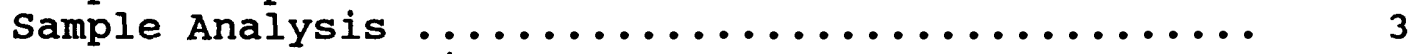

Spectrographic method ................. 3

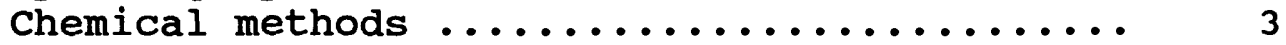

Rock Analysis storage system ..................... 4

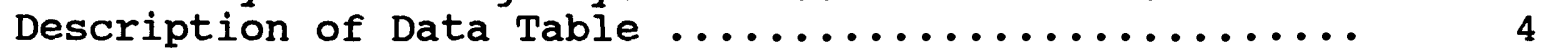

Sample Descriptions .......................... 4

References cited .......................... 5

\section{ILLU8TRATIONB}

Figure 1. Index location $\operatorname{map} \ldots \ldots \ldots \ldots \ldots \ldots \ldots \ldots \ldots$

Plate 1. Sample location map, Detroit minig district.................... in pocket

\section{TABLES}

Table 1. Rock sample numbers for samples collected from selected, named mines and prospects .........

Table 2. Limits of determination for the spectrographic analysis of rocks, based on a 10-mg sample ..

Table 3. Chemical methods and lower limits of determination ....................... 9

Table 4. Results of analyses, Detroit mining district ...............

APPENDIX

Appendix 1. Succinct description of rock samples,

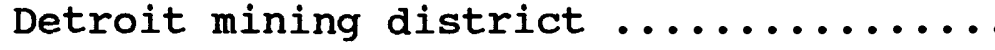




\section{BTUDIES RELATED TO CUBMAP}

This report presents results of analyses from a geochemical survey of the Detroit mining district, Utah. Geochemical samples were collected as one of several multidisciplinary studies associated with the Conterminous United States Mineral Assessment Program (CUSMAP). Geochemical results of analyses for rock samples collected from nearby areas, as well as other rock samples collected from the Detroit district, are in zimbelman and others (1991). Results from heavy-mineral concentrate and stream sediment samples from the Delta $1^{0} \times 2^{0}$ quadrangle are in Arbogast and others $(1990 a, b)$.

\section{INTRODUCTION}

Beginning in 1986 and continuing through 1989, the U.S. Geological Survey conducted a geochemical survey of the Detroit mining district, Juab and Millard Counties, Utah. The study area is in west-central Utah, approximately 20 miles northwest of Delta, and is about $120 \mathrm{mi}$ southwest of Salt Lake City, Utah (fig 1). The Detroit mining district includes the Drum gold mine, from which about 120,000 oz of gold were recovered by open-pit mining methods (Nutt and others, in press).

The geology of the Detroit district and surrounding areas is included in maps and descriptions by Bailey (1974), Lindsey (1979, 1982), Nutt and Yambrick (1989), and Nutt and others (in press). The district is underlain by Proterozoic to Cambrian quartzite and subordinate shale, Cambrian carbonate rocks and subordinate shale, and Eocene to oligocene igneous rocks.

\section{ACKNOWLEDGEMENTS}

Eric Lauha provided rock samples for geochemical analyses, as well as information regarding the genesis of the Drum gold mine and the Detroit mining district, before we were able to get to the field, both of which are greatly appreciated.

\section{METHODS OF STUDY}

\section{sample Media}

Analyses of unaltered or unmineralized rock samples provide background geochemical data for individual rock units. on the other hand, analyses of altered or mineralized rocks, where present, may provide useful geochemical information about the major- and trace-element assemblages associated with a mineralized system. Both types of rock samples were collected and analyzed during this study. 
Figure 1--Index location map of the Detroit mining district, west-central Utah.

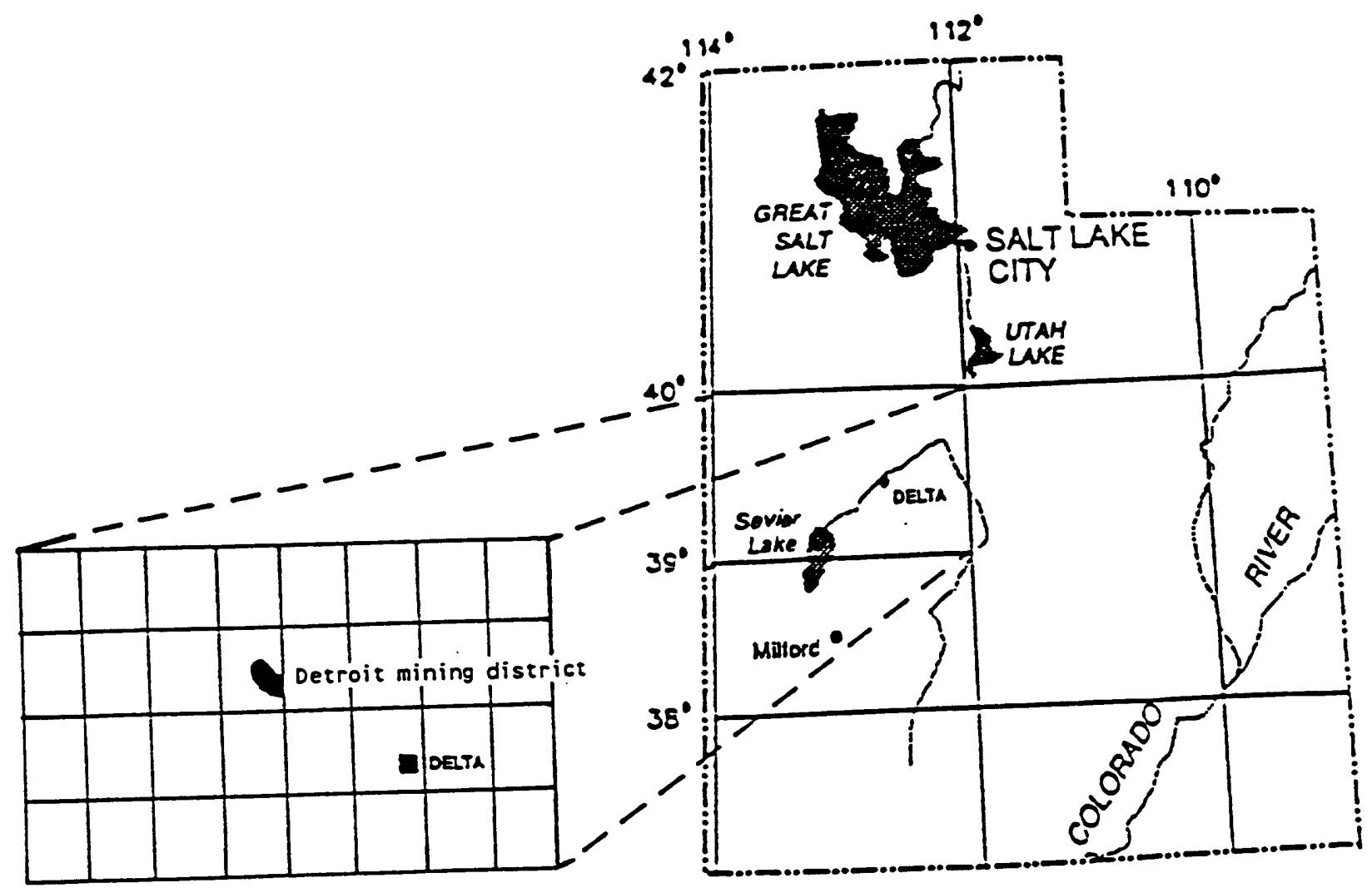

UTAH 
A total of 283 rock samples were collected from the study area (plate 1). Each sample site was assigned a unique fourdigit sample number; where multiple samples were collected at the same site, the samples were assigned suffixes to the four-digit numbers (e.g., 5408A, 5408B etc.). Rock samples were collected from outcrops or mine dumps in the vicinity of the plotted site location. Samples include highly-altered material that was intentionally collected ("high-graded") to gain an understanding of the geochemistry of different types of alteration and mineralization within the mineral systems.

\section{sample Preparation}

Rock samples were crushed and then pulverized to minus $0.15 \mathrm{~mm}$ with ceramic plates.

\section{Sample Analysis}

\section{spectrographic method}

The rock samples were analyzed for 31 (samples collected in 1986 and 1987) or 35 (samples collected in 1988) elements using a semiquantitative, direct-current arc emission spectrographic method (Grimes and Marranzino, 1968). The elements analyzed and their lower limits of determination are listed in table 2 . Spectrographic results were obtained by visual comparison of spectra derived from the sample against spectra obtained from standards made from pure oxides and carbonates. Standard concentrations are geometrically spaced over any given order of magnitude of concentration as follows: 100, 50, 20, 10, and so forth. Samples whose concentrations are estimated to fall between those values are assigned values of $70,30,15$, and so forth. The precision of the analytical method is approximately plus or minus one reporting interval at the 83 percent confidence level and plus or minus two reporting intervals at the 96 percent confidence level (Motooka and Grimes, 1976). Analytical data from the spectrographic analyses are listed in table 4.

\section{Chemical methods}

Other methods of analysis used on samples from the study area are summarized in table 3. Elements analyzed for include $\mathrm{Au}, \mathrm{As}, \mathrm{Sb}, \mathrm{Bi}, \mathrm{Cd}, \mathrm{Zn}, \mathrm{Hg}$, and $\mathrm{F}$. Gold analyses were obtained using an atomic absorption spectroscopy method described by Thompson and others (1968). Arsenic, Sb, Bi, Cd, and Zn were analyzed by an inductively coupled plasma-atomic emission spectrometric method described by crock and others (1987) who report varying precisions for the individual elements that generally range from 2 to 12 percent relative standard deviation. Mercury was analyzed by a modification of the atomic absorption 
method described by Crock and others (1987) who report a precision of between 1.2 and 5 percent relative standard deviation for the method. Fluorine was analyzed by an ion selective electrode method described by Hopkins (1977); precision of this method is generally about eight percent relative standard deviation (Crock and others, 1987). Results of analyses for the rock samples are listed in tables 4 .

\section{ROCK ANALYSIS BTORAGE BYSTEM}

Upon completion of all analytical work, the analytical results were entered into a computer-based file called Rock Analysis storage System (RASS). This data base contains both descriptive geological information and analytical data. Any or all of this information may be retrieved and converted to a binary form (STATPAC) for computerized statistical analysis or publication (VanTrump and Miesch, 1977).

\section{DESCRIPTION OF DATA TABLE}

Table 4 list the results of analyses for the rock samples. The data are arranged so that column 1 contains the USGS-assigned sample numbers. These numbers correspond to the numbers shown on the site location map (plate 1).

Columns in which the element headings show the letter $\mathbf{s}$ below the element symbol are emission spectrographic analyses; aa, icp, ise, or cv represent other methods of analysis, as summarized in table 4. A letter " $N$ " in table 4 indicates that a given element was looked for but not detected at the lower limit of determination shown for that element in table 2 . If an element was observed in the spectrographic analyses but was below the lowest reporting value, a "less than" symbol $(<)$ was entered in table 4 in front of the lower limit of determination. If an element was observed in the spectrographic analyses but was above the highest reporting value, a "greater than" symbol (>) was entered in the table in front of the upper limit of determination. If an element was not looked for in a sample, two dashes (--) are entered in the table in place of an analytical value. Because of the formatting used in the computer program that produced the table some of the elements (Fe, Mg, Ca, Ti, Ag, and $\mathrm{Be}$ ) carry one or more nonsignificant digits to the right of the significant digits. The analysts did not determine these elements to the accuracy suggested by the extra zeros.

Values determined for the major elements ( $\mathrm{Fe}, \mathrm{Mg}, \mathrm{Ca}, \mathrm{Ti}$, $\mathrm{Na}, \mathrm{P}$, and $\mathrm{F}$ ) are given in weight percent; all others are given in parts per million (micrograms/gram).

\section{SAMPLE DESCRIPTIONS}

Succinct descriptions of the samples, with information provided during field examinations, are provided in appendix 1. Where appropriate, appendix 1 is formatted with the following 
information, based on field observations: color, texture, mineralogy, alteration, and other comments.

\section{REFERENCES CITED}

Arbogast, B.F., Folger, H.A., and Zimbelman, D.R., 1990a, Analytical results and sample locality map of heavy-mineralconcentrate samples from the Delta $1^{0} \times 2^{0}$ quadrangle, Tooele, Juab, Millard, and Utah counties, Utah: U.S. Geol. Survey Open-File Report 90-264, $39 \mathrm{p}$.

Arbogast, B.F., zimbelman, D.R., and Whitney, H.A., 1990b, Analytical results and sample locality map of streamsediment samples from the Delta $1^{0} \times 2^{0}$ quadrangle, Tooele, Juab, Millard, and Utah counties, Utah: U.S. Geol. Survey Open-File Report 90-222, 46 p.

Bailey, G.B., 1974, The occurrence, origin, and economic significance of gold-bearing jasperoids in the central Drum Mountains, Utah: Ph.D. dissertation, Stanford University, $300 \mathrm{p}$.

Crock, J.G., Briggs, P.H., Jackson, L.L., and Lichte, F.E., 1987, Analytical methods for the analysis of stream sediments and rocks from Wilderness study Areas: U.S. Geol. Survey OpenFile Report 87-84, 35 p.

Dommer, M.L., 1980, The geology of the Drum Mountains, Millard and Juab Counties, Utah: Brigham Young University Geology studies, v. 27, p. 55-72.

Grimes, D.J., and Marranzino, A.P., 1968, Direct-current arc and alternating-current spark emission spectrographic field methods for the semiquantitative analysis of geologic materials: U.S. Geol. Survey Circular 591, 6 p.

Hopkins, D.M., 1977, An improved ion-selective electrode method for the rapid determination of fluorine in rocks and soils: U.S. Geol. Survey Journal of Research, v. 5, p. 589-593.

Lindsey, D.A., 1979, Geologic map and cross-sections of Tertiary rocks in the Thomas Range and northern Drum Mountains, Juab County, Utah: U.S. Geological survey Miscellaneous Investigations Map I-1176, scale 1:62,500.

Lindsey, D.A., 1982, Tertiary volcanic rocks and uranium in the Thomas Range and northern Drum Mountains, Juab County, Utah: U.S. Geol. Survey Professional Paper 1221, 71 p.

Motooka, J.M., and Grimes, D.J., 1976, Analytical precision of one-sixth order semiquantitative spectrographic analyses: U.S. Geol. Survey Circular 738,25 p.

Nutt, C.J., Thorman, C.H., Zimbelman, D.R., and Gloyn, R.W., in press, Geologic setting and reconnaissance trace-element geochemistry of the Detroit mining district and Drum gold mine, Drum Mountains, west-central Utah: in Geology and ore deposits of the Great Basin, Sparks, Nevada, 1990.

Nutt, C.J., and Yambrick, R.A., 1989, Preliminary geologic map showing igneous-related breccias in Cambrian and older quartzites, Drum Mountains, Utah: U.S. Geol. Survey OpenFile Report 89-099, scale 1:12,000. 
O'Leary, R.M., and Viets, J.G., 1986, Determination of antimony, arsenic, bismuth, cadmium, copper, lead, molybdenum, silver, and zinc in geologic materials by atomic absorption spectrometry using a hydrochloric acid-hydrogen peroxide digestion: Atomic Spectroscopy, 7, p. 4-8.

Thompson, C.E., Nakagawa, H.M., and Van Sickle, G.H., 1968, Rapid analysis for gold in geologic materials, in Geol. Survey research 1968: U.S. Geol. Survey Professional Paper 600-B, p. B130-B132.

VanTrump, George, Jr., and Miesch, A.T., 1977, The U.S. Geol. Survey RASS-STATPAC system for management and statistical reduction of geochemical data: Computers and Geosciences, v. 3, p. 475-488.

Zimbelman, D.R., Arbogast, B.F., Hageman, Phil, Hill, R.H., Fey, D.L., and Bullock, J.H., Jr., 1991, Analytical results and sample locality maps for rock samples collected in and near the Delta $1^{0} \times 2^{0}$ quadrangle, Tooele, Juab, Millard, and Utah Counties, Utah: U.S. Geol. Survey Open-File Report 91$114,256 \mathrm{p}$. 
TABLE 1--ROCK SAMPLES COLLECTED FROM THE VICINITIES OF SELECTED, MINES AND PROSPECTS, DETROIT MINING DISTRICT, UTAH.

MINE OR PROSPECT

Charm mine

\section{SAMPLE SITES}

3083

Drum gold mine, northeast pit $3196,3198,3353,3354,3467,3468$, $3469,3470,3471,3472,3473,3474$, $3476,3477,3478,3479,3480,3481$, $3482,3483,3484,3485,3486,3487$

Drum gold mine, southwest pit 3185, 3186, 3188, 3190, 3191, 3192, $3193,3194,3195,3488,3490,3492$, 3493

Drum gold mine, other areas $3300,3322,3339,3340,3355$

Eldorado mine

3332,3333

Ibex mine

3082

Keystone mine

3291

Martha mine

$3085,3342,3343,3344,3345$ 
TABLE 2.--Limits of determination for the spectrographic analysis of rocks based on a 10-mg sample.

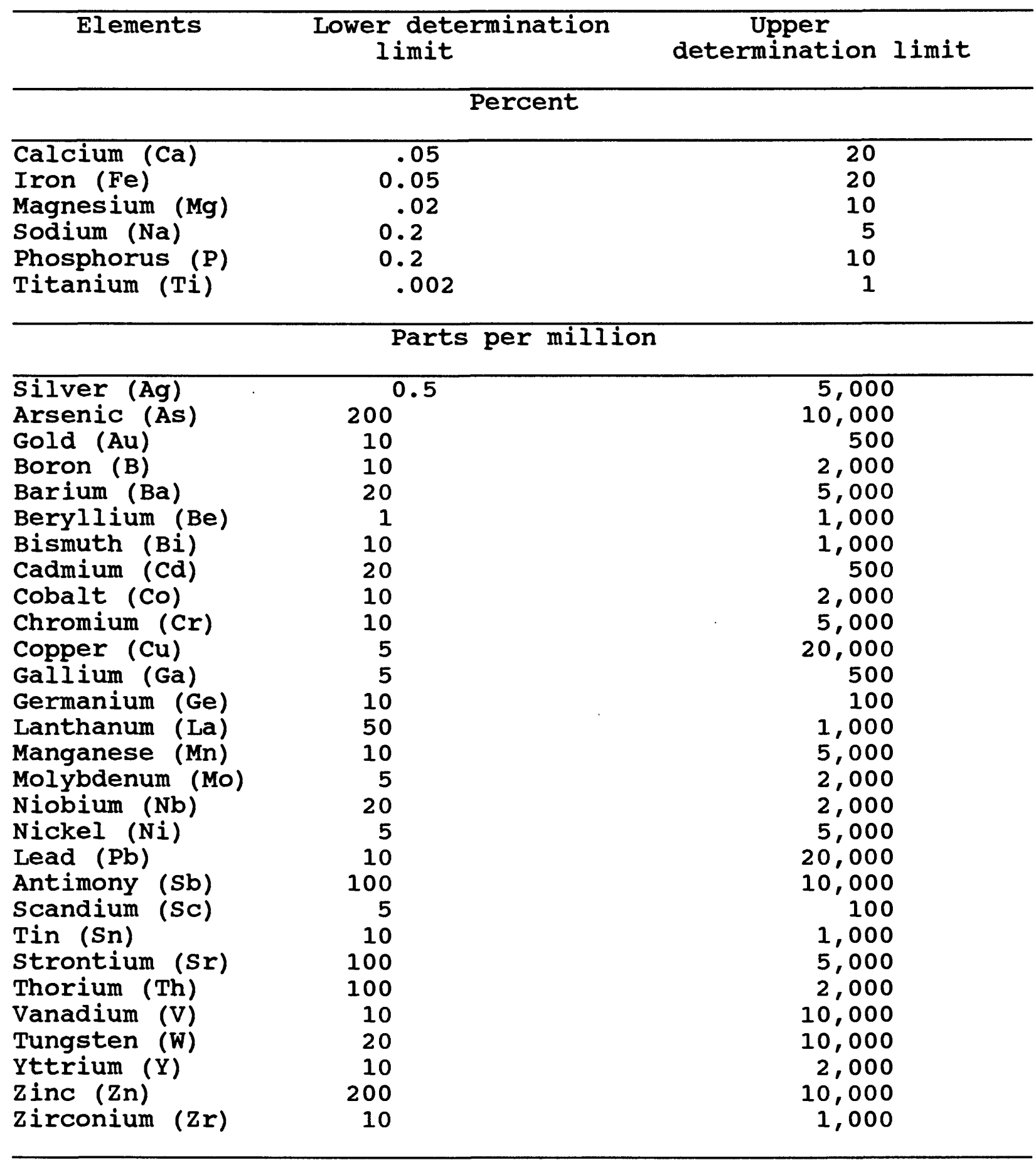


TABLE 3.--Chemical methods and lower limits of determination

[AA, atomic absorption; AACV, atomic absorption cold vapor; ICP, inductively coupled argon plasma-atomic emission spectrographic; ISE, ion selective electrode; all values in parts per million]

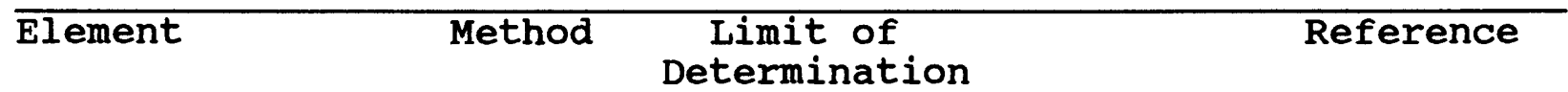

\begin{tabular}{llll}
\hline Gold $(\mathrm{Au})$ & $\mathrm{AA}$ & 0.05 & Thompson and others, 1968
\end{tabular}

Arsenic (As)

Zinc ( $\mathrm{Zn})$

Bismuth (Bi)

Cadmium (Cd)

ICP

ICP

ICP

ICP

ICP

$\mathrm{CV}$

ISE

Fluorine (F)
Antimony (Sb)

$5 \quad$ Crock and others, 1987

2 and modification of

2 O'Leary and Viets, 1986

2

.1

0.02 Crock and others, 1987

100 Hopkins, 1977 
Appendix 1--Succinct descriptions of rock samples collected from the Detroit mining district, Utah. Descriptions are generally formatted as follows: color of fresh rock; texture; mineralogy; and comments. Descriptions are based on field examinations. Abbreviations are: $\mathrm{Cu}=$ copper, $\mathrm{Fe}=\mathrm{iron}, \mathrm{Mn}=\mathrm{manganese}$, $\mathrm{cm}=$ centimeters, $\mathrm{m}=$ meters, $\mathrm{mm}=\mathrm{millimeters.} \mathrm{Samples} \mathrm{marked} \mathrm{*} \mathrm{are}$ from the northeast pit of the Drum gold mine; samples marked ** are from the southwest pit of the Drum gold mine.

SAMPLE

DESCRIPTION

No.

3043A Clay--yellow; very fine grained; clay; altered shale(?) fragment in pebble dike

3043B Porphyry--tan and white; porphyritic; clay-altered plagioclase phenocrysts in fine-grained matrix;

fragment in pebble dike

3043C Pebble dike matrix--white; fine-grained; clay; finegrained porphyry material probably granulated porphyry fragments

3073A silicified carbonate--gray; layered; quartz, pyrite, Fe-oxides; stained yellow, from dump

3073B Jasperoid-gray; microcrystalline, brecciated; quartz, Fe-oxides; stained red and orange

3074A Altered shale--purple; thin-bedded, contorted; clay, Fe-oxides

3074B Altered shale--white; thin-bedded; clay

$3074 \mathrm{C}$ Jasperoid--tan; microcrystalline, brecciated to massive; quartz; black to brown on surface

3074D Limestone--gray; thick-bedded; fine- to medium-grained; calcite cut by calcite veins; country rock near workings

3075 Bleached limestone--white; medium- to coarse-grained; calcite; recrystallized limestone

3076 Breccia--brown to gray; brecciated; angular to rounded quartz fragments, Fe-oxides; purple color on surface

3079

$3080 \mathrm{~A}$ Dolostone--gray; medium-grained; dolomite

Porphyry--Gray; porphyritic; hornblende, plagioclase, biotite phenocrysts, sulfide along fractures

3080B Altered porphyry--green-gray; porphyritic; plagioclase, biotite phenocrysts in clay matrix

3082A Clay + oxides-White, red, purple; white clay set in red to purple matrix; clay, Fe-oxides; probably

porphyritic rock or pebble dike with igneous component

3082B Limestone--Gray; fine-grained; calcite; cut by calcite veins; from mine dump

3082C Jasperoid--Gray; fine-grained, vuggy, brecciated; quartz, Fe-oxides; yellow stain

3083A Iimestone--Gray; fine-grained, calcite; cut by calcite veins; from mine dump

3083B Jasperoid--Gray; brecciated; quartz, Fe-oxides; yellow and red stain 
3083C Altered dike--white with red specks; medium-grained, equigranular; clay, hematite; from mine dump

3084 Jasperoid--tan to red-gray; fine-grained, brecciated; quartz

3085A Jasperoid--tan to pink; brecciated, relict bedding; quartz, Fe-oxides; red to green stain

3085B shaly limestone--yellow; thin-bedded; calcite, clays; from mine dump

3086A Replacement--gray; fine-grained; quartz, disseminated sulfides; replacing carbonate, from mine dump

3086B Porphyry--gray; porphyritic with clay-altered feldspar phenocrysts in fine-grained matrix; clay, sulfide, Feoxides; stained yellow, from mine dump

3086C Mn-oxides--black; fine-grained, massive; Mn-oxides; from Mn mine

3087 Jasperoid--gray; fine- to medium-grained, massive, relict rounded quartz pebbles; quartz; stained black and red, replaced pebble dike

3088A shale--gray; fine-grained, thin-bedded; red-brown on surface

3088B Limestone--Gray; medium-grained, thick-bedded, slightly recrystallized; calcite; cut by calcite veins

3089A Clay zone--gray to purple; fine-grained; clay; probably altered intrusive or shale

3089B Silicified carbonate--gray; medium-grained; quartz, Feoxides; yellow stain

3127 silicified pebble dike--pink and white; brecciated; quartzite pebbles and clay-altered shale fragments in jasperoid (quartz) matrix

3128 Porphyry--green; porphyritic; feldspar, hornblende phenocrysts in green fine-grained matrix

3185A** Altered shale--white to green; fine-grained, contorted; clay, sulfide

3185B** Pebble dike matrix--red-brown; fine-grained; quartz, Fe-oxides; probably granulated quartz pebbles

3186** Altered porphyry--white; porphyritic, clay-altered feldspar phenocrysts in fine-grained matrix; clay, sulfide

3188A** Limestone--gray; fine-grained, thick bedded; calcite; minor calcite veins and yellow stain along fractures

3188B** Limestone--gray; medium-grained, sheared, slightly recrystallized; calcite; minor blebs of yellow stain

$3188 C * *$

$3190 * *$

$3191 A * *$

3191B** Limestone--gray; fine-grained, thin-bedded; calcite Limestone--gray; fine-grained, thin-bedded; calcite, Fe-oxides on surface

Iimestone--gray; fine-grained, thin-bedded; calcite, minor Fe-oxides; yellow stain along fractures and on surface

$3192 * *$

Limestone--red; pisolithic; calcite

Altered carbonate--salt and pepper; medium-grained; carbonate mineral, sulfide, quartz

3193** Limestone--gray; fine-grained, thin-bedded; calcite,

Appendix 1--Description of rock samples 
sulfide along fractures and bedding

shale--green; thin-bedded; green (chlorite?) blebs

$3196 A *$ Jasperoid--green; microcrystalline; quartz

3196B*

shale--green; thin-bedded; sulfide

shale--brown; thin-bedded; altered 196A shale, no visible sulfide

3198* Jasperoid--blue-gray; fine-grained; quartz, sulfide, secondary oxides; stained green-yellow, float

3203 silicified porphyry--gray; porphyritic to fine-grained; quartz, secondary oxides; stained reddish brown

3207 Porphyry--black; porphyritic; fine-grained feldspar, hornblende, biotite phenocrysts in very fine grained matrix

3212 Porphyry--gray green; porphyritic, medium-grained feldspar, hornblende, biotite, quartz phenocrysts in fine-grained matrix; green on weathered surface

Marble--white; medium-grained, recrystallized; calcite

Porphyry-gray; porphyritic, medium-grained feldspar, hornblende phenocrysts in very fine grained matrix

Porphyry--gray; microcrystalline; probably chill margin Porphyry--gray; fine-grained phenocrysts in microcrystalline matrix

Porphyry--green; porphyritic; coarse-grained quartz, feldspar, biotite, hornblende phenocrysts; from prospect pit

3219B clay-altered porphyry--greenish-white with red specks; clay, hematite

3219C Altered rock--green; quartz, Fe-oxides, secondary $\mathrm{Cu}$ minerals; sample pebble size, stained green

3220 Tuff--green-gray; medium-grained; feldspar, quartz, biotite phenocrysts, about $2 \%$ inclusions; chloritized biotite

Clay zone--white; fine-grained; clay; from prospect pit Altered porphyry--green to orange; medium-grained; secondary oxides

3226 Porphyry--green-gray; porphyritic; medium-grained hornblende, feldspar, quartz, biotite phenocrysts in aphanitic matrix

3227 Porphyry--green-gray; feldspar, quartz, biotite phenocrysts in aphanitic groundmass; propylitically altered

3228 Clay zone--white; fine-grained; clay

3229 Jasperoid--gray to red; microcrystalline, relict breccia; quartz, Fe-oxides; stained black to red

3231 Replacement--red, yellow, orange; quartz, Fe-oxides; from prospect pit

3249 kn-oxides-black, yellow; fine-grained, masssive; Mnoxides

3276A Jasperoid--yellow; fine-grained, vuggy; quartz, secondary oxides; cut by thin quartz vein, from prospect pit along fissure in tuff

3276B Jasperoid--red; fine-grained, vuggy; quartz, Fe-oxides; 
from prospect pit along fissure in tuff

3284A Altered porphyry--red; porphyritic; silicified and
clay-replaced phenocrysts; quartz, clay, Fe-oxides;
from breccia complex

Altered porphyry--white; vuggy; clay; from breccia complex

3284B Pebble dike--red and yellow; brecciated; quartzite pebbles, quartz, Fe-oxides

3287A Gouge zone--orange; very fine grained to fine-grained; clay, secondary oxides; along fault

3287B Limestone--gray; medium-grained, massive, slightly vuggy; calcite, dolomite(?); adjacent to fault

3288B Pebble dike matrix--orange to purple; vuggy; clay, Feoxides

3289B Breccia matrix--white, yellow, green; fine-grained, vuggy; clay

3291 Marble--white; medium-grained, massive, recrystallized limestone; calcite

3292B Porphyry--gray-green; porphyritic; coarse-grained feldspar, hornblende phenocrysts in very fine-grained matrix; propylitically altered

3294B Clay--white, yellow, red; fine-grained; clay, Feoxides; altered shale

3294C Replacement--yellow, orange; fine-grained; Fe-oxides, clay

shale-brown; thin-bedded; unaltered

Marble--white; medium- to coarse-grained, recrystallized limestone; calcite

3298

3299

3300

$3302 \mathrm{~A}$

shale--green, brown; thin-bedded; not altered

Altered porphyry--white, yellow; porphyritic; quartz, clay

3302B Pebble dike--yellow, white; vuggy; quartzite, porphyry pebbles in clay, secondary oxide matrix

3303 Pebble dike matrix--gray; fine to medium-grained;

quartz

3305 Pebble dike, igneous matrix--yellow, purple, white;

brecciated; clay, quartz, Fe-oxides; partially

silicified

3306 Quartzite--fine to medium-grained; quartz; black stain, from shear zone

3307A Pebble dike, igneous material--yellow; porphyritic; clay, secondary oxides

3307B Pebble dike, quartzite--gray to yellow; fine-grained; quartz; black stain

3319A Pebble dike--gray; brecciated; quartz, Fe-oxides; red stain

3319B Pebble dike--gray; brecciated; quartz, Fe-oxides; red, yellow stain

3322 Quartzite--red, purple; fine-grained; quartz, hematite

Appendix 1--Description of rock samples 
3323

$3325 A$

$3325 B$

$3325 \mathrm{C}$

3325D

$3326 A$

$3326 \mathrm{~B}$

$3326 C$

3327A

3327B

$3327 C$

$3327 D$

$3328 A$

$3328 B$

$3328 \mathrm{C}$

$3328 D$

$3328 E$

$3329 A$

3329B

$3329 C$

3330A

$3330 \mathrm{~B}$

$3330 \mathrm{C}$

$3330 D$

3330E

$3331 \mathrm{~A}$

Quartzite--red, purple; fine-grained; quartz, hematite Altered porphyry--gray; porphyritic; clay altered phenocrysts; from adit

clay breccia matrix--orange to purple; fine-grained; clay, secondary oxides; from adit

Jasperoid--gray; massive, vuggy, fine-grained; quartz, Fe-oxides; stained yellow, from dump

Quartzite--gray to white; fine-grained, fractured; quartz, secondary oxides; yellow, blue, black stain, float

Jasperoid--gray; massive, vuggy, microcrystalline; quartz, Fe-oxides; stained red, vugs filled with euhedral quartz

Jasperoid--gray; thinly banded, microcrystalline; quartz, Fe-oxides; red stain

Iimestone--gray; thick-bedded, silty layers; calcite; calcite veins along fractures

Jasperoid--gray; microcrystalline, sheared; quartz; red-brown on surface; from prospect

Recrystallized limestone--gray and white; mediumgrained, layered; calcite; from prospect dump

Altered rock--red, brown; fine-grained, sheared; Feoxides; probably shale, from prospect

Replacement--yellow; fine to medium-grained; secondary oxides; from prospect pit

Oxides--Black; microcrystalline, frothy; oxides; from mine workings

Oxides--yellow; fine-grained, friable; oxides; from mine workings

Recrystallized limestone--white; medium-grained, thinly bedded, calcite

Limestone--gray; medium-grained, thick-bedded; calcite; country rock, not visibly altered

Oxides--purple to yellow; fine-grained, frothy; oxides; from dump of prospect pit

Jasperoid--gray to tan; microcrystalline, massive to brecciated; quartz; secondary $\mathrm{Cu}$ minerals; green, blue stain; from mine

Replacement--brown; fine-grained, vuggy; secondary oxides, clay; from mine

Recrystallized limestone--white; medium-grained, brecciated; calcite; from mine

Jasperoid--gray, tan red; fine- to medium-grained, brecciated to massive; quartz, Fe-oxides

Gouge--white, yellow; fine-grained; clay, secondary oxides

Appendix 1--Description of rock samples 
oxides; from mined fault zone

$3331 \mathrm{C}$

$3331 D$

$3332 \mathrm{~A}$

$3332 B$

$3332 \mathrm{C}$

$3332 \mathrm{D}$

$3332 E$

$3332 F$

3333

$3334 \mathrm{~A}$

$3334 \mathrm{~B}$

3336

3337A

$3337 B$

3339A

$3339 B$

3340

3342

3343

3344

3345

$3346 \mathrm{~A}$

$3346 B$

$3346 C$

oxides--red, yellow; fine-grained, massive, frothy, friable; Fe-oxides from mined fault zone

Carbonate vein--coarse-grained, euhedral

Jasperoid--gray, pink; fine-grained, massive; quartz, Fe-oxides; overlies mine workings

Iimestone--gray; fine- to medium-grained, thin- to thick-bedded; calcite

Dolostone--white; recrystallized; dolomite; from mine area

clay gouge zone--white, yellow; fine-grained; clay from mine

oxides-yellow, red, black; fine-grained, massive, frothy; secondary oxides; from mine

Recrystallized limestone--white; medium-grained, vuggy; calcite; unoxidized ore

Jasperoid--gray green; microcrystalline, massive; quartz; from mine area

Jasperoid--gray, pink; microcrystalline to finegrained, massive to thinly layered; quartz, Fe-oxides; orange to red-brown stain

Jasperoid--gray, pink; microcrystalline to finegrained, massive to brecciated; quartz; jasperoid overlying mine workings

clay--white, yellow; fine-grained; clay, Fe-oxides;

from beneath jasperoid, similar to ore zone

Quartzite--gray; fine-grained, shattered; quartz;

yellow and red stain along fractures

Quartzite--red, purple; fine- to medium-grained; quartz, Fe-oxides

Quartzite--yellow; fine- to medium-grained, vuggy; quartz, Fe-oxides; float

Quartzite--white, yellow, red; thin-bedded; quartzite, Fe-oxides; along fault

Dolostone--gray; medium-grained; dolomite; Tatow member of Pioche Formation

Jasperoid--gray; microcrystalline, banded; quartz;

float at prospect

Recrystallized limestone--white; medium- to coarsegrained; calcite

Recrystallized limestone--white; coarse-grained; calcite

Altered limestone--tan; fine-grained, thin-bedded; calcite, dolomite(?); brown on outcrop

Limestone--gray to white; fine-grained, slightly recrystallized; calcite

Jasperoid--red; very fine grained, massive, slightly vuggy; quartz, Fe-oxides; float at prospect pit Jasperoid--gray; very fine grained, vuggy, sheared; quartz, secondary oxides; orange stain, from dump Fe-oxides-black, purple; fine- to coarse-grained, vuggy; Fe-oxides; from dump

Appendix 1--Description of rock samples 
3346D Dolostone--gray; medium-grained; dolomite; brown on outcrop, country rock

3347 Jasperoid--gray, red, orange; vuggy, cut by calcite veins; quartz, Fe-oxides; float

3348A Porphyry--green gray; porphyritic, sheared; mediumgrained feldspar, biotite phenocrysts in aphanitic matrix, yellow oxide blebs

3348B Replacement--yellow; fine-grained, vuggy, leached; secondary oxides; from prospect pit

3349 Carbonate--brecciated; carbonate minerals, secondary oxides; cut by thin calcite veins

3350A Altered carbonate--yellow; fine-grained, vuggy, leached; carbonate minerals, secondary oxides; from mine

3350B Clay zone--white, red, yellow, orange; fine-grained; clay, Fe-oxides; from mine

3350C Mn-oxides--black; fine- to medium-grained, vuggy, massive; Mn-oxides; from mine

3351 Jasperoid--red; microcrystalline, massive; quartz, Feoxides

3353* Jasperoid--white; microcrystalline; fragment in pebble dike; quartz; red to green on surface

Altered igneous rock--White; fine-grained; clay

Quartzite--black; fine-grained; quartz

Quartz vein--white; subparallel to bedding; quartz,

3369

3377

3378

3379

3380

3381 Oxides from pebble dike--black; frothy; oxides; matrix minor oxide staining along fractures

Quartzite--red; brecciated; quartz

Quartzite--red; fine-grained, brecciated; quartz

Fe-oxides-black; frothy, massive; Fe-oxides; float Porphyry--white; porphyritic; clay

Red stained gouge from pebble dike--red; fine-grained; quartz, Fe-oxides material

3385 Altered porphyry--gray; porphyritic; clay, quartz; float

Quartzite breccia--red; brecciated; quartz, Fe-oxides

stained pebble dike in quartzite--red to black;

3390A Jasperoid--green; microcrystalline; quartz; inclusions in quartzite breccia

3390B Oxide coating--black; fine-grained; oxides; coating on fractures

3390C Quartzite breccia--white; brecciated; quartz; rehealed breccia

3394 Oxide-coated rock along fault--white; oxides, clay; yellow on surface, probably altered igneous rock

3398 Oxide-coated quartzite--black; fine-grained; quartz, oxide; shattered quartzite

3406 Jasperoid--tan; microcrystalline, massive; quartz

3408 Jasperoid--tan; microcrystalline, massive; quartz, black oxide blebs 
Jasperoid--gray-green; microcrystalline, massive; quartz; slight black stain

3410 Altered porphyry--gray-green; medium-grained, porphyritic; quartz, plagioclase phenocrysts;

3411

3413

$3467 A *$

$3467 \mathrm{~B} *$

$3468 B *$

$3468 C *$

$3469 *$

$3470 A *$

$3470 B *$

$3470 \mathrm{C*}$

$3471 *$

$3472 \mathrm{~A} *$

$3472 \mathrm{~B} *$

3473A*

$3473 B$ *

$3473 C *$

3473D*

$3473 \mathrm{E}$ *

3473F*

3473G*

$3474 \mathrm{~B}$ *

$3474 \mathrm{C}$ *

3476*

3477 *

3478 *

3479 *

3480 * silicified, blue stain

Oxide--black; fine-grained, frothy; oxides

Jasperoid--yellow; microcrystalline; quartz; along fault in quartzite

Jasperoid--tan; microcrystalline; stained red-brown; quartz; early jasperoid

Pebble dike--white to yellow; brecciated; clay, Feoxide; cuts jasperoid in $3467 \mathrm{a}$

Gouge--orange; fine-grained; secondary oxides; in Tatow member of Pioche Formation

clay--fine-grained; in shear zone; clay; altered shale (?)

Tuff--yellowish green; porphyritic; large muscovite grains; slightly altered

Altered igneous rock--white to purple; friable; clay, quartz; in breccia zone

Jasperoid--gray; microcrystalline; quartz; along highangle fault, gray to pink on surface

Jasperoid--gray; microcrystalline; brecciated; quartz; along high-angle fault, black on surface

Jasperoid--gray; microcrystalline; quartz; along highangle fault

clay zone--microcrystalline; breccia zone

Pebble dike matrix--granulated carbonate

Pebble dike matrix--white, orange; fine-grained, liesegang banding; clay, secondary oxides

Jasperoid--gray; microcrystalline, brecciated; quartz, Fe-oxides; stained red

Quartz-rich rock--gray; fine-grained quartz with

disseminated clay blebs; pebble dike(?)

Porphyry fragment in pebble dike--gray to white; porphyritic; clay

Jasperoid--green; microcrystalline, fractured; quartz Jasperoid--black; microcrystalline, fractured; quartz Jasperoid--red; microcrystalline, fractured; quartz oxide--brown to orange; fine-grained, sheared; oxides; in fault zone

clay-altered shale--white; fine-grained; clay; lower member of the Pioche Formation

clay-altered igneous rock--white, red, orange; finegrained; clay, Fe-oxides; in Tertiary conglomerate Jasperoid--black, red; microcrystalline; quartz

Altered carbonate--brown, red, orange; friable, rubbly; oxides, carbonate

Oxide replacement--black; fine-grained; oxides; in lowermost carbonate unit

clay-altered shale--gray, orange; fine-grained; clay, sericite; lower member of the Pioche Formation 
3481* Jasperoid--gray; microcrystalline; red-brown on surface; quartz

3482* Clay-altered felsite--white; fine-grained, porphyritic; quartz phenocrysts, clay and quartz matrix

3483* Jasperoid--black; fine-grained; quartz; black to redorange on surface, cuts and replaces felsite

3484* Oxide replacement--red-brown; earthy; oxides, cut by calcite veins; replaces lowermost carbonate unit

3485* Oxide replacement--red-brown; earthy; oxides; replaces of lowermost carbonate unit

3486* Shale--green-yellow; fine-grained; clay, sulfide; lower member of the Pioche Formation

3487* Clay-altered igneous rock--white, red; fine-grained; clay, Fe-oxide

3488B** Pebble sill--light gray to purple; fine- to mediumgrained, friable; quartz, clay; in ore zone

3488E** Ore--red, white; thin-bedded; clay, hematite; replaces sulfide-altered shale

3488F** Clay material from pebble sill--gray; friable; clay; probably broken fragment

3488G** Clay-altered rock--white; friable; clay; probably altered carbonate rock

3490** Pebble sill--gray; granulated; quartz

3492** Replaced carbonate--white; fine- to medium-grained; clay, quartz

3493A** Clay-altered porphyry fragment--white, porphyritic; clay; fragment from pebble sill

$3493 B * *$ shale--yellow; fine-grained, sheared

$3493 C * *$ Jasperoid--red-brown; microcrystalline; quartz; fragment in pebble sill

3493D** Jasperoid--black; microcrystalline; quartz; fragment in pebble sill

$3493 \mathrm{E} * *$

$3493 \mathrm{~F} * *$

Jasperoid--green; microcrystalline; massive; quartz

Jasperoid--yellow and red; brecciated; quartz, Feoxides; replaces carbonate(?)

3493G** Pebble sill--gray; flow banded; composed of granulated quartz and clay-altered fragments

$3493 \mathrm{H} * *$

3500

3501

Jasperoid--red to black; brecciated; quartz

Quartz vein--white; cutting quartzite

Pebble dike--red; brecciated; quartzite matrix, Feoxides

3502 Pebble dike--red, purple; brecciated; quartz matrix, Fe-oxides

3503 Hematite breccia in quartzite--red; brecciated; Feoxides; along fault

3504 Pebble dike--in quartzite; brecciated; quartz matrix; pebble dike along fault

3505 Pebble dike-red; brecciated; quartz, Fe-oxides; in high shattered and brecciated quartzite

3506 Clay zone--red; fine-grained; clay, Fe-oxides; along fault

3507 Quartzite--red; black; brecciated; quartz, Fe-oxides

Appendix 1--Description of rock samples 
3544

3545

3546

3547

3548

3549

3550

Quartzite--red; brecciated; quartz, Fe-oxides Quartzite--red; brecciated; quartz, Fe-oxides Altered porphyry--gray; porphyritic; partially silicified

oxide--black; oxides; coating on fractured quartzite Altered rock--yellow, red; Fe-oxides; alteration zone along fault

Altered carbonate--at intersection of fault and quartzitelcarbonate contact

Pebble dike--in quartzite; brecciated; quartz matrix Quartzite--red; brecciated; quartz, Fe-oxides Quartzite--red; brecciated; quartz, Fe-oxides Gouge--red; Fe-oxides; from fault zone

Pebble dike--red; brecciated; clay, Fe-oxides; igneous matrix, from prospect pit

Quartzite--red; quartz, Fe-oxides; from prospect pit Pebble dike--igneous matrix; quartz

Jasperoid--gray; microcrystalline; quartz, Fe-oxides Pebble dike--red; igneous and quartzite clasts, Feoxides

Porphyry--altered; igneous rock mixed with brecciated quartzite

Jasperoid--gray, tan; quartz

Porphyry--altered

Jasperoid--gray, tan; microcrystalline, relict breccia; quartz, Fe-oxides along fractures

Porphyry--altered

Porphyry--altered

Jasperoid--brecciated; quartz

Porphyry--silicified, quartz

Breccia--red; brecciated; quartz, Fe-oxides, margin of breccia dike

Breccia pipe--altered matrix material

Pebble dike matrix--red; quartz, Fe-oxides

Breccia complex--brecciated; quartz, Fe-oxides; stained yellow and red

Gouge--red; along fault within breccia complex; quartz, Fe-oxides

Pebble dike--quartz, Fe-oxides

Altered pebble dike--igneous, shale, quartzite clasts Quartz vein--white, red; quartz, Fe-oxides

Igneous rock--altered; along fault

clay--orange; clay, Fe-oxides; fills fractures in quartzite

clay--orange; clay, Fe-oxides, quartz; from prospect pit

Quartzite--quartz, Fe-oxides; red stained

Pebble dike matrix--quartz, Fe-oxides; red stain Quartzite--quartz, Fe-oxides; red stain; next to fault Igneous rock--altered

Pebble dike--tan, purple; quartz matrix

clay--white; clay; altered igneous rock; red stain

Appendix 1--Description of rock samples 
Porphyry--gray; purple stain; from prospect pit Gouge--yellow, brown; quartz, secondary oxides; along fault

Gouge--red, yellow; friable; clay; along fault, from prospect pit

Altered shale--yellow, red, brown; along fissure

Altered carbonate-yellow, red, black; oxides, quartz, calcite; along fault

3556

clay--along low-angle structure; clay, oxide; from prospect

Altered carbonate--oxide

3558

Altered carbonate--oxides, calcite veins

3559

Altered porphyry--purple; clay, quartz; cut by hematite veins 
TABLE 4--RESULTS OF ANALYSES, DETROIT MINING DISTRICT, UTAK

[N, not detected; <, detected but below the limit of determination shown; >, determined to be greater than the value shown.]

\begin{tabular}{|c|c|c|c|c|c|c|c|c|c|c|c|}
\hline Sample & Lat i tude & Long & i tude & $\begin{array}{c}\text { Fe-pct } \\
\text { s }\end{array}$ & $\begin{array}{c}\text { Mg-pct } \\
\text { s }\end{array}$ & $\begin{array}{c}\mathrm{Ca}-\mathrm{pct} \\
\mathrm{s}\end{array}$ & $\underset{s}{T i-p c t}$ & $\begin{array}{l}\text { Mn-ppm } \\
s\end{array}$ & $\begin{array}{c}\text { Ag-ppm } \\
s\end{array}$ & $\begin{array}{c}\text { As-ppm } \\
s\end{array}$ & $\begin{array}{l}\text { Au-ppm } \\
s\end{array}$ \\
\hline $\begin{array}{l}80 H 3043 A \\
80 H 3043 B \\
80 H 3043 C \\
70 H 3073 A \\
70 H 3073 B\end{array}$ & $\begin{array}{lll}39 & 31 & 36 \\
39 & 31 & 36 \\
39 & 31 & 36 \\
39 & 33 & 33 \\
39 & 33 & 31\end{array}$ & $\begin{array}{l}113 \\
113 \\
113 \\
113 \\
113\end{array}$ & $\begin{array}{ll}0 & 32 \\
0 & 32 \\
0 & 32 \\
1 & 28 \\
1 & 28\end{array}$ & $\begin{array}{r}<.05 \\
.10 \\
.50 \\
5.00 \\
2.00\end{array}$ & $\begin{array}{l}.02 \\
<.02 \\
<.02 \\
.10 \\
.05\end{array}$ & $\begin{array}{l}<.05 \\
<.05 \\
<.05 \\
.20 \\
.20\end{array}$ & $\begin{array}{l}.300 \\
.300 \\
.500 \\
.150 \\
.100\end{array}$ & $\begin{array}{r}10 \\
<10 \\
<10 \\
20 \\
100\end{array}$ & $\begin{array}{l}<.50 \\
<.50 \\
N \\
N \\
N .50\end{array}$ & $\begin{array}{l}N \\
N \\
N \\
N \\
N\end{array}$ & $\begin{array}{l}\mathrm{N} \\
\mathrm{N} \\
\mathrm{N} \\
\mathrm{N} \\
\mathrm{N}\end{array}$ \\
\hline $\begin{array}{l}70 H 3074 A \\
70 A 3074 B \\
70 H 3074 C \\
70 H 3074 D \\
70 H 3075\end{array}$ & $\begin{array}{lll}39 & 33 & 34 \\
39 & 33 & 34 \\
39 & 33 & 34 \\
39 & 33 & 34 \\
39 & 33 & 35\end{array}$ & $\begin{array}{l}113 \\
113 \\
113 \\
113 \\
113\end{array}$ & $\begin{array}{ll}1 & 37 \\
1 & 37 \\
1 & 37 \\
1 & 37 \\
1 & 55\end{array}$ & $\begin{array}{r}5.00 \\
.50 \\
.10 \\
.10 \\
.07\end{array}$ & $\begin{array}{r}.50 \\
.30 \\
.02 \\
.50 \\
3.00\end{array}$ & $\begin{array}{r}.10 \\
.10 \\
.20 \\
20.00 \\
>20.00\end{array}$ & $\begin{array}{l}.200 \\
.500 \\
.500 \\
.005 \\
.002\end{array}$ & $\begin{array}{r}30 \\
20 \\
100 \\
500 \\
500\end{array}$ & $\begin{array}{l}N \\
N \\
N \\
N \\
N\end{array}$ & $\begin{array}{l}N \\
N \\
N \\
N \\
N\end{array}$ & $\begin{array}{l}N \\
N \\
N \\
N \\
N\end{array}$ \\
\hline $\begin{array}{l}70 H 3076 \\
70 H 3079 \\
70 H 3080 A \\
70 H 3080 B \\
70 H 3082 A\end{array}$ & $\begin{array}{lll}39 & 33 & 32 \\
39 & 33 & 37 \\
39 & 33 & 33 \\
39 & 33 & 33 \\
39 & 33 & 49\end{array}$ & $\begin{array}{l}113 \\
113 \\
113 \\
113 \\
113\end{array}$ & $\begin{array}{rr}2 & 8 \\
2 & 10 \\
1 & 47 \\
1 & 47 \\
2 & 28\end{array}$ & $\begin{array}{r}2.00 \\
.15 \\
5.00 \\
2.00 \\
3.00\end{array}$ & $\begin{array}{r}.05 \\
10.00 \\
3.00 \\
2.00 \\
1.00\end{array}$ & $\begin{array}{r}.50 \\
20.00 \\
2.00 \\
1.00 \\
.20\end{array}$ & $\begin{array}{l}.050 \\
.002 \\
.300 \\
.500 \\
.500\end{array}$ & $\begin{array}{r}200 \\
300 \\
1,000 \\
500 \\
100\end{array}$ & $\begin{array}{l}7.00 \\
N \\
<.50 \\
3.00 \\
10.00\end{array}$ & $\begin{array}{r}N \\
N \\
N \\
N \\
500\end{array}$ & $\begin{array}{l}N \\
N \\
N \\
N \\
N\end{array}$ \\
\hline $\begin{array}{l}70 H 3082 B \\
70 H 3082 C \\
70 H 3083 A \\
70 H 3083 B \\
70 H 3083 C\end{array}$ & $\begin{array}{lll}39 & 33 & 49 \\
39 & 33 & 49 \\
39 & 32 & 40 \\
39 & 32 & 40 \\
39 & 32 & 39\end{array}$ & $\begin{array}{l}113 \\
113 \\
113 \\
113 \\
113\end{array}$ & $\begin{array}{ll}2 & 28 \\
2 & 28 \\
1 & 44 \\
1 & 44 \\
1 & 43\end{array}$ & $\begin{array}{r}.05 \\
.50 \\
.50 \\
1.00 \\
.05\end{array}$ & $\begin{array}{r}3.00 \\
.10 \\
1.00 \\
.02 \\
.05\end{array}$ & $\begin{array}{r}>20.00 \\
15.00 \\
>20.00 \\
.10 \\
.07\end{array}$ & $\begin{array}{l}.002 \\
.100 \\
.010 \\
.100 \\
.100\end{array}$ & $\begin{array}{r}300 \\
200 \\
300 \\
20 \\
<10\end{array}$ & $\begin{array}{l}5.00 \\
N \\
1.00 \\
2.00 \\
N\end{array}$ & $\begin{array}{l}N \\
N \\
N \\
N \\
N\end{array}$ & $\begin{array}{l}N \\
N \\
N \\
N \\
N\end{array}$ \\
\hline $\begin{array}{l}70 H 3084 \\
70 H 3085 A \\
70 H 3085 B \\
70 H 3086 A \\
70 H 3086 B\end{array}$ & $\begin{array}{lll}39 & 32 & 49 \\
39 & 33 & 11 \\
39 & 33 & 11 \\
39 & 32 & 59 \\
39 & 32 & 59\end{array}$ & $\begin{array}{l}113 \\
113 \\
113 \\
113 \\
113\end{array}$ & $\begin{array}{ll}1 & 28 \\
1 & 58 \\
1 & 58 \\
0 & 53 \\
0 & 53\end{array}$ & $\begin{array}{l}3.00 \\
2.00 \\
5.00 \\
1.00 \\
3.00\end{array}$ & $\begin{array}{r}.02 \\
.02 \\
1.50 \\
.05 \\
1.00\end{array}$ & $\begin{array}{r}.10 \\
.10 \\
10.00 \\
.10 \\
.10\end{array}$ & $\begin{array}{l}.150 \\
.050 \\
.150 \\
.100 \\
.200\end{array}$ & $\begin{array}{r}20 \\
150 \\
500 \\
<10 \\
100\end{array}$ & $\begin{array}{c}2.00 \\
20.00 \\
N \\
N \\
N\end{array}$ & $\begin{array}{r}N \\
200 \\
N \\
N \\
N\end{array}$ & $\begin{array}{l}N \\
N \\
N \\
N \\
N\end{array}$ \\
\hline $\begin{array}{l}70 H 3086 C \\
70 H 3087 \\
70 H 3088 A \\
7 D H 3088 B \\
70 H 3089 A\end{array}$ & $\begin{array}{rrr}39 & 32 & 59 \\
39 & 32 & 56 \\
39 & 32 & 54 \\
39 & 32 & 54 \\
39 & 34 & 4\end{array}$ & $\begin{array}{l}113 \\
113 \\
113 \\
113 \\
113\end{array}$ & $\begin{array}{ll}0 & 53 \\
0 & 52 \\
0 & 45 \\
0 & 45 \\
1 & 53\end{array}$ & $\begin{array}{r}10.00 \\
.50 \\
2.00 \\
2.00 \\
.15\end{array}$ & $\begin{array}{l}.50 \\
.02 \\
.50 \\
.70 \\
.50\end{array}$ & $\begin{array}{r}2.00 \\
.20 \\
.10 \\
20.00 \\
.10\end{array}$ & $\begin{array}{l}.020 \\
.100 \\
.300 \\
.005 \\
.150\end{array}$ & $\begin{array}{r}>5,000 \\
500 \\
100 \\
1,500 \\
1,000\end{array}$ & $\begin{array}{l}\text { N } \\
N \\
N \\
N \\
N\end{array}$ & $\begin{array}{l}N \\
N \\
N \\
N \\
N\end{array}$ & $\begin{array}{l}N \\
N \\
N \\
N \\
N\end{array}$ \\
\hline $\begin{array}{l}\text { 7DH3089B } \\
7 D H 3127 \\
\text { 7DH3128 } \\
\text { 7DH3185A } \\
7 D H 3185 B\end{array}$ & $\begin{array}{rrr}39 & 34 & 4 \\
39 & 31 & 27 \\
39 & 31 & 42 \\
39 & 31 & 35 \\
39 & 31 & 35\end{array}$ & $\begin{array}{l}113 \\
113 \\
113 \\
113 \\
113\end{array}$ & $\begin{array}{rr}1 & 53 \\
0 & 26 \\
0 & 4 \\
0 & 33 \\
0 & 33\end{array}$ & $\begin{array}{r}2.00 \\
.10 \\
2.00 \\
3.00 \\
2.00\end{array}$ & $\begin{array}{r}.20 \\
.05 \\
1.00 \\
.20 \\
.30\end{array}$ & $\begin{array}{r}.20 \\
<.05 \\
1.50 \\
.07 \\
.20\end{array}$ & $\begin{array}{l}.200 \\
.150 \\
.200 \\
.200 \\
.150\end{array}$ & $\begin{array}{r}500 \\
10 \\
700 \\
<10 \\
300\end{array}$ & $\begin{array}{l}\mathbf{N} \\
N \\
N \\
N \\
N\end{array}$ & $\begin{array}{r}300 \\
<200 \\
N \\
N \\
N\end{array}$ & $\begin{array}{l}N \\
N \\
N \\
N \\
N\end{array}$ \\
\hline $\begin{array}{l}\text { 7DH3186 } \\
\text { 7DH3188A } \\
\text { 7DH31888 } \\
\text { 7DH3188C } \\
7 D H 3190\end{array}$ & $\begin{array}{lll}39 & 31 & 35 \\
39 & 31 & 35 \\
39 & 31 & 35 \\
39 & 31 & 35 \\
39 & 31 & 35\end{array}$ & $\begin{array}{l}113 \\
113 \\
113 \\
113 \\
113\end{array}$ & $\begin{array}{ll}0 & 33 \\
0 & 33 \\
0 & 33 \\
0 & 33 \\
0 & 33\end{array}$ & $\begin{array}{r}3.00 \\
2.00 \\
1.00 \\
.10 \\
1.00\end{array}$ & $\begin{array}{r}.30 \\
.70 \\
3.00 \\
.70 \\
1.50\end{array}$ & $\begin{array}{r}.10 \\
20.00 \\
20.00 \\
20.00 \\
>20.00\end{array}$ & $\begin{array}{l}.200 \\
.020 \\
.020 \\
.005 \\
.070\end{array}$ & $\begin{array}{r}10 \\
150 \\
500 \\
50 \\
500\end{array}$ & $\begin{array}{l}N \\
N \\
N \\
N \\
N\end{array}$ & $\begin{array}{l}N \\
N \\
N \\
N \\
N\end{array}$ & $\begin{array}{l}N \\
N \\
N \\
N \\
N\end{array}$ \\
\hline $\begin{array}{l}\text { 7DH3191A } \\
\text { 7DH3191B } \\
\text { 7DH3192 } \\
\text { 7DH3193 } \\
7 D H 3194\end{array}$ & $\begin{array}{lll}39 & 31 & 35 \\
39 & 31 & 35 \\
39 & 31 & 35 \\
39 & 31 & 35 \\
39 & 31 & 35\end{array}$ & $\begin{array}{l}113 \\
113 \\
113 \\
113 \\
113\end{array}$ & $\begin{array}{ll}0 & 33 \\
0 & 33 \\
0 & 33 \\
0 & 33 \\
0 & 33\end{array}$ & $\begin{array}{r}2.00 \\
1.00 \\
2.00 \\
.50 \\
5.00\end{array}$ & $\begin{array}{r}.70 \\
1.00 \\
.10 \\
1.00 \\
1.00\end{array}$ & $\begin{array}{r}>20.00 \\
>20.00 \\
10.00 \\
>20.00 \\
.20\end{array}$ & $\begin{array}{l}.050 \\
.100 \\
.100 \\
.020 \\
.200\end{array}$ & $\begin{array}{r}1,000 \\
500 \\
700 \\
300 \\
500\end{array}$ & $\begin{array}{l}N \\
N \\
N \\
N \\
N\end{array}$ & $\begin{array}{l}N \\
N \\
N \\
N \\
N\end{array}$ & $\begin{array}{l}N \\
N \\
N \\
N \\
N\end{array}$ \\
\hline
\end{tabular}


TABLE 4--RESULTS OF ANALYSES, DETROIT MINING DISTRICT, UTAH--Continued

\begin{tabular}{|c|c|c|c|c|c|c|c|c|c|c|c|c|c|}
\hline Sample & $\begin{array}{c}\text { B-ppm } \\
\text { s }\end{array}$ & $\begin{array}{c}\text { Ba-ppm } \\
\mathrm{s}\end{array}$ & $\begin{array}{c}\text { Be-ppm } \\
s\end{array}$ & $\begin{array}{c}\text { Bi-ppm } \\
s\end{array}$ & $\begin{array}{l}\text { Cd-ppm } \\
s\end{array}$ & $\begin{array}{l}\text { Co-ppm } \\
\mathrm{s}\end{array}$ & $\begin{array}{c}\text { Cr-ppm } \\
\mathrm{s}\end{array}$ & $\begin{array}{c}\text { Cu-ppm } \\
s\end{array}$ & $\begin{array}{l}\text { La-ppm } \\
s\end{array}$ & $\begin{array}{l}\text { Mo-ppm } \\
\mathrm{s}\end{array}$ & $\begin{array}{l}\text { Nb-ppm } \\
\text { s }\end{array}$ & $\begin{array}{c}\text { Ni-ppm } \\
\text { s }\end{array}$ & $\begin{array}{l}\text { Pb-ppm } \\
\text { s }\end{array}$ \\
\hline $\begin{array}{l}8 D H 3043 A \\
8 D H 3043 B \\
8 D H 3043 C \\
7 D H 3073 A \\
7 D H 3073 B\end{array}$ & $\begin{array}{l}15 \\
20 \\
15 \\
10 \\
10\end{array}$ & $\begin{array}{r}100 \\
300 \\
300 \\
1,000 \\
300\end{array}$ & $\begin{array}{c}N \\
N \\
N \\
1.0 \\
<1.0\end{array}$ & $\begin{array}{l}N \\
N \\
N \\
N \\
N\end{array}$ & $\begin{array}{l}N \\
N \\
N \\
N \\
N\end{array}$ & $\begin{array}{r}N \\
N \\
N \\
10 \\
<5\end{array}$ & $\begin{array}{l}70 \\
30 \\
50 \\
30 \\
10\end{array}$ & $\begin{array}{r}5 \\
5 \\
5 \\
10 \\
20\end{array}$ & $\begin{array}{r}<50 \\
50 \\
<50 \\
50 \\
<20\end{array}$ & $\begin{array}{l}N \\
N \\
N \\
N \\
5\end{array}$ & $\begin{array}{l}N \\
N \\
N \\
N \\
N\end{array}$ & $\begin{array}{r}<5 \\
<5 \\
<5 \\
5 \\
5\end{array}$ & $\begin{array}{r}200 \\
150 \\
100 \\
20 \\
50\end{array}$ \\
\hline $\begin{array}{l}\text { TOH3074A } \\
70 A 3074 B \\
70 H 3074 C \\
70 H 30740 \\
7 D H 3075\end{array}$ & $\begin{array}{r}200 \\
100 \\
10 \\
N \\
N\end{array}$ & $\begin{array}{r}200 \\
300 \\
50 \\
N \\
<20\end{array}$ & $\begin{array}{r}5.0 \\
3.0 \\
<1.0 \\
<1.0 \\
<1.0\end{array}$ & $\begin{array}{l}N \\
N \\
N \\
N \\
N\end{array}$ & $\begin{array}{l}N \\
N \\
N \\
N \\
N\end{array}$ & $\begin{array}{l}<5 \\
N \\
N \\
N \\
N\end{array}$ & $\begin{array}{r}70 \\
70 \\
50 \\
N \\
N\end{array}$ & $\begin{array}{r}10 \\
5 \\
5 \\
<5 \\
<5\end{array}$ & $\begin{array}{r}100 \\
150 \\
50 \\
<20 \\
<20\end{array}$ & $\begin{array}{l}N \\
N \\
N \\
N \\
N\end{array}$ & $\begin{array}{r}<20 \\
20 \\
<20 \\
N \\
N\end{array}$ & $\begin{array}{r}7 \\
7 \\
5 \\
<5 \\
<5\end{array}$ & $\begin{array}{r}20 \\
100 \\
<10 \\
<10 \\
20\end{array}$ \\
\hline $\begin{array}{l}\text { 70H3076 } \\
70 H 3079 \\
70 H 3080 A \\
70 H 3080 B \\
70 H 3082 A\end{array}$ & $\begin{array}{r}15 \\
N \\
10 \\
20 \\
15\end{array}$ & $\begin{array}{l}150 \\
<20 \\
500 \\
700 \\
150\end{array}$ & $\begin{array}{r}<1.0 \\
<1.0 \\
2.0 \\
2.0 \\
3.0\end{array}$ & $\begin{array}{r}N \\
N \\
N \\
N \\
<10\end{array}$ & $\begin{array}{l}N \\
N \\
N \\
N \\
N\end{array}$ & $\begin{array}{r}N \\
N \\
50 \\
15 \\
N\end{array}$ & $\begin{array}{r}<10 \\
N \\
20 \\
20 \\
50\end{array}$ & $\begin{array}{r}20 \\
<5 \\
50 \\
2,000 \\
2,000\end{array}$ & $\begin{array}{r}N \\
N \\
<20 \\
<20 \\
70\end{array}$ & $\begin{array}{l}\mathbf{N} \\
\mathbf{N} \\
\mathbf{N} \\
\mathbf{N} \\
7\end{array}$ & $\begin{array}{l}N \\
N \\
N \\
N \\
N\end{array}$ & $\begin{array}{r}<5 \\
<5 \\
20 \\
70 \\
5\end{array}$ & $\begin{array}{r}70 \\
10 \\
70 \\
70 \\
100\end{array}$ \\
\hline $\begin{array}{l}70 H 3082 B \\
7 D H 3082 C \\
7 D H 3083 A \\
7 D H 3083 B \\
7 D H 3083 C\end{array}$ & $\begin{array}{r}N \\
10 \\
N \\
10 \\
10\end{array}$ & $\begin{array}{l}<20 \\
100 \\
<20 \\
<20 \\
<20\end{array}$ & $\begin{array}{r}<1.0 \\
<1.0 \\
<1.0 \\
<1.0 \\
1.0\end{array}$ & $\begin{array}{r}N \\
200 \\
N \\
<10 \\
N\end{array}$ & $\begin{array}{l}N \\
N \\
N \\
N \\
N\end{array}$ & $\begin{array}{l}N \\
<5 \\
<5 \\
<5 \\
<5\end{array}$ & $\begin{array}{r}N \\
<10 \\
<10 \\
<10 \\
<10\end{array}$ & $\begin{array}{l}50 \\
15 \\
15 \\
70 \\
50\end{array}$ & $\begin{array}{r}N \\
N \\
N \\
N \\
<20\end{array}$ & $\begin{array}{r}N \\
N \\
N \\
<5 \\
N\end{array}$ & $\begin{array}{l}N \\
N \\
N \\
N \\
N\end{array}$ & $\begin{array}{r}<5 \\
7 \\
5 \\
5 \\
20\end{array}$ & $\begin{array}{r}<10 \\
20 \\
30 \\
20 \\
20\end{array}$ \\
\hline $\begin{array}{l}70 H 3084 \\
70 H 3085 A \\
70 H 3085 B \\
7 D H 3086 A \\
70 H 3086 B\end{array}$ & $\begin{array}{r}10 \\
10 \\
<10 \\
10 \\
10\end{array}$ & $\begin{array}{r}50 \\
<20 \\
150 \\
500 \\
200\end{array}$ & $\begin{array}{c}N \\
N \\
<1.0 \\
1.0 \\
1.0\end{array}$ & $\begin{array}{r}N \\
300 \\
N \\
N \\
N\end{array}$ & $\begin{array}{l}N \\
N \\
N \\
N \\
N\end{array}$ & $\begin{array}{r}<5 \\
N \\
10 \\
N \\
20\end{array}$ & $\begin{array}{r}<10 \\
<10 \\
20 \\
10 \\
<10\end{array}$ & $\begin{array}{r}15 \\
300 \\
20 \\
<5 \\
20\end{array}$ & $\begin{array}{r}N \\
N \\
50 \\
50 \\
50\end{array}$ & $\begin{array}{l}<5 \\
N \\
N \\
N \\
N\end{array}$ & $\begin{array}{l}\mathbf{N} \\
\mathbf{N} \\
\mathbf{N} \\
\mathbf{N} \\
\mathbf{N}\end{array}$ & $\begin{array}{r}10 \\
5 \\
50 \\
<5 \\
10\end{array}$ & $\begin{array}{l}50 \\
30 \\
50 \\
70 \\
20\end{array}$ \\
\hline $\begin{array}{l}\text { 7DH3086C } \\
7 D H 3087 \\
7 D H 3088 A \\
70 H 3088 B \\
7 D H 3089 A\end{array}$ & $\begin{array}{r}<10 \\
15 \\
50 \\
N \\
10\end{array}$ & $\begin{array}{l}150 \\
100 \\
500 \\
100 \\
300\end{array}$ & $\begin{array}{r}15.0 \\
<1.0 \\
3.0 \\
<1.0 \\
<1.0\end{array}$ & $\begin{array}{l}N \\
N \\
N \\
N \\
N\end{array}$ & $\begin{array}{l}N \\
N \\
N \\
N \\
N\end{array}$ & $\begin{array}{r}200 \\
<5 \\
10 \\
N \\
<5\end{array}$ & $\begin{array}{r}N \\
10 \\
30 \\
N \\
10\end{array}$ & $\begin{array}{r}7 \\
5 \\
10 \\
5 \\
10\end{array}$ & $\begin{array}{r}N \\
N \\
50 \\
N \\
<20\end{array}$ & $\begin{array}{r}N \\
10 \\
N \\
5 \\
N\end{array}$ & $\begin{array}{r}N \\
N \\
<20 \\
N \\
N\end{array}$ & $\begin{array}{r}150 \\
<5 \\
20 \\
<5 \\
<5\end{array}$ & $\begin{array}{l}20 \\
30 \\
50 \\
10 \\
70\end{array}$ \\
\hline $\begin{array}{l}70 H 3089 B \\
70 H 3127 \\
70 H 3128 \\
70 H 3185 A \\
70 H 3185 B\end{array}$ & $\begin{array}{l}20 \\
10 \\
50 \\
50 \\
20\end{array}$ & $\begin{array}{l}500 \\
200 \\
700 \\
200 \\
100\end{array}$ & $\begin{array}{l}2.0 \\
N \\
1.5 \\
1.0 \\
2.0\end{array}$ & $\begin{array}{l}N \\
N \\
N \\
N \\
N\end{array}$ & $\begin{array}{l}N \\
N \\
N \\
N \\
N\end{array}$ & $\begin{array}{r}<5 \\
N \\
10 \\
50 \\
50\end{array}$ & $\begin{array}{r}10 \\
10 \\
<10 \\
50 \\
<10\end{array}$ & $\begin{array}{r}7 \\
10 \\
10 \\
100 \\
15\end{array}$ & $\begin{array}{r}100 \\
N \\
100 \\
70 \\
<20\end{array}$ & $\begin{array}{l}N \\
N \\
N \\
N \\
7\end{array}$ & $\begin{array}{r}<20 \\
N \\
N \\
<20 \\
<20\end{array}$ & $\begin{array}{l}5 \\
<5 \\
<5 \\
70 \\
70\end{array}$ & $\begin{array}{r}20 \\
50 \\
100 \\
70 \\
20\end{array}$ \\
\hline $\begin{array}{l}70 H 3186 \\
70 H 3188 A \\
70 H 3188 B \\
70 H 3188 C \\
70 H 3190\end{array}$ & $\begin{array}{r}30 \\
N \\
N \\
N \\
<10\end{array}$ & $\begin{array}{r}1,000 \\
<20 \\
300 \\
<20 \\
100\end{array}$ & $\begin{array}{c}2.0 \\
N \\
N \\
N \\
<1.0\end{array}$ & $\begin{array}{l}N \\
N \\
N \\
N \\
N\end{array}$ & $\begin{array}{l}\mathrm{N} \\
\mathrm{N} \\
\mathrm{N} \\
\mathrm{N} \\
\mathrm{N}\end{array}$ & $\begin{array}{r}30 \\
50 \\
10 \\
N \\
N\end{array}$ & $\begin{array}{r}<10 \\
N \\
N \\
<10 \\
<10\end{array}$ & $\begin{array}{l}15 \\
10 \\
15 \\
<5 \\
<5\end{array}$ & $\begin{array}{r}100 \\
N \\
<20 \\
N \\
<20\end{array}$ & $\begin{array}{l}\text { N } \\
7 \\
N \\
N \\
N\end{array}$ & $\begin{array}{l}N \\
N \\
N \\
N \\
N\end{array}$ & $\begin{array}{l}10 \\
50 \\
10 \\
<5 \\
<5\end{array}$ & $\begin{array}{r}50 \\
<10 \\
50 \\
N \\
100\end{array}$ \\
\hline $\begin{array}{l}\text { 70H3191A } \\
70 H 3191 B \\
70 H 3192 \\
70 H 3193 \\
70 H 3194\end{array}$ & $\begin{array}{r}N \\
<10 \\
10 \\
N \\
50\end{array}$ & $\begin{array}{r}50 \\
70 \\
100 \\
20 \\
300\end{array}$ & $\begin{array}{c}<1.0 \\
<1.0 \\
<1.0 \\
N \\
2.0\end{array}$ & $\begin{array}{r}N \\
N \\
10 \\
N \\
N\end{array}$ & $\begin{array}{l}N \\
N \\
N \\
N \\
N\end{array}$ & $\begin{array}{r}10 \\
N \\
20 \\
N \\
30\end{array}$ & $\begin{array}{r}<10 \\
<10 \\
<10 \\
<10 \\
50\end{array}$ & $\begin{array}{r}<5 \\
<5 \\
20 \\
5 \\
15\end{array}$ & $\begin{array}{r}N \\
<20 \\
<20 \\
N \\
70\end{array}$ & $\begin{array}{r}N \\
N \\
<5 \\
N \\
N\end{array}$ & $\begin{array}{r}N \\
N \\
N \\
N \\
N \\
<20\end{array}$ & $\begin{array}{r}15 \\
5 \\
10 \\
<5 \\
70\end{array}$ & $\begin{array}{r}150 \\
10 \\
50 \\
20 \\
<10\end{array}$ \\
\hline
\end{tabular}


TABLE 4--RESULTS OF ANALYSES, DETROIT MINING DISTRICT, UTAH--Cont inuEd

\begin{tabular}{|c|c|c|c|c|c|c|c|c|c|c|c|c|}
\hline Sample & $\begin{array}{l}\text { Sb-ppm } \\
\mathbf{s}\end{array}$ & $\begin{array}{c}\text { Sc-ppm } \\
\text { s }\end{array}$ & $\begin{array}{l}\text { Sn-ppm } \\
s\end{array}$ & $\begin{array}{c}\text { Sr-ppm } \\
\text { s }\end{array}$ & $\begin{array}{c}\text { V-ppm } \\
\text { s }\end{array}$ & $\begin{array}{c}W-p p m \\
\text { s }\end{array}$ & $\begin{array}{c}\text { Y-ppm } \\
\text { s }\end{array}$ & $\begin{array}{c}\text { Zn-ppm } \\
\text { s }\end{array}$ & $\begin{array}{c}2 r-p p m \\
s\end{array}$ & $\begin{array}{c}\text { Th-ppm } \\
\mathbf{s}\end{array}$ & $\begin{array}{c}\text { Au-ppm } \\
\text { aa }\end{array}$ & $\begin{array}{l}\text { AS-PPM } \\
\text { ICP }\end{array}$ \\
\hline $\begin{array}{l}80 H 3043 A \\
80 H 3043 B \\
80 H 3043 C \\
70 H 3073 A \\
70 H 3073 B\end{array}$ & $\begin{array}{l}N \\
N \\
N \\
N \\
N\end{array}$ & $\begin{array}{r}N \\
N \\
N \\
20 \\
5\end{array}$ & $\begin{array}{l}N \\
N \\
N \\
N \\
N\end{array}$ & $\begin{array}{l}100 \\
150 \\
200 \\
300 \\
200\end{array}$ & $\begin{array}{l}30 \\
50 \\
50 \\
70 \\
20\end{array}$ & $\begin{array}{l}N \\
N \\
N \\
N \\
N\end{array}$ & $\begin{array}{r}N \\
N \\
N \\
100 \\
N\end{array}$ & $\begin{array}{r}N \\
N \\
N \\
<200 \\
<200\end{array}$ & $\begin{array}{r}20 \\
70 \\
150 \\
700 \\
150\end{array}$ & $\begin{array}{l}\mathbf{N} \\
\mathbf{N} \\
\mathbf{N} \\
\mathbf{N} \\
\mathbf{N}\end{array}$ & $\begin{array}{l}.20 \\
.40 \\
.30 \\
\mathrm{~N} \\
.05\end{array}$ & $\begin{array}{c}<5.0 \\
<5.0 \\
13.0 \\
N \\
N\end{array}$ \\
\hline $\begin{array}{l}70 H 3074 A \\
70 A 3074 B \\
7 D H 3074 C \\
7 D H 3074 D \\
7 D H 3075\end{array}$ & $\begin{array}{l}N \\
N \\
N \\
N \\
N\end{array}$ & $\begin{array}{r}15 \\
20 \\
<5 \\
N \\
N\end{array}$ & $\begin{array}{r}N \\
15 \\
N \\
N \\
N\end{array}$ & $\begin{array}{r}300 \\
<100 \\
N \\
500 \\
500\end{array}$ & $\begin{array}{r}100 \\
100 \\
20 \\
10 \\
10\end{array}$ & $\begin{array}{l}N \\
N \\
N \\
N \\
N\end{array}$ & $\begin{array}{r}50 \\
50 \\
<10 \\
N \\
N\end{array}$ & $\begin{array}{l}<200 \\
<200 \\
<200 \\
<200 \\
<200\end{array}$ & $\begin{array}{l}100 \\
100 \\
150 \\
<10 \\
<10\end{array}$ & $\begin{array}{l}\mathbf{N} \\
\mathbf{N} \\
\mathbf{N} \\
\mathbf{N} \\
\mathbf{N}\end{array}$ & $\begin{array}{l}N \\
.35 \\
.20 \\
N \\
N\end{array}$ & $\begin{array}{c}30.0 \\
N \\
N \\
N \\
N\end{array}$ \\
\hline $\begin{array}{l}\text { 7DH3076 } \\
\text { 7DH3079 } \\
\text { 7DH3080A } \\
\text { 7DH3080B } \\
70 H 3082 A\end{array}$ & $\begin{array}{r}N \\
N \\
N \\
N \\
300\end{array}$ & $\begin{array}{r}<5 \\
N \\
20 \\
15 \\
10\end{array}$ & $\begin{array}{c}N \\
N \\
N \\
N \\
15\end{array}$ & $\begin{array}{r}<100 \\
N \\
500 \\
200 \\
300\end{array}$ & $\begin{array}{r}15 \\
10 \\
200 \\
100 \\
150\end{array}$ & $\begin{array}{l}\mathbf{N} \\
\mathbf{N} \\
\mathbf{N} \\
\mathbf{N} \\
\mathbf{N}\end{array}$ & $\begin{array}{r}20 \\
<10 \\
20 \\
20 \\
20\end{array}$ & $\begin{array}{r}<200 \\
<200 \\
<200 \\
700 \\
<200\end{array}$ & $\begin{array}{r}<10 \\
N \\
150 \\
150 \\
150\end{array}$ & $\begin{array}{l}N \\
N \\
N \\
N \\
N\end{array}$ & $\begin{array}{l}.85 \\
\mathrm{~N} \\
\mathrm{~N} \\
\mathrm{~N} \\
.75\end{array}$ & $\begin{array}{c}40.0 \\
N \\
N \\
20.0 \\
500.0\end{array}$ \\
\hline $\begin{array}{l}70 H 3082 B \\
70 H 3082 C \\
70 H 3083 A \\
70 H 3083 B \\
7 D H 3083 C\end{array}$ & $\begin{array}{l}N \\
N \\
N \\
N \\
N\end{array}$ & $\begin{array}{r}N \\
<5 \\
N \\
N \\
<5\end{array}$ & $\begin{array}{r}N \\
N \\
N \\
20 \\
N\end{array}$ & $\begin{array}{r}500 \\
N \\
700 \\
N \\
300\end{array}$ & $\begin{array}{l}10 \\
15 \\
15 \\
20 \\
50\end{array}$ & $\begin{array}{l}\mathbf{N} \\
\mathbf{N} \\
\mathbf{N} \\
\mathbf{N} \\
\mathbf{N}\end{array}$ & $\begin{array}{r}<10 \\
30 \\
<10 \\
N \\
<10\end{array}$ & $\begin{array}{r}N \\
<200 \\
<200 \\
<200 \\
<200\end{array}$ & $\begin{array}{r}<10 \\
50 \\
<10 \\
50 \\
70\end{array}$ & $\begin{array}{l}\text { N } \\
N \\
N \\
N \\
N\end{array}$ & $\begin{array}{l}\dot{N}^{N} \\
2^{2.60} \\
2.10\end{array}$ & $\begin{array}{c}N \\
N \\
10.0 \\
30.0 \\
N\end{array}$ \\
\hline $\begin{array}{l}70 H 3084 \\
70 H 3085 A \\
70 H 3085 B \\
7 D H 3086 A \\
7 D H 3086 B\end{array}$ & $\begin{array}{r}N \\
1,500 \\
N \\
N \\
N\end{array}$ & $\begin{array}{r}<5 \\
<5 \\
15 \\
5 \\
10\end{array}$ & $\begin{array}{r}N \\
100 \\
N \\
N \\
N\end{array}$ & $\begin{array}{r}N \\
N \\
300 \\
500 \\
N\end{array}$ & $\begin{array}{r}20 \\
10 \\
100 \\
50 \\
100\end{array}$ & $\begin{array}{l}N \\
N \\
N \\
N \\
N\end{array}$ & $\begin{array}{r}N \\
N \\
20 \\
50 \\
20\end{array}$ & $\begin{array}{r}<200 \\
<200 \\
N \\
<200 \\
N\end{array}$ & $\begin{array}{r}50 \\
30 \\
70 \\
200 \\
100\end{array}$ & $\begin{array}{l}N \\
N \\
N \\
N \\
N\end{array}$ & $\begin{array}{l}.10 \\
1.50 \\
N \\
N \\
N\end{array}$ & $\begin{array}{c}60.0 \\
500.0 \\
30.0 \\
N \\
20.0\end{array}$ \\
\hline $\begin{array}{l}70 H 3086 C \\
7 D H 3087 \\
70 H 3088 A \\
70 H 3088 B \\
70 H 3089 A\end{array}$ & $\begin{array}{l}N \\
N \\
N \\
N \\
N\end{array}$ & $\begin{array}{l}<5 \\
<5 \\
15 \\
N \\
5\end{array}$ & $\begin{array}{r}N \\
10 \\
N \\
N \\
N\end{array}$ & $\begin{array}{r}N \\
N \\
N \\
500 \\
N\end{array}$ & $\begin{array}{r}<10 \\
30 \\
100 \\
10 \\
70\end{array}$ & $\begin{array}{l}N \\
N \\
N \\
N \\
N\end{array}$ & $\begin{array}{r}30 \\
N \\
50 \\
15 \\
10\end{array}$ & $\begin{array}{r}1,000 \\
<200 \\
<200 \\
<200 \\
N\end{array}$ & $\begin{array}{l}<10 \\
100 \\
150 \\
100 \\
100\end{array}$ & $\begin{array}{l}\text { N } \\
N \\
N \\
N \\
N\end{array}$ & $\begin{array}{l}N \\
N \\
N \\
N \\
N\end{array}$ & $\begin{array}{c}20.0 \\
N \\
20.0 \\
130.0 \\
600.0\end{array}$ \\
\hline $\begin{array}{l}70 H 3089 B \\
70 H 3127 \\
70 H 3128 \\
70 H 3185 A \\
7 D H 3185 B\end{array}$ & $\begin{array}{l}N \\
N \\
N \\
N \\
N\end{array}$ & $\begin{array}{r}10 \\
<5 \\
15 \\
20 \\
5\end{array}$ & $\begin{array}{r}N \\
N \\
N \\
10 \\
N\end{array}$ & $\begin{array}{r}N \\
<100 \\
<100 \\
300 \\
N\end{array}$ & $\begin{array}{r}50 \\
70 \\
100 \\
100 \\
70\end{array}$ & $\begin{array}{l}\mathbf{N} \\
\mathbf{N} \\
\mathbf{N} \\
\mathbf{N} \\
\mathbf{N}\end{array}$ & $\begin{array}{r}50 \\
N \\
20 \\
30 \\
20\end{array}$ & $\begin{array}{r}N \\
<200 \\
<200 \\
<200 \\
N\end{array}$ & $\begin{array}{r}300 \\
150 \\
150 \\
50 \\
150\end{array}$ & $\begin{array}{l}N \\
N \\
N \\
N \\
N\end{array}$ & $\begin{array}{l}N \\
.65 \\
N \\
N \\
N\end{array}$ & $\begin{array}{c}300.0 \\
N \\
N \\
N \\
N\end{array}$ \\
\hline $\begin{array}{l}\text { 7DH3186 } \\
\text { 70H3188A } \\
\text { 70H3188B } \\
\text { 7DH3188C } \\
7 D H 3190\end{array}$ & $\begin{array}{l}N \\
N \\
N \\
N \\
N\end{array}$ & $\begin{array}{r}15 \\
<5 \\
<5 \\
N \\
10\end{array}$ & $\begin{array}{l}\mathbf{N} \\
N \\
N \\
N \\
N\end{array}$ & $\begin{array}{r}500 \\
500 \\
500 \\
500 \\
1,000\end{array}$ & $\begin{array}{r}150 \\
50 \\
20 \\
10 \\
20\end{array}$ & $\begin{array}{l}\mathbf{N} \\
\mathbf{N} \\
\mathbf{N} \\
\mathbf{N} \\
\mathbf{N}\end{array}$ & $\begin{array}{r}20 \\
<10 \\
20 \\
N \\
20\end{array}$ & $\begin{array}{r}<200 \\
N \\
N \\
N \\
N\end{array}$ & $\begin{array}{r}200 \\
<10 \\
20 \\
<10 \\
30\end{array}$ & $\begin{array}{l}N \\
N \\
N \\
N \\
N\end{array}$ & $\begin{array}{l}.10 \\
N \\
N \\
N \\
N\end{array}$ & $\begin{array}{c}20.0 \\
10.0 \\
N \\
N \\
N\end{array}$ \\
\hline $\begin{array}{l}\text { 7DH3191A } \\
\text { 7DH3191B } \\
\text { 7DH3192 } \\
\text { 7DH3193 } \\
70 H 3194\end{array}$ & $\begin{array}{l}N \\
N \\
N \\
N \\
N\end{array}$ & $\begin{array}{r}<5 \\
10 \\
5 \\
N \\
20\end{array}$ & $\begin{array}{l}\mathbf{N} \\
N \\
N \\
N \\
N\end{array}$ & $\begin{array}{r}200 \\
500 \\
200 \\
1,000 \\
N\end{array}$ & $\begin{array}{r}15 \\
30 \\
50 \\
15 \\
100\end{array}$ & $\begin{array}{l}\mathbf{N} \\
\mathbf{N} \\
\mathbf{N} \\
\mathbf{N} \\
\mathbf{N}\end{array}$ & $\begin{array}{l}30 \\
20 \\
20 \\
10 \\
30\end{array}$ & $\begin{array}{r}N \\
N \\
N \\
N \\
<200\end{array}$ & $\begin{array}{r}20 \\
50 \\
100 \\
10 \\
50\end{array}$ & $\begin{array}{l}\text { N } \\
N \\
N \\
N \\
N\end{array}$ & $\begin{array}{l}.25 \\
1.00 \\
N\end{array}$ & $\begin{array}{c}20.0 \\
10.0 \\
10.0 \\
N \\
N\end{array}$ \\
\hline
\end{tabular}


TABLE 4--RESULTS OF ANALYSES, DETROIT MINING DISTRICT, UTAH--Continued

\begin{tabular}{|c|c|c|c|c|c|c|c|c|c|c|}
\hline Sample & $\begin{array}{l}\text { BI -PPM } \\
\text { ICP }\end{array}$ & $\begin{array}{l}\text { CD-PPM } \\
I C P\end{array}$ & $\begin{array}{l}\text { SB-PPM } \\
\text { ICP }\end{array}$ & $\begin{array}{l}Z N-P P M \\
I C P\end{array}$ & $\begin{array}{c}\text { Na-pct. } \\
s\end{array}$ & $\begin{array}{c}\text { P-pct. } \\
\text { s }\end{array}$ & $\begin{array}{c}\text { Ga-ppm } \\
\text { s }\end{array}$ & $\begin{array}{c}\text { Ge-ppm } \\
\text { s }\end{array}$ & $\begin{array}{l}\text { HG-PPM } \\
\text { CV }\end{array}$ & $\begin{array}{l}\text { F\% } \\
\text { ISE }\end{array}$ \\
\hline $\begin{array}{l}8 D H 3043 A \\
80 H 3043 B \\
80 H 3043 C \\
70 H 3073 A \\
7 D H 3073 B\end{array}$ & $\begin{array}{r}<2.00 \\
<2.00 \\
<2.00 \\
2.00 \\
2.00\end{array}$ & $\begin{array}{l}<.100 \\
<.100 \\
<.100 \\
.200 \\
.100\end{array}$ & $\begin{array}{c}<2.00 \\
5.00 \\
8.00 \\
N \\
N\end{array}$ & $\begin{array}{r}<2.00 \\
<2.00 \\
<2.00 \\
15.00 \\
5.00\end{array}$ & $\begin{array}{l}N \\
N \\
N \\
\cdots \\
\cdots\end{array}$ & $\begin{array}{r}.2 \\
<.2 \\
<.2 \\
.- \\
-.\end{array}$ & $\begin{array}{l}15 \\
15 \\
10 \\
-- \\
\cdots\end{array}$ & $\begin{array}{c}N \\
N \\
N \\
-- \\
--\end{array}$ & $\begin{array}{l}N \\
.04 \\
.02 \\
-. \\
.-\end{array}$ & $\begin{array}{l}.20 \\
.40 \\
.30 \\
.- \\
.-\end{array}$ \\
\hline $\begin{array}{l}70 H 3074 A \\
7 D A 3074 B \\
7 D H 3074 C \\
70 H 3074 D \\
70 H 3075\end{array}$ & $\begin{array}{l}1.00 \\
2.00 \\
1.00 \\
N \\
1.00\end{array}$ & $\begin{array}{l}N \\
N \\
.200 \\
N \\
.100\end{array}$ & $\begin{array}{c}2.00 \\
N \\
N \\
N \\
N\end{array}$ & $\begin{array}{c}5.00 \\
N^{N} \\
15.00 \\
10.00\end{array}$ & $\begin{array}{l}-- \\
\cdots \\
\cdots \\
\cdots\end{array}$ & $\begin{array}{l}\ldots \\
\cdots \\
\cdots \\
\cdots\end{array}$ & $\begin{array}{l}-. \\
-- \\
\cdots \\
--\end{array}$ & $\begin{array}{l}-- \\
\cdots \\
-- \\
-- \\
--\end{array}$ & $\begin{array}{l}\ldots \\
\cdots \\
\cdots \\
\cdots \\
-.\end{array}$ & $\begin{array}{l}\ldots \\
\cdots \\
-- \\
\cdots\end{array}$ \\
\hline $\begin{array}{l}70 H 3076 \\
70 H 3079 \\
70 H 3080 A \\
70 H 3080 B \\
7 D H 3082 A\end{array}$ & $\begin{array}{c}10.00 \\
N \\
N \\
N \\
3.00\end{array}$ & $\begin{array}{l}.200 \\
.200 \\
.200 \\
.400\end{array}$ & $\begin{array}{c}4.00 \\
N \\
N \\
10.00 \\
300.00\end{array}$ & $\begin{array}{c}5.00 \\
N \\
110.00 \\
550.00 \\
110.00\end{array}$ & $\begin{array}{l}-. \\
\cdots \\
\cdots \\
\cdots\end{array}$ & $\begin{array}{l}\ldots \\
\cdots \\
\cdots \\
\cdots \\
-.\end{array}$ & $\begin{array}{l}\cdots \\
\cdots \\
\cdots \\
--\end{array}$ & $\begin{array}{l}-. \\
\cdots \\
\cdots \\
\cdots\end{array}$ & $\begin{array}{l}-- \\
\cdots \\
-- \\
\cdots\end{array}$ & $\begin{array}{l}\ldots \\
-- \\
\cdots \\
\cdots \\
-.\end{array}$ \\
\hline $\begin{array}{l}70 H 3082 B \\
70 H 3082 C \\
70 H 3083 A \\
70 H 3083 B \\
70 H 3083 C\end{array}$ & $\begin{array}{r}<1.00 \\
92.00 \\
1.00 \\
7.00 \\
1.00\end{array}$ & $\begin{array}{l}.100 \\
.100 \\
.200 \\
.100 \\
.100\end{array}$ & $\begin{array}{l}N \\
N \\
N \\
4.00 \\
N\end{array}$ & $\begin{array}{l}10.00 \\
<5.00 \\
25.00 \\
20.00 \\
<5.00\end{array}$ & $\begin{array}{l}- \\
\cdots \\
\cdots \\
\cdots \\
--\end{array}$ & $\begin{array}{l}\ldots \\
\cdots \\
\cdots \\
\cdots\end{array}$ & $\begin{array}{l}-- \\
\cdots \\
\cdots \\
\cdots\end{array}$ & $\begin{array}{l}\cdots \\
\cdots \\
\cdots \\
\cdots\end{array}$ & $\begin{array}{l}-. \\
\cdots \\
\cdots \\
--\end{array}$ & $\begin{array}{l}-- \\
-- \\
-- \\
-- \\
--\end{array}$ \\
\hline $\begin{array}{l}70 \mathrm{H} 3084 \\
70 \mathrm{H} 3085 \mathrm{~A} \\
7 \mathrm{OH} 3085 \mathrm{~B} \\
7 \mathrm{OH} 3086 \mathrm{~A} \\
7 \mathrm{OH} 3086 \mathrm{~B}\end{array}$ & $\begin{array}{r}6.00 \\
590.00 \\
2.00 \\
1.00 \\
4.00\end{array}$ & $\begin{array}{r}1.200 \\
.200 \\
.100 \\
.100 \\
.200\end{array}$ & $\begin{array}{c}<2.00 \\
900.00 \\
4.00 \\
N \\
N\end{array}$ & $\begin{array}{c}15.00 \\
10.00 \\
55.00 \\
N \\
10.00\end{array}$ & $\begin{array}{l}-. \\
\cdots \\
-- \\
-.\end{array}$ & $\begin{array}{l}-. \\
-- \\
-- \\
--\end{array}$ & $\begin{array}{l}\cdots \\
\cdots \\
\cdots \\
\cdots\end{array}$ & $\begin{array}{l}\ldots \\
\cdots \\
\cdots \\
\cdots\end{array}$ & $\begin{array}{l}-. \\
-- \\
-- \\
--\end{array}$ & $\begin{array}{l}\ldots \\
\ldots \\
\cdots \\
\cdots \\
\cdots\end{array}$ \\
\hline $\begin{array}{l}70 H 3086 C \\
70 H 3087 \\
70 H 3088 A \\
70 H 3088 B \\
70 H 3089 A\end{array}$ & $\begin{array}{l}1.00 \\
N \\
N \\
1.00 \\
1.00\end{array}$ & $\begin{array}{l}1.500 \\
.100 \\
.100 \\
N \\
N\end{array}$ & $\begin{array}{l}N \\
N \\
N \\
N \\
N\end{array}$ & $\begin{array}{c}430.00 \\
N \\
10.00 \\
5.00 \\
N\end{array}$ & $\begin{array}{l}\ldots \\
\cdots \\
\cdots \\
\cdots \\
--\end{array}$ & $\begin{array}{l}-. \\
\cdots \\
\cdots \\
\cdots\end{array}$ & $\begin{array}{l}\ldots \\
\cdots \\
\cdots \\
\cdots\end{array}$ & $\begin{array}{l}\cdots \\
\cdots \\
\cdots \\
\cdots\end{array}$ & $\begin{array}{l}-. \\
\cdots \\
-- \\
--\end{array}$ & $\begin{array}{l}- \\
\cdots \\
\cdots \\
\cdots \\
--\end{array}$ \\
\hline $\begin{array}{l}\text { 7DH3089B } \\
70 H 3127 \\
70 H 3128 \\
70 H 3185 A \\
70 H 3185 B\end{array}$ & $\begin{array}{l}1.00 \\
<1.00 \\
1.00 \\
N \\
N\end{array}$ & $\begin{array}{l}N \\
.100 \\
N \\
N \\
N\end{array}$ & $\begin{array}{l}2.00 \\
N \\
N \\
N\end{array}$ & $\begin{array}{c}N \\
N \\
50.00 \\
<5.00 \\
75.00\end{array}$ & $\begin{array}{l}-. \\
-- \\
-- \\
--\end{array}$ & $\begin{array}{l}\ldots \\
\cdots \\
\ldots \\
\ldots\end{array}$ & $\begin{array}{l}-. \\
- \\
-- \\
--\end{array}$ & $\begin{array}{l}\ldots \\
\cdots \\
\cdots \\
\cdots\end{array}$ & $\begin{array}{l}-. \\
- \\
- \\
-.\end{array}$ & $\begin{array}{l}- \\
-- \\
\cdots \\
-- \\
-\end{array}$ \\
\hline $\begin{array}{l}70 H 3186 \\
70 H 3188 A \\
70 H 3188 B \\
70 H 3188 C \\
70 H 3190\end{array}$ & $\begin{array}{c}N \\
N \\
N \\
N \\
<1.00\end{array}$ & $\begin{array}{l}N \\
N \\
.300 \\
N \\
N\end{array}$ & $\begin{array}{l}N \\
N \\
N \\
N \\
N\end{array}$ & $\begin{array}{c}5.00 \\
10.00 \\
15.00 \\
N \\
30.00\end{array}$ & $\begin{array}{l}\cdots \\
\cdots \\
\cdots \\
\cdots\end{array}$ & $\begin{array}{l}\ldots \\
\cdots \\
\cdots \\
\cdots\end{array}$ & $\begin{array}{l}-- \\
-- \\
-- \\
-\end{array}$ & $\begin{array}{l}\ldots \\
\cdots \\
\cdots \\
\cdots\end{array}$ & $\begin{array}{l}-. \\
-- \\
\cdots \\
--\end{array}$ & $\begin{array}{l}-- \\
-- \\
-- \\
-- \\
\ldots\end{array}$ \\
\hline $\begin{array}{l}\text { 70H3191A } \\
\text { 70H3191B } \\
\text { 70H3192 } \\
\text { TOH3193 } \\
\text { 7OH3194 }\end{array}$ & $\begin{array}{l}N \\
N \\
3.00 \\
1.00 \\
N\end{array}$ & $\begin{array}{l}1.600 \\
N \\
\dot{N}_{N}^{100}\end{array}$ & $\begin{array}{l}4.00 \\
N \\
N \\
N \\
N\end{array}$ & $\begin{array}{r}25.00 \\
10.00 \\
<5.00 \\
10.00 \\
130.00\end{array}$ & $\begin{array}{l}\cdots \\
\cdots \\
\cdots \\
\cdots\end{array}$ & $\begin{array}{l}\ldots \\
\cdots \\
\cdots \\
--\end{array}$ & $\begin{array}{l}-. \\
- \\
-- \\
--\end{array}$ & $\begin{array}{l}\ldots \\
\cdots \\
\cdots \\
\cdots\end{array}$ & $\begin{array}{l}-. \\
\cdots \\
-- \\
-\end{array}$ & $\begin{array}{l}\ldots \\
-- \\
\cdots \\
\cdots \\
--\end{array}$ \\
\hline
\end{tabular}


TABLE 4--RESULTS OF ANALYSES, DETROIT MINING DISTRICT, UTAH--Cont inued

\begin{tabular}{|c|c|c|c|c|c|c|c|c|c|c|c|}
\hline Sample & Latitude & Long & itud & $\begin{array}{c}\text { Fe-pct } \\
\text { s }\end{array}$ & $\begin{array}{c}\text { Mg-pct. } \\
\text { s }\end{array}$ & $\begin{array}{c}\text { Ca-pct. } \\
\text { s }\end{array}$ & $\begin{array}{c}T i-p c t . \\
s\end{array}$ & $\begin{array}{l}\text { Mn-ppm } \\
\text { s }\end{array}$ & $\begin{array}{c}\text { Ag-ppm } \\
s\end{array}$ & $\begin{array}{l}\text { As-ppm } \\
\text { s }\end{array}$ & $\begin{array}{c}\text { Au-ppm } \\
\text { s }\end{array}$ \\
\hline $\begin{array}{l}\text { 7DH3195 } \\
\text { 7DH3196A } \\
\text { 7DH3196B } \\
\text { 7DH3198 } \\
\text { 7DH3203 }\end{array}$ & $\begin{array}{lll}39 & 31 & 35 \\
39 & 31 & 39 \\
39 & 31 & 39 \\
39 & 31 & 39 \\
39 & 33 & 17\end{array}$ & $\begin{array}{l}113 \\
113 \\
113 \\
113 \\
113\end{array}$ & $\begin{array}{ll}0 & 3 \\
0 & 2 \\
0 & 2 \\
0 & 2 \\
0 & 5\end{array}$ & $\begin{array}{l}<.05 \\
3.00 \\
3.00 \\
1.00 \\
.50\end{array}$ & $\begin{array}{l}.02 \\
.50 \\
.70 \\
.02 \\
.05\end{array}$ & $\begin{array}{r}.05 \\
.05 \\
.05 \\
<.05 \\
.20\end{array}$ & $\begin{array}{l}.200 \\
.200 \\
.200 \\
.100 \\
.300\end{array}$ & $\begin{array}{r}<10 \\
20 \\
10 \\
<10 \\
10\end{array}$ & $\begin{array}{l}N \\
N \\
N \\
N \\
N\end{array}$ & $\begin{array}{l}N \\
N \\
N \\
N \\
N\end{array}$ & $\begin{array}{l}N \\
N \\
N \\
N \\
N\end{array}$ \\
\hline $\begin{array}{l}\text { 7DH3207 } \\
\text { 7DH3212 } \\
\text { 7DH3214 } \\
\text { 7DH3215A } \\
\text { 7DH3215B }\end{array}$ & $\begin{array}{lll}39 & 33 & 25 \\
39 & 33 & 13 \\
39 & 33 & 21 \\
39 & 33 & 22 \\
39 & 33 & 22\end{array}$ & $\begin{array}{l}113 \\
113 \\
113 \\
113 \\
113\end{array}$ & $\begin{array}{ll}2 & \\
2 & 1 \\
2 & 1 \\
2 & 1 \\
2 & 1\end{array}$ & $\begin{array}{r}2.00 \\
5.00 \\
.10 \\
7.00 \\
3.00\end{array}$ & $\begin{array}{l}1.00 \\
1.50 \\
2.00 \\
2.00 \\
3.00\end{array}$ & $\begin{array}{r}1.00 \\
1.50 \\
20.00 \\
2.00 \\
1.00\end{array}$ & $\begin{array}{l}.200 \\
.500 \\
.005 \\
.500 \\
.300\end{array}$ & $\begin{array}{l}700 \\
500 \\
200 \\
700 \\
700\end{array}$ & $\begin{array}{l}N \\
N \\
N \\
N \\
N\end{array}$ & $\begin{array}{l}N \\
N \\
N \\
N \\
N\end{array}$ & $\begin{array}{l}N \\
N \\
N \\
N \\
N\end{array}$ \\
\hline $\begin{array}{l}\text { 7DH3218 } \\
\text { TDH3219A } \\
\text { TDH3219B } \\
\text { TDH3219C } \\
70 H 3220\end{array}$ & $\begin{array}{lll}39 & 33 & 22 \\
39 & 33 & 13 \\
39 & 33 & 13 \\
39 & 33 & 13 \\
39 & 33 & 13\end{array}$ & $\begin{array}{l}113 \\
113 \\
113 \\
113 \\
113\end{array}$ & $\begin{array}{ll}2 & 2 \\
2 & 1 \\
2 & 1 \\
2 & 1 \\
2 & 2\end{array}$ & $\begin{array}{l}3.00 \\
3.00 \\
3.00 \\
1.00 \\
3.00\end{array}$ & $\begin{array}{r}3.00 \\
1.50 \\
3.00 \\
.02 \\
1.00\end{array}$ & $\begin{array}{r}10.00 \\
1.00 \\
.20 \\
.50 \\
2.00\end{array}$ & $\begin{array}{l}.500 \\
.200 \\
.200 \\
.200 \\
.200\end{array}$ & $\begin{array}{r}1,000 \\
500 \\
1,000 \\
70 \\
500\end{array}$ & $\begin{array}{c}N \\
N \\
150.00 \\
500.00 \\
2.00\end{array}$ & $\begin{array}{r}N \\
N \\
<200 \\
5,000 \\
N\end{array}$ & $\begin{array}{l}N \\
N \\
N \\
N \\
N\end{array}$ \\
\hline $\begin{array}{l}70 H 3222 \\
70 H 3224 \\
70 H 3226 \\
70 H 3227 \\
7 D H 3228\end{array}$ & $\begin{array}{lll}39 & 33 & 8 \\
39 & 32 & 47 \\
39 & 33 & 20 \\
39 & 33 & 15 \\
39 & 33 & 19\end{array}$ & $\begin{array}{l}113 \\
113 \\
113 \\
113 \\
113\end{array}$ & $\begin{array}{ll}2 & 3 \\
2 & \\
3 & 1 \\
3 & 1 \\
3 & 1\end{array}$ & $\begin{array}{l}.70 \\
2.00 \\
5.00 \\
5.00 \\
<.05\end{array}$ & $\begin{array}{r}.70 \\
1.00 \\
2.00 \\
2.00 \\
.10\end{array}$ & $\begin{array}{r}.20 \\
.50 \\
2.00 \\
5.00 \\
.50\end{array}$ & $\begin{array}{l}.150 \\
.200 \\
.300 \\
.500 \\
.100\end{array}$ & $\begin{array}{r}100 \\
500 \\
500 \\
1,000 \\
10\end{array}$ & $\begin{array}{l}1.00 \\
N \\
.50 \\
<.50 \\
N\end{array}$ & $\begin{array}{l}N \\
N \\
N \\
N \\
N\end{array}$ & $\begin{array}{l}N \\
N \\
N \\
N \\
N\end{array}$ \\
\hline $\begin{array}{l}70 H 3229 \\
70 H 3231 \\
70 H 3249 \\
80 H 3276 A \\
80 H 3276 B\end{array}$ & $\begin{array}{lll}39 & 33 & 20 \\
39 & 33 & 14 \\
39 & 33 & 20 \\
39 & 34 & 33 \\
39 & 34 & 33\end{array}$ & $\begin{array}{l}113 \\
113 \\
113 \\
113 \\
113\end{array}$ & $\begin{array}{ll}2 & 5 \\
3 & 2 \\
2 & 5 \\
3 & 2 \\
3 & 2\end{array}$ & $\begin{array}{r}10.00 \\
5.00 \\
3.00 \\
2.00 \\
1.50\end{array}$ & $\begin{array}{l}.15 \\
.10 \\
.10 \\
.20 \\
.10\end{array}$ & $\begin{array}{r}1.00 \\
.10 \\
.10 \\
.10 \\
.05\end{array}$ & $\begin{array}{l}.100 \\
.050 \\
.050 \\
.010 \\
.005\end{array}$ & $\begin{array}{r}100 \\
50 \\
>5,000 \\
3,000 \\
50\end{array}$ & $\begin{array}{l}N \\
N \\
N \\
N \\
N\end{array}$ & $\begin{array}{l}N \\
N \\
N \\
N \\
N\end{array}$ & $\begin{array}{l}N \\
N \\
N \\
N \\
N\end{array}$ \\
\hline $\begin{array}{l}80 H 3283 \\
80 H 3284 A \\
80 H 3284 B \\
80 H 3287 A \\
80 H 3287 B\end{array}$ & $\begin{array}{lll}39 & 32 & 39 \\
39 & 32 & 54 \\
39 & 32 & 54 \\
39 & 33 & 36 \\
39 & 33 & 36\end{array}$ & $\begin{array}{l}113 \\
113 \\
113 \\
113 \\
113\end{array}$ & $\begin{array}{l}0 \\
0 \\
0 \\
2 \\
2\end{array}$ & $\begin{array}{r}1.00 \\
7.00 \\
10.00 \\
3.00 \\
.15\end{array}$ & $\begin{array}{r}.30 \\
.05 \\
.15 \\
.20 \\
10.00\end{array}$ & $\begin{array}{r}.07 \\
.07 \\
.20 \\
.20 \\
20.00\end{array}$ & $\begin{array}{r}1.000 \\
.500 \\
.050 \\
.020 \\
<.002\end{array}$ & $\begin{array}{r}20 \\
30 \\
50 \\
200 \\
300\end{array}$ & $\begin{array}{c}N \\
N \\
N \\
N \\
N\end{array}$ & $\begin{array}{r}N \\
N \\
N \\
N, 000 \\
N\end{array}$ & $\begin{array}{l}N \\
N \\
N \\
N \\
N\end{array}$ \\
\hline $\begin{array}{l}80 H 3288 B \\
80 H 3289 B \\
80 H 3291 \\
80 H 3292 B \\
80 H 3294 B\end{array}$ & $\begin{array}{lll}39 & 33 & 33 \\
39 & 33 & 38 \\
39 & 33 & 36 \\
39 & 33 & 28 \\
39 & 33 & 34\end{array}$ & $\begin{array}{l}113 \\
113 \\
113 \\
113 \\
113\end{array}$ & $\begin{array}{l}2 \\
2 \\
2 \\
1 \\
1\end{array}$ & $\begin{array}{c}20.00 \\
7.00 \\
N \\
7.00 \\
5.00\end{array}$ & $\begin{array}{r}.50 \\
.20 \\
.10 \\
5.00 \\
.50\end{array}$ & $\begin{array}{r}.10 \\
.07 \\
>20.00 \\
1.00 \\
.90\end{array}$ & $\begin{array}{r}.150 \\
.100 \\
<.002 \\
.700 \\
.500\end{array}$ & $\begin{array}{r}10 \\
1,000 \\
700 \\
1,000 \\
50\end{array}$ & $\begin{array}{l}2.00 \\
5.00 \\
N \\
N \\
N\end{array}$ & $\begin{array}{r}3,000 \\
700 \\
N \\
N \\
N\end{array}$ & $\begin{array}{l}20 \\
N \\
N \\
N \\
N\end{array}$ \\
\hline $\begin{array}{l}80 H 3294 \mathrm{C} \\
80 \mathrm{H} 3295 \\
80 \mathrm{H} 3296 \\
80 \mathrm{H} 3298 \\
80 \mathrm{H} 3299\end{array}$ & $\begin{array}{lll}39 & 33 & 34 \\
39 & 33 & 29 \\
39 & 33 & 32 \\
39 & 33 & 40 \\
39 & 31 & 51\end{array}$ & $\begin{array}{l}113 \\
113 \\
113 \\
113 \\
113\end{array}$ & $\begin{array}{l}13 \\
13 \\
1 \\
2 \\
0\end{array}$ & $\begin{array}{r}5.00 \\
7.00 \\
.05 \\
3.00 \\
3.00\end{array}$ & $\begin{array}{r}.20 \\
1.50 \\
5.00 \\
7.00 \\
.10\end{array}$ & $\begin{array}{r}<.05 \\
.15 \\
>20.00 \\
20.00 \\
.20\end{array}$ & $\begin{array}{l}.500 \\
.500 \\
.003 \\
.200 \\
1.000\end{array}$ & $\begin{array}{r}30 \\
300 \\
50 \\
200 \\
10\end{array}$ & $\begin{array}{l}\mathbf{N} \\
\mathbf{N} \\
\mathbf{N} \\
\mathbf{N} \\
\mathbf{N}\end{array}$ & $\begin{array}{l}N \\
N \\
N \\
N \\
N\end{array}$ & $\begin{array}{l}N \\
N \\
N \\
N \\
N\end{array}$ \\
\hline $\begin{array}{l}80 H 3300 \\
80 H 3302 A \\
80 H 3302 B \\
80 H 3303 \\
80 H 3305\end{array}$ & $\begin{array}{rrr}39 & 31 & 46 \\
39 & 32 & 1 \\
39 & 32 & 1 \\
39 & 32 & 4 \\
39 & 32 & 19\end{array}$ & $\begin{array}{l}113 \\
112 \\
112 \\
112 \\
112\end{array}$ & $\begin{array}{c}0 \\
59 \\
59 \\
59 \\
59\end{array}$ & $\begin{array}{r}2.00 \\
2.00 \\
2.00 \\
1.00 \\
20.00\end{array}$ & $\begin{array}{l}.70 \\
.50 \\
.70 \\
.20 \\
.20\end{array}$ & $\begin{array}{r}.05 \\
.15 \\
.10 \\
2.00 \\
.05\end{array}$ & $\begin{array}{r}.500 \\
.200 \\
1.000 \\
.200 \\
.070\end{array}$ & $\begin{array}{r}70 \\
20 \\
20 \\
500 \\
50\end{array}$ & $\begin{array}{l}\mathbf{N} \\
\mathbf{N} \\
\mathbf{N} \\
\mathbf{N} \\
\mathbf{N}\end{array}$ & $\begin{array}{r}N \\
N \\
1,500 \\
N \\
500\end{array}$ & $\begin{array}{l}N \\
N \\
N \\
N \\
N\end{array}$ \\
\hline
\end{tabular}


TABLE 4--RESULTS OF ANALYSES, DETROIT MINING DISTRICT, UTAH--Continued

\begin{tabular}{|c|c|c|c|c|c|c|c|c|c|c|c|c|c|}
\hline Sample & $\begin{array}{c}\text { B-ppm } \\
\text { s }\end{array}$ & $\begin{array}{c}\text { Ba-ppm } \\
s\end{array}$ & $\begin{array}{c}\text { Be-ppm } \\
s\end{array}$ & $\begin{array}{c}\text { Bi-ppm } \\
\mathrm{s}\end{array}$ & $\begin{array}{c}\text { Cd-ppm } \\
s\end{array}$ & $\begin{array}{c}\text { Co-ppm } \\
\mathrm{s}\end{array}$ & $\begin{array}{c}\mathrm{Cr}-\mathrm{ppm} \\
\mathrm{s}\end{array}$ & $\begin{array}{c}\text { Cu-ppm } \\
s\end{array}$ & $\begin{array}{l}\text { La-ppn } \\
\quad s\end{array}$ & $\begin{array}{c}\text { Mo-ppm } \\
\mathrm{s}\end{array}$ & $\begin{array}{c}\text { Nb-ppm } \\
s\end{array}$ & $\begin{array}{c}\mathrm{Ni}-\mathrm{ppm} \\
\mathrm{s}\end{array}$ & $\begin{array}{l}\text { Pb-ppm } \\
s\end{array}$ \\
\hline $\begin{array}{l}\text { 7DH3195 } \\
70 H 3196 A \\
7 D H 31968 \\
7 D H 3198 \\
70 H 3203\end{array}$ & $\begin{array}{l}15 \\
50 \\
70 \\
15 \\
10\end{array}$ & $\begin{array}{r}20 \\
500 \\
1,000 \\
200 \\
300\end{array}$ & $\begin{array}{c}N \\
1.5 \\
1.5 \\
N \\
<1.0\end{array}$ & $\begin{array}{l}N \\
N \\
N \\
N \\
N\end{array}$ & $\begin{array}{l}N \\
N \\
N \\
N \\
N\end{array}$ & $\begin{array}{r}<10 \\
50 \\
30 \\
<10 \\
<10\end{array}$ & $\begin{array}{l}10 \\
30 \\
30 \\
10 \\
50\end{array}$ & $\begin{array}{l}<5 \\
20 \\
20 \\
20 \\
20\end{array}$ & $\begin{array}{r}<20 \\
<20 \\
70 \\
N \\
50\end{array}$ & $\begin{array}{r}N \\
N \\
10 \\
5 \\
5\end{array}$ & $\begin{array}{r}<20 \\
<20 \\
<20 \\
N \\
<20\end{array}$ & $\begin{array}{l}<5 \\
50 \\
50 \\
15 \\
<5\end{array}$ & $\begin{array}{r}<10 \\
50 \\
20 \\
300 \\
200\end{array}$ \\
\hline $\begin{array}{l}\text { TDH3207 } \\
\text { 7DH3212 } \\
7 \mathrm{DH} 3214 \\
\text { TDH3215A } \\
7 \mathrm{DH} 3215 \mathrm{~B}\end{array}$ & $\begin{array}{r}<10 \\
<10 \\
N \\
10 \\
<10\end{array}$ & $\begin{array}{r}500 \\
700 \\
N \\
700 \\
500\end{array}$ & $\begin{array}{r}<1.0 \\
<1.0 \\
N \\
<1.0 \\
<1.0\end{array}$ & $\begin{array}{l}\mathbf{N} \\
\mathbf{N} \\
\mathbf{N} \\
\mathbf{N} \\
\mathbf{N}\end{array}$ & $\begin{array}{l}N \\
N \\
N \\
N \\
N\end{array}$ & $\begin{array}{r}10 \\
30 \\
N \\
50 \\
30\end{array}$ & $\begin{array}{r}N \\
<10 \\
<10 \\
<10 \\
<10\end{array}$ & $\begin{array}{l}<5 \\
15 \\
<5 \\
15 \\
20\end{array}$ & $\begin{array}{r}50 \\
50 \\
N \\
<20 \\
70\end{array}$ & $\begin{array}{l}N \\
N \\
N \\
N \\
N\end{array}$ & $\begin{array}{r}N \\
N \\
N \\
<20 \\
N\end{array}$ & $\begin{array}{r}<5 \\
5 \\
N \\
7 \\
10\end{array}$ & $\begin{array}{r}50 \\
50 \\
<10 \\
50 \\
70\end{array}$ \\
\hline $\begin{array}{l}7 D H 3218 \\
70 H 3219 A \\
70 H 32198 \\
7 D H 3219 C \\
7 D H 3220\end{array}$ & $\begin{array}{l}10 \\
15 \\
15 \\
10 \\
10\end{array}$ & $\begin{array}{r}1,000 \\
500 \\
200 \\
70 \\
50\end{array}$ & $\begin{array}{r}<1.0 \\
<1.0 \\
1.5 \\
2.0 \\
<1.0\end{array}$ & $\begin{array}{r}N \\
N \\
N \\
>1,000 \\
150\end{array}$ & $\begin{array}{r}N \\
N \\
N \\
500 \\
N\end{array}$ & $\begin{array}{l}20 \\
20 \\
<5 \\
<5 \\
20\end{array}$ & $\begin{array}{l}<10 \\
<10 \\
<10 \\
<10 \\
<10\end{array}$ & $\begin{array}{r}7 \\
10 \\
1,000 \\
>20,000 \\
100\end{array}$ & $\begin{array}{r}100 \\
50 \\
70 \\
<20 \\
<20\end{array}$ & $\begin{array}{l}N \\
N \\
N \\
N \\
<5\end{array}$ & $\begin{array}{r}N \\
N \\
N \\
<20 \\
N\end{array}$ & $\begin{array}{l}<5 \\
10 \\
10 \\
15 \\
10\end{array}$ & $\begin{array}{r}100 \\
70 \\
100 \\
1,000 \\
70\end{array}$ \\
\hline $\begin{array}{l}\text { 7DH3222 } \\
7 D H 3224 \\
\text { 7DH3226 } \\
70 H 3227 \\
7 D H 3228\end{array}$ & $\begin{array}{r}30 \\
<10 \\
<10 \\
<10 \\
10\end{array}$ & $\begin{array}{r}1,000 \\
500 \\
700 \\
1,000 \\
100\end{array}$ & $\begin{array}{c}1.0 \\
N \\
<1.0 \\
1.0 \\
N\end{array}$ & $\begin{array}{r}100 \\
N \\
50 \\
50 \\
<10\end{array}$ & $\begin{array}{l}N \\
N \\
N \\
N \\
N\end{array}$ & $\begin{array}{l}N \\
<5 \\
30 \\
50 \\
<5\end{array}$ & $\begin{array}{l}<10 \\
<10 \\
<10 \\
<10 \\
<10\end{array}$ & $\begin{array}{l}50 \\
<5 \\
50 \\
50 \\
15\end{array}$ & $\begin{array}{r}50 \\
50 \\
50 \\
70 \\
<20\end{array}$ & $\begin{array}{l}N \\
N \\
N \\
N \\
N\end{array}$ & $\begin{array}{r}N \\
N \\
N \\
N \\
<20\end{array}$ & $\begin{array}{r}<5 \\
5 \\
20 \\
50 \\
5\end{array}$ & $\begin{array}{r}70 \\
50 \\
70 \\
70 \\
<10\end{array}$ \\
\hline $\begin{array}{l}\text { 7DH3229 } \\
7 D H 3231 \\
7 D H 3249 \\
8 D H 3276 A \\
8 D H 3276 B\end{array}$ & $\begin{array}{r}<10 \\
20 \\
<10 \\
20 \\
50\end{array}$ & $\begin{array}{r}200 \\
100 \\
N \\
1,000 \\
200\end{array}$ & $\begin{array}{l}N \\
2.0 \\
N \\
1.0 \\
1.0\end{array}$ & $\begin{array}{r}10 \\
N \\
N \\
N \\
N\end{array}$ & $\begin{array}{l}N \\
N \\
N \\
N \\
N\end{array}$ & $\begin{array}{l}<5 \\
<5 \\
70 \\
N \\
N\end{array}$ & $\begin{array}{r}<10 \\
<10 \\
N \\
N \\
N\end{array}$ & $\begin{array}{r}15 \\
10 \\
20 \\
5 \\
5\end{array}$ & $\begin{array}{r}N \\
N \\
N \\
<50 \\
N\end{array}$ & $\begin{array}{r}20 \\
7 \\
100 \\
N \\
N\end{array}$ & $\begin{array}{l}N \\
N \\
N \\
N \\
N\end{array}$ & $\begin{array}{r}<5 \\
15 \\
100 \\
<5 \\
<5\end{array}$ & $\begin{array}{r}150 \\
20 \\
100 \\
<10 \\
<10\end{array}$ \\
\hline $\begin{array}{l}8 D H 3283 \\
8 D H 3284 A \\
8 D H 3284 B \\
8 D H 3287 A \\
8 D H 3287 B\end{array}$ & $\begin{array}{r}20 \\
15 \\
10 \\
15 \\
N\end{array}$ & $\begin{array}{r}300 \\
500 \\
200 \\
50 \\
<20\end{array}$ & $\begin{array}{c}<1.0 \\
N \\
1.5 \\
N \\
N\end{array}$ & $\begin{array}{c}N \\
N \\
N \\
70 \\
N\end{array}$ & $\begin{array}{l}N \\
N \\
N \\
N \\
N\end{array}$ & $\begin{array}{l}N \\
N \\
N \\
N \\
N\end{array}$ & $\begin{array}{r}<10 \\
<10 \\
<10 \\
<10 \\
N\end{array}$ & $\begin{array}{r}5 \\
15 \\
20 \\
1,000 \\
20\end{array}$ & $\begin{array}{r}50 \\
<50 \\
N \\
N \\
N\end{array}$ & $\begin{array}{r}N \\
<5 \\
5 \\
<5 \\
N\end{array}$ & $\begin{array}{l}N \\
N \\
N \\
N \\
N\end{array}$ & $\begin{array}{r}N \\
<5 \\
20 \\
10 \\
N\end{array}$ & $\begin{array}{r}10 \\
20 \\
50 \\
1,000 \\
100\end{array}$ \\
\hline $\begin{array}{l}80 H 3288 B \\
80 H 3289 B \\
8 D H 3291 \\
80 H 3292 B \\
8 D H 3294 \text { B }\end{array}$ & $\begin{array}{r}20 \\
50 \\
N \\
<10 \\
50\end{array}$ & $\begin{array}{r}500 \\
300 \\
20 \\
500 \\
300\end{array}$ & $\begin{array}{l}N \\
N \\
N \\
N \\
N\end{array}$ & $\begin{array}{r}100 \\
50 \\
N \\
N \\
N\end{array}$ & $\begin{array}{l}N \\
N \\
N \\
N \\
N\end{array}$ & $\begin{array}{r}<10 \\
100 \\
N \\
30 \\
N\end{array}$ & $\begin{array}{r}50 \\
<10 \\
N \\
10 \\
70\end{array}$ & $\begin{array}{r}1,500 \\
10,000 \\
<5 \\
50 \\
50\end{array}$ & $\begin{array}{r}N \\
<50 \\
N \\
50 \\
50\end{array}$ & $\begin{array}{r}20 \\
10 \\
N \\
N \\
N\end{array}$ & $\begin{array}{r}N \\
N \\
N \\
N \\
<20\end{array}$ & $\begin{array}{r}20 \\
70 \\
N \\
10 \\
5\end{array}$ & $\begin{array}{r}700 \\
1,000 \\
<10 \\
30 \\
200\end{array}$ \\
\hline $\begin{array}{l}80 H 3294 \mathrm{C} \\
8 D H 3295 \\
80 H 3296 \\
80 H 3298 \\
80 H 3299\end{array}$ & $\begin{array}{r}20 \\
100 \\
N \\
100 \\
10\end{array}$ & $\begin{array}{r}100 \\
500 \\
20 \\
200 \\
700\end{array}$ & $\begin{array}{c}2.0 \\
N \\
N 1.0\end{array}$ & $\begin{array}{l}N \\
N \\
N \\
N \\
N\end{array}$ & $\begin{array}{l}N \\
N \\
N \\
N \\
N\end{array}$ & $\begin{array}{r}20 \\
15 \\
N \\
10 \\
20\end{array}$ & $\begin{array}{r}100 \\
100 \\
<10 \\
50 \\
100\end{array}$ & $\begin{array}{l}50 \\
50 \\
<5 \\
30 \\
10\end{array}$ & $\begin{array}{r}70 \\
50 \\
N \\
<50 \\
70\end{array}$ & $\begin{array}{l}10 \\
N \\
N \\
N \\
N\end{array}$ & $\begin{array}{r}<20 \\
N \\
N \\
N \\
<20\end{array}$ & $\begin{array}{r}30 \\
50 \\
N \\
50 \\
50\end{array}$ & $\begin{array}{r}20 \\
10 \\
15 \\
<10 \\
300\end{array}$ \\
\hline $\begin{array}{l}80 H 3300 \\
8 D H 3302 A \\
80 H 3302 B \\
80 H 3303 \\
8 D H 3305\end{array}$ & $\begin{array}{r}50 \\
10 \\
30 \\
15 \\
<10\end{array}$ & $\begin{array}{r}1,000 \\
300 \\
300 \\
500 \\
300\end{array}$ & $\begin{array}{r}1.0 \\
<1.0 \\
1.0 \\
N \\
N\end{array}$ & $\begin{array}{l}N \\
N \\
N \\
N \\
N\end{array}$ & $\begin{array}{l}N \\
N \\
N \\
N \\
N\end{array}$ & $\begin{array}{r}N \\
N \\
15 \\
N \\
N\end{array}$ & $\begin{array}{r}100 \\
10 \\
150 \\
15 \\
70\end{array}$ & $\begin{array}{l}50 \\
15 \\
20 \\
10 \\
30\end{array}$ & $\begin{array}{r}70 \\
<50 \\
50 \\
N \\
N\end{array}$ & $\begin{array}{l}N \\
N \\
N \\
5 \\
N\end{array}$ & $\begin{array}{r}<20 \\
N \\
N \\
N \\
N\end{array}$ & $\begin{array}{r}10 \\
5 \\
20 \\
5 \\
20\end{array}$ & $\begin{array}{r}70 \\
10 \\
<10 \\
500 \\
10\end{array}$ \\
\hline
\end{tabular}


TABLE 4--RESULTS OF ANALYSES, DETROIT MINING DISTRICT, UTAH--Cont inued

\begin{tabular}{|c|c|c|c|c|c|c|c|c|c|c|c|c|}
\hline Sample & $\begin{array}{c}\text { Sb-ppm } \\
\text { s }\end{array}$ & $\begin{array}{c}\text { Sc-ppm } \\
\mathbf{s}\end{array}$ & $\begin{array}{c}\text { Sn-ppm } \\
s\end{array}$ & $\begin{array}{c}\text { Sr-ppm } \\
\text { s }\end{array}$ & $\begin{array}{c}\text { V-ppm } \\
\text { s }\end{array}$ & $\begin{array}{c}\text { W-ppm } \\
\mathbf{s}\end{array}$ & $\begin{array}{c}Y \text {-ppm } \\
\text { s }\end{array}$ & $\begin{array}{c}\text { Zn-ppm } \\
s\end{array}$ & $\begin{array}{c}\mathrm{Zr}-\mathrm{ppm} \\
\mathrm{s}\end{array}$ & $\begin{array}{c}\text { Th-ppm } \\
\mathbf{s}\end{array}$ & $\begin{array}{c}\text { Au-ppm } \\
\text { aa }\end{array}$ & $\begin{array}{l}\text { AS-PPM } \\
\text { ICP }\end{array}$ \\
\hline $\begin{array}{l}70 H 3195 \\
70 H 3196 A \\
70 H 31968 \\
70 H 3198 \\
70 H 3203\end{array}$ & $\begin{array}{l}N \\
N \\
N \\
N \\
N\end{array}$ & $\begin{array}{r}N \\
15 \\
20 \\
<5 \\
10\end{array}$ & $\begin{array}{l}N \\
N \\
N \\
N \\
N\end{array}$ & $\begin{array}{r}200 \\
N \\
N \\
300 \\
300\end{array}$ & $\begin{array}{r}50 \\
70 \\
70 \\
50 \\
100\end{array}$ & $\begin{array}{l}N \\
N \\
N \\
N \\
N\end{array}$ & $\begin{array}{r}N \\
30 \\
30 \\
N \\
<10\end{array}$ & $\begin{array}{r}<200 \\
<200 \\
N \\
N \\
<200\end{array}$ & $\begin{array}{r}70 \\
150 \\
100 \\
100 \\
200\end{array}$ & $\begin{array}{l}N \\
N \\
N \\
N \\
N\end{array}$ & $\begin{array}{l}.35 \\
N \\
.05 \\
.30 \\
.05\end{array}$ & $\begin{array}{c}N \\
10.0 \\
20.0 \\
20.0 \\
N\end{array}$ \\
\hline $\begin{array}{l}7 D H 3207 \\
7 D H 3212 \\
7 D H 3214 \\
7 D H 3215 A \\
70 H 3215 B\end{array}$ & $\begin{array}{l}N \\
N \\
N \\
N \\
N\end{array}$ & $\begin{array}{r}15 \\
20 \\
N \\
30 \\
20\end{array}$ & $\begin{array}{l}N \\
N \\
N \\
N \\
N\end{array}$ & $\begin{array}{l}500 \\
500 \\
200 \\
500 \\
500\end{array}$ & $\begin{array}{r}70 \\
200 \\
10 \\
200 \\
100\end{array}$ & $\begin{array}{l}N \\
N \\
N \\
N \\
N\end{array}$ & $\begin{array}{r}20 \\
30 \\
N \\
30 \\
20\end{array}$ & $\begin{array}{r}<200 \\
<200 \\
N \\
<200 \\
<200\end{array}$ & $\begin{array}{l}100 \\
200 \\
<10 \\
200 \\
150\end{array}$ & $\begin{array}{l}N \\
N \\
N \\
N \\
N\end{array}$ & $\begin{array}{l}N \\
N \\
N \\
N \\
N\end{array}$ & $\begin{array}{c}10.0 \\
N \\
N \\
N \\
N\end{array}$ \\
\hline $\begin{array}{l}7 D H 3218 \\
7 D H 3219 A \\
7 D H 3219 B \\
7 D H 3219 C \\
7 D H 3220\end{array}$ & $\begin{array}{r}N \\
N \\
100 \\
>10,000 \\
N\end{array}$ & $\begin{array}{r}20 \\
10 \\
7 \\
7 \\
15\end{array}$ & $\begin{array}{r}N \\
N \\
N \\
1,000 \\
100\end{array}$ & $\begin{array}{r}1,000 \\
200 \\
N \\
300 \\
300\end{array}$ & $\begin{array}{l}150 \\
100 \\
100 \\
200 \\
150\end{array}$ & $\begin{array}{l}N \\
N \\
N \\
N \\
N\end{array}$ & $\begin{array}{l}50 \\
20 \\
20 \\
50 \\
20\end{array}$ & $\begin{array}{r}N \\
<200 \\
500 \\
1,000 \\
N\end{array}$ & $\begin{array}{l}200 \\
100 \\
100 \\
100 \\
150\end{array}$ & $\begin{array}{l}N \\
N \\
N \\
N \\
N\end{array}$ & $\begin{array}{l}N \\
N \\
<.05 \\
3.80 \\
N\end{array}$ & $\begin{array}{c}N \\
N \\
300.0 \\
800.0 \\
N\end{array}$ \\
\hline $\begin{array}{l}\text { TOH3222 } \\
\text { TDH3224 } \\
\text { 7DH3226 } \\
\text { TDH3227 } \\
\text { TOH3228 }\end{array}$ & $\begin{array}{l}N \\
N \\
N \\
N \\
N\end{array}$ & $\begin{array}{r}5 \\
10 \\
20 \\
20 \\
N\end{array}$ & $\begin{array}{r}30 \\
N \\
20 \\
10 \\
N\end{array}$ & $\begin{array}{l}200 \\
100 \\
500 \\
700 \\
300\end{array}$ & $\begin{array}{r}50 \\
70 \\
150 \\
150 \\
50\end{array}$ & $\begin{array}{l}N \\
N \\
N \\
N \\
N\end{array}$ & $\begin{array}{r}10 \\
15 \\
30 \\
30 \\
N\end{array}$ & $\begin{array}{r}N \\
N \\
<200 \\
<200 \\
N\end{array}$ & $\begin{array}{l}150 \\
150 \\
150 \\
150 \\
100\end{array}$ & $\begin{array}{l}N \\
N \\
N \\
N \\
N\end{array}$ & $\begin{array}{l}N \\
N \\
N \\
N \\
N\end{array}$ & $\begin{array}{l}N \\
N \\
N \\
N \\
N\end{array}$ \\
\hline $\begin{array}{l}70 H 3229 \\
70 H 3231 \\
70 H 3249 \\
80 H 3276 A \\
80 H 3276 B\end{array}$ & $\begin{array}{l}N \\
N \\
N \\
N \\
N\end{array}$ & $\begin{array}{r}<5 \\
5 \\
5 \\
N \\
N\end{array}$ & $\begin{array}{l}N \\
N \\
N \\
N \\
N\end{array}$ & $\begin{array}{r}100 \\
200 \\
300 \\
N \\
N\end{array}$ & $\begin{array}{l}50 \\
30 \\
70 \\
20 \\
20\end{array}$ & $\begin{array}{l}N \\
N \\
N \\
N \\
N\end{array}$ & $\begin{array}{r}10 \\
15 \\
30 \\
N \\
N\end{array}$ & $\begin{array}{r}N \\
<200 \\
<200 \\
N \\
N\end{array}$ & $\begin{array}{r}100 \\
50 \\
100 \\
<10 \\
<10\end{array}$ & $\begin{array}{l}N \\
N \\
N \\
N \\
N\end{array}$ & $\begin{array}{l}N \\
N \\
N \\
<.05 \\
<.05\end{array}$ & $\begin{array}{r}40.0 \\
20.0 \\
900.0 \\
<5.0 \\
8.0\end{array}$ \\
\hline $\begin{array}{l}80 H 3283 \\
80 \text { H3284A } \\
80 \text { H3284B } \\
80 \text { H3287A } \\
80 \text { H3287B }\end{array}$ & $\begin{array}{r}N \\
N \\
N \\
<100 \\
N\end{array}$ & $\begin{array}{l}10 \\
<5 \\
N \\
N \\
N\end{array}$ & $\begin{array}{r}N \\
N \\
N \\
20 \\
N\end{array}$ & $\begin{array}{r}200 \\
100 \\
N \\
N \\
N\end{array}$ & $\begin{array}{r}150 \\
100 \\
20 \\
50 \\
<10\end{array}$ & $\begin{array}{l}N \\
N \\
N \\
N \\
N\end{array}$ & $\begin{array}{r}20 \\
<10 \\
N \\
100 \\
N\end{array}$ & $\begin{array}{r}N \\
N \\
N \\
10,000 \\
N\end{array}$ & $\begin{array}{r}200 \\
150 \\
100 \\
<10 \\
N\end{array}$ & $\begin{array}{l}N \\
N \\
N \\
N \\
N\end{array}$ & $\begin{array}{l}<.05 \\
<.05 \\
<.05 \\
.70 \\
.05\end{array}$ & $\begin{array}{r}<5.0 \\
<5.0 \\
17.0 \\
780.0 \\
<5.0\end{array}$ \\
\hline $\begin{array}{l}\text { 80H3288B } \\
80 H 3289 B \\
80 H 3291 \\
80 H 3292 B \\
80 H 3294 B\end{array}$ & $\begin{array}{r}500 \\
100 \\
N \\
N \\
N\end{array}$ & $\begin{array}{r}N \\
<5 \\
N \\
20 \\
15\end{array}$ & $\begin{array}{r}>1,000 \\
30 \\
N \\
N \\
N\end{array}$ & $\begin{array}{r}500 \\
N \\
500 \\
300 \\
N\end{array}$ & $\begin{array}{r}200 \\
20 \\
<10 \\
150 \\
100\end{array}$ & $\begin{array}{l}N \\
N \\
N \\
N \\
N\end{array}$ & $\begin{array}{r}50 \\
30 \\
N \\
20 \\
50\end{array}$ & $\begin{array}{r}500 \\
1,000 \\
N \\
N \\
N\end{array}$ & $\begin{array}{r}150 \\
50 \\
N \\
150 \\
100\end{array}$ & $\begin{array}{l}N \\
N \\
N \\
N \\
N\end{array}$ & $\begin{array}{r}20.00 \\
1.00 \\
.05 \\
<.05 \\
.10\end{array}$ & $\begin{array}{r}2,300.0 \\
790.0 \\
<5.0 \\
11.0 \\
28.0\end{array}$ \\
\hline $\begin{array}{l}80 H 3294 \mathrm{C} \\
80 \mathrm{H} 3295 \\
80 \mathrm{H} 3296 \\
80 \mathrm{H} 3298 \\
80 \mathrm{H} 3299\end{array}$ & $\begin{array}{l}N \\
N \\
N \\
N \\
N\end{array}$ & $\begin{array}{r}10 \\
15 \\
N \\
10 \\
10\end{array}$ & $\begin{array}{l}N \\
N \\
N \\
N \\
N\end{array}$ & $\begin{array}{r}500 \\
N \\
300 \\
300 \\
500\end{array}$ & $\begin{array}{r}100 \\
100 \\
<10 \\
50 \\
200\end{array}$ & $\begin{array}{l}N \\
N \\
N \\
N \\
N\end{array}$ & $\begin{array}{r}<10 \\
30 \\
N \\
15 \\
20\end{array}$ & $\begin{array}{l}N \\
N \\
N \\
N \\
N\end{array}$ & $\begin{array}{r}100 \\
100 \\
N \\
100 \\
200\end{array}$ & $\begin{array}{l}N \\
N \\
N \\
N \\
N\end{array}$ & $\begin{array}{l}<.05 \\
<.05 \\
<.05 \\
<.05 \\
<.05\end{array}$ & $\begin{array}{l}70.0 \\
<5.0 \\
<5.0 \\
<5.0 \\
19.0\end{array}$ \\
\hline $\begin{array}{l}80 H 3300 \\
80 H 3302 A \\
80 H 3302 B \\
80 H 3303 \\
80 H 3305\end{array}$ & $\begin{array}{r}N \\
N \\
N \\
<100 \\
N\end{array}$ & $\begin{array}{r}15 \\
5 \\
10 \\
N \\
10\end{array}$ & $\begin{array}{l}N \\
N \\
N \\
N \\
N\end{array}$ & $\begin{array}{r}100 \\
500 \\
<100 \\
1,000 \\
300\end{array}$ & $\begin{array}{r}100 \\
70 \\
150 \\
50 \\
100\end{array}$ & $\begin{array}{l}N \\
N \\
N \\
N \\
N\end{array}$ & $\begin{array}{r}20 \\
10 \\
20 \\
N \\
200\end{array}$ & $\begin{array}{l}N \\
N \\
N \\
N \\
N\end{array}$ & $\begin{array}{l}200 \\
200 \\
200 \\
150 \\
100\end{array}$ & $\begin{array}{l}\mathbf{N} \\
\mathbf{N} \\
\mathbf{N} \\
\mathbf{N} \\
\mathbf{N}\end{array}$ & $\begin{array}{l}<.05 \\
<.05 \\
<.05 \\
.60 \\
<.05\end{array}$ & $\begin{array}{r}84.0 \\
31.0 \\
1,400.0 \\
86.0 \\
570.0\end{array}$ \\
\hline
\end{tabular}


TABLE 4--RESULTS OF ANALYSES, DETROIT MINING DISTRICT, UTAH--Continued

\begin{tabular}{|c|c|c|c|c|c|c|c|c|c|c|}
\hline Sample & $\begin{array}{l}B I-P P M \\
I C P\end{array}$ & $\begin{array}{l}C D-P P M \\
I C P\end{array}$ & $\begin{array}{l}\text { SB-PPM } \\
\text { ICP }\end{array}$ & $\begin{array}{l}Z N-P P M \\
I C P\end{array}$ & $\begin{array}{c}\mathrm{Na}-\mathrm{pct} \\
\mathbf{s}\end{array}$ & $\begin{array}{c}\text { p-pct. } \\
s\end{array}$ & $\begin{array}{c}\text { Ga-ppm } \\
\mathrm{s}\end{array}$ & $\begin{array}{l}\text { Ge-ppm } \\
\text { s }\end{array}$ & $\begin{array}{l}\text { HG-PPM } \\
\mathrm{CV}\end{array}$ & $\begin{array}{l}\text { F\% } \\
\text { ISE }\end{array}$ \\
\hline $\begin{array}{l}\text { 7DH3195 } \\
7 D H 3196 A \\
7 D H 3196 B \\
7 D H 3198 \\
7 D H 3203\end{array}$ & $\begin{array}{l}N \\
N \\
<1.00 \\
1.00 \\
1.00\end{array}$ & $\begin{array}{l}N \\
.200 \\
.300 \\
N \\
N\end{array}$ & $\begin{array}{l}N \\
N \\
2.00 \\
6.00 \\
N\end{array}$ & $\begin{array}{c}N \\
<5.00 \\
50.00 \\
<5.00 \\
N\end{array}$ & $\begin{array}{l}\cdots \\
\cdots \\
\cdots \\
\cdots\end{array}$ & $\begin{array}{l}-- \\
-- \\
-- \\
--\end{array}$ & $\begin{array}{l}\cdots \\
\cdots \\
\cdots \\
\cdots \\
\cdots\end{array}$ & $\begin{array}{l}\cdots \\
\cdots \\
\cdots \\
\cdots\end{array}$ & $\begin{array}{l}\cdots \\
\cdots \\
\cdots \\
\cdots\end{array}$ & $\begin{array}{l}\cdots \\
\cdots \\
\cdots \\
\cdots\end{array}$ \\
\hline $\begin{array}{l}7 D H 3207 \\
7 D H 3212 \\
70 H 3214 \\
70 H 3215 A \\
7 D H 3215 B\end{array}$ & $\begin{array}{l}N \\
1.00 \\
N \\
2.00 \\
N\end{array}$ & $\begin{array}{l}N \\
N \\
N \\
.100\end{array}$ & $\begin{array}{c}2.00 \\
N \\
N \\
N \\
<2.00\end{array}$ & $\begin{array}{l}80.00 \\
50.00 \\
15.00 \\
50.00 \\
70.00\end{array}$ & $\begin{array}{l}-- \\
-- \\
\cdots \\
\cdots\end{array}$ & $\begin{array}{l}\cdots \\
\cdots \\
\cdots \\
\cdots\end{array}$ & $\begin{array}{l}-- \\
\cdots \\
\cdots \\
\cdots \\
\cdots\end{array}$ & $\begin{array}{l}-- \\
\cdots \\
-- \\
\cdots \\
\cdots\end{array}$ & $\begin{array}{l}-. \\
-- \\
\cdots \\
\cdots \\
--\end{array}$ & $\begin{array}{l}\cdots \\
\cdots \\
\cdots \\
\cdots \\
\cdots\end{array}$ \\
\hline $\begin{array}{l}70 H 3218 \\
7 D H 3219 A \\
7 D H 3219 B \\
7 D H 3219 C \\
7 D H 3220\end{array}$ & $\begin{array}{c}N \\
N \\
N \\
>1,000.00 \\
67.00\end{array}$ & $\begin{array}{r}.^{.100} \\
1.000 \\
>100.000 \\
.400\end{array}$ & $\begin{array}{r}N \\
N \\
94.00 \\
>1,000.00 \\
16.00\end{array}$ & $\begin{array}{r}55.00 \\
50.00 \\
290.00 \\
130.00 \\
5.00\end{array}$ & $\begin{array}{l}\cdots \\
\cdots \\
\cdots \\
\cdots\end{array}$ & $\begin{array}{l}-. \\
-- \\
\cdots \\
\cdots\end{array}$ & $\begin{array}{l}\cdots \\
\cdots \\
\cdots \\
\cdots\end{array}$ & $\begin{array}{l}-- \\
-- \\
\cdots \\
\cdots \\
--\end{array}$ & $\begin{array}{l}-. \\
\cdots \\
\cdots \\
\cdots\end{array}$ & $\begin{array}{l}- \\
-. \\
\cdots \\
\cdots \\
\cdots\end{array}$ \\
\hline $\begin{array}{l}70 H 3222 \\
70 H 3224 \\
70 H 3226 \\
70 H 3227 \\
7 D H 3228\end{array}$ & $\begin{array}{r}25.00 \\
<1.00 \\
5.00 \\
11.00 \\
2.00\end{array}$ & $\begin{array}{l}N \\
N \\
N \\
.100 \\
.100\end{array}$ & $\begin{array}{l}4.00 \\
N \\
N \\
2.00 \\
N\end{array}$ & $\begin{array}{c}N \\
100.00 \\
70.00 \\
70.00 \\
N\end{array}$ & $\begin{array}{l}-. \\
-- \\
-- \\
--\end{array}$ & $\begin{array}{l}-. \\
\cdots \\
\cdots \\
\cdots\end{array}$ & $\begin{array}{l}\ldots \\
\cdots \\
\cdots \\
\cdots \\
--\end{array}$ & $\begin{array}{l}- \\
\cdots \\
\cdots \\
\cdots \\
\cdots\end{array}$ & $\begin{array}{l}\cdots \\
\cdots \\
\cdots \\
\cdots \\
--\end{array}$ & $\begin{array}{l}\ldots \\
\ldots \\
\ldots \\
\cdots \\
\cdots\end{array}$ \\
\hline $\begin{array}{l}70 H 3229 \\
70 H 3231 \\
70 H 3249 \\
80 H 3276 A \\
80 H 3276 B\end{array}$ & $\begin{array}{r}6.00 \\
1.00 \\
2.00 \\
<2.00 \\
<2.00\end{array}$ & $\begin{array}{l}\dot{N}^{.100} \\
2.500 \\
<.100 \\
<.100\end{array}$ & $\begin{array}{c}N \\
N \\
8.00 \\
<2.00 \\
<2.00\end{array}$ & $\begin{array}{r}<5.00 \\
30.00 \\
530.00 \\
19.00 \\
6.00\end{array}$ & $\begin{array}{l}\cdots \\
\cdots \\
\cdots \\
N \\
N\end{array}$ & $\begin{array}{l}-- \\
\cdots \\
\cdots \\
N\end{array}$ & $\begin{array}{l}\ldots \\
\ldots \\
\ldots \\
<5 \\
<5\end{array}$ & $\begin{array}{l}\cdots \\
\cdots \\
-- \\
N \\
N\end{array}$ & $\begin{array}{l}\ldots \\
\cdots \\
\cdots \\
\mathrm{N} \\
.02\end{array}$ & $\begin{array}{l}\ldots \\
\ldots \\
-. \\
<.01 \\
<.01\end{array}$ \\
\hline $\begin{array}{l}80 H 3283 \\
80 H 3284 A \\
80 H 3284 B \\
80 H 3287 A \\
80 H 3287 B\end{array}$ & $\begin{array}{r}<2.00 \\
<2.00 \\
<2.00 \\
130.00 \\
<2.00\end{array}$ & $\begin{array}{r}<.100 \\
.300 \\
1.500 \\
17.000 \\
1.000\end{array}$ & $\begin{array}{r}<2.00 \\
<2.00 \\
2.00 \\
110.00 \\
<2.00\end{array}$ & $\begin{array}{r}<2.00 \\
42.00 \\
77.00 \\
5,200.00 \\
210.00\end{array}$ & $\begin{array}{l}N \\
N \\
N \\
N \\
N\end{array}$ & $\begin{array}{l}.2 \\
<.2 \\
N \\
K .2\end{array}$ & $\begin{array}{r}20 \\
10 \\
10 \\
10 \\
N\end{array}$ & $\begin{array}{l}N \\
N \\
N \\
N \\
N\end{array}$ & $\begin{array}{l}.10 \\
N \\
N \\
4.00 \\
1.36\end{array}$ & $\begin{array}{l}.04 \\
.04 \\
.02 \\
.01 \\
<.01\end{array}$ \\
\hline $\begin{array}{l}80 H 3288 B \\
80 H 3289 B \\
80 H 3291 \\
80 H 3292 B \\
80 H 3294 B\end{array}$ & $\begin{array}{r}130.00 \\
49.00 \\
<2.00 \\
<2.00 \\
3.00\end{array}$ & $\begin{array}{l}7.100 \\
2.400 \\
<.100 \\
.400 \\
.600\end{array}$ & $\begin{array}{r}110.00 \\
77.00 \\
<2.00 \\
<2.00 \\
<2.00\end{array}$ & $\begin{array}{r}160.00 \\
880.00 \\
9.00 \\
42.00 \\
25.00\end{array}$ & $\begin{array}{l}N \\
N \\
N \\
3.0 \\
3.0\end{array}$ & $\begin{array}{l}N \\
N \\
N \\
<.2 \\
<.2\end{array}$ & $\begin{array}{r}20 \\
15 \\
N \\
20 \\
20\end{array}$ & $\begin{array}{l}N \\
N \\
N \\
N \\
N\end{array}$ & $\begin{array}{l}5.20 \\
1.48 \\
N \\
N \\
.04\end{array}$ & $\begin{array}{l}.02 \\
.03 \\
.05 \\
.05 \\
.11\end{array}$ \\
\hline $\begin{array}{l}80 H 3294 \mathrm{C} \\
80 H 3295 \\
80 H 3296 \\
80 H 3298 \\
80 H 3299\end{array}$ & $\begin{array}{r}6.00 \\
<2.00 \\
<2.00 \\
<2.00 \\
7.00\end{array}$ & $\begin{array}{l}.400 \\
.400 \\
.100 \\
.700 \\
.300\end{array}$ & $\begin{array}{r}6.00 \\
<2.00 \\
<2.00 \\
<2.00 \\
<2.00\end{array}$ & $\begin{array}{r}49.00 \\
88.00 \\
3.00 \\
46.00 \\
4.00\end{array}$ & $\begin{array}{l}1.0 \\
1.0 \\
N \\
2.0 \\
N\end{array}$ & $\begin{array}{l}<.2 \\
<.2 \\
\mathrm{~N} \\
\mathrm{~N} \\
.2\end{array}$ & $\begin{array}{r}20 \\
30 \\
N \\
20 \\
10\end{array}$ & $\begin{array}{l}\mathbf{N} \\
\mathbf{N} \\
\mathbf{N} \\
\mathbf{N} \\
\mathbf{N}\end{array}$ & $\begin{array}{l}.04 \\
\mathrm{~N} \\
\mathrm{~N} \\
.04\end{array}$ & $\begin{array}{l}.04 \\
.07 \\
<.01 \\
.05 \\
.04\end{array}$ \\
\hline $\begin{array}{l}80 H 3300 \\
80 H 3302 A \\
80 H 3302 B \\
80 H 3303 \\
80 H 3305\end{array}$ & $\begin{array}{l}<2.00 \\
<2.00 \\
<2.00 \\
<2.00 \\
<2.00\end{array}$ & $\begin{array}{l}.400 \\
.300 \\
.200 \\
.600 \\
2.500\end{array}$ & $\begin{array}{r}<2.00 \\
2.00 \\
28.00 \\
43.00 \\
<2.00\end{array}$ & $\begin{array}{r}43.00 \\
14.00 \\
6.00 \\
9.00 \\
11.00\end{array}$ & $\begin{array}{l}N \\
N \\
.5 \\
N \\
1.0\end{array}$ & $\begin{array}{l}.2 \\
.3 \\
.2 \\
.5\end{array}$ & $\begin{array}{r}15 \\
7 \\
15 \\
10 \\
15\end{array}$ & $\begin{array}{l}N \\
N \\
N \\
N \\
N\end{array}$ & $\begin{array}{l}.06 \\
.04 \\
N \\
.10\end{array}$ & $\begin{array}{l}.03 \\
.01 \\
.03 \\
.01 \\
.03\end{array}$ \\
\hline
\end{tabular}


TABLE 4--RESULTS OF ANALYSES, DETROIT MINING DISTRICT, UTAH--Cont inued

\begin{tabular}{|c|c|c|c|c|c|c|c|c|c|c|c|}
\hline Sample & Latitude & Longi & i tude & $\begin{array}{c}\text { Fe-pct } \\
\text { s }\end{array}$ & $\begin{array}{c}\text { Mg-pct. } \\
\text { s }\end{array}$ & $\begin{array}{c}\text { Ca-pct. } \\
s\end{array}$ & $\underset{\mathbf{s}}{\mathrm{Ti}-\mathrm{pct} .}$ & $\begin{array}{c}\text { Mn-ppm } \\
s\end{array}$ & $\begin{array}{c}\text { Ag-ppm } \\
\mathrm{s}\end{array}$ & $\begin{array}{c}\text { As-ppm } \\
s\end{array}$ & $\begin{array}{c}\text { AU-ppm } \\
\text { s }\end{array}$ \\
\hline $\begin{array}{l}80 H 3306 \\
80 H 3307 A \\
80 H 3307 B \\
80 H 3319 A \\
80 H 3319 B\end{array}$ & $\begin{array}{lll}39 & 32 & 23 \\
39 & 32 & 21 \\
39 & 32 & 21 \\
39 & 32 & 14 \\
39 & 32 & 14\end{array}$ & $\begin{array}{l}1125 \\
1125 \\
1125 \\
1125 \\
1125\end{array}$ & $\begin{array}{ll}59 & 25 \\
59 & 31 \\
59 & 31 \\
59 & 47 \\
59 & 47\end{array}$ & $\begin{array}{l}1.50 \\
5.00 \\
5.00 \\
2.00 \\
1.00\end{array}$ & $\begin{array}{r}.05 \\
.20 \\
.10 \\
<.02 \\
<.02\end{array}$ & $\begin{array}{l}.10 \\
.10 \\
.20 \\
.07 \\
.50\end{array}$ & $\begin{array}{l}.150 \\
.500 \\
.150 \\
.150 \\
.100\end{array}$ & $\begin{array}{r}50 \\
10 \\
50 \\
100 \\
10\end{array}$ & $\begin{array}{l}N \\
N \\
N \\
N \\
N\end{array}$ & $\begin{array}{l}N \\
N \\
N \\
N \\
N\end{array}$ & $\begin{array}{l}\mathbf{N} \\
\mathbf{N} \\
\mathbf{N} \\
\mathbf{N} \\
\mathbf{N}\end{array}$ \\
\hline $\begin{array}{l}80 H 3322 \\
8013323 \\
80 H 3325 A \\
80 H 3325 B \\
80 H 3325 C\end{array}$ & $\begin{array}{lll}39 & 31 & 43 \\
39 & 31 & 40 \\
39 & 33 & 42 \\
39 & 33 & 42 \\
39 & 33 & 42\end{array}$ & $\begin{array}{l}113 \\
1125 \\
113 \\
113 \\
113\end{array}$ & $\begin{array}{rr}0 & 2 \\
59 & 58 \\
0 & 42 \\
0 & 42 \\
0 & 42\end{array}$ & $\begin{array}{r}7.00 \\
10.00 \\
5.00 \\
5.00 \\
1.00\end{array}$ & $\begin{array}{r}.10 \\
.15 \\
1.00 \\
.10 \\
<.02\end{array}$ & $\begin{array}{r}.10 \\
.10 \\
<.05 \\
.07 \\
<.05\end{array}$ & $\begin{array}{l}.100 \\
.007 \\
.500 \\
.050 \\
.030\end{array}$ & $\begin{array}{r}70 \\
100 \\
100 \\
30 \\
20\end{array}$ & $\begin{array}{c}N \\
N \\
1.00 \\
30.00 \\
10.00\end{array}$ & $\begin{array}{l}N \\
N \\
N \\
N \\
N\end{array}$ & $\begin{array}{l}\mathbf{N} \\
\mathbf{N} \\
\mathbf{N} \\
\mathbf{N} \\
\mathbf{N}\end{array}$ \\
\hline $\begin{array}{l}80 H 3325 D \\
80 H 3326 A \\
80 H 3326 B \\
80 H 3326 C \\
80 H 3327 A\end{array}$ & $\begin{array}{lll}39 & 33 & 42 \\
39 & 30 & 59 \\
39 & 30 & 59 \\
39 & 30 & 59 \\
39 & 32 & 43\end{array}$ & $\begin{array}{l}113 \\
113 \\
113 \\
113 \\
113\end{array}$ & $\begin{array}{ll}0 & 42 \\
1 & 47 \\
1 & 47 \\
1 & 47 \\
3 & 18\end{array}$ & $\begin{array}{l}3.00 \\
1.00 \\
1.00 \\
.30 \\
.50\end{array}$ & $\begin{array}{r}<.02 \\
<.02 \\
.05 \\
7.00 \\
.15\end{array}$ & $\begin{array}{r}.05 \\
<.05 \\
<.05 \\
>20.00 \\
1.00\end{array}$ & $\begin{array}{l}.200 \\
.200 \\
.300 \\
.010 \\
.005\end{array}$ & $\begin{array}{r}30 \\
100 \\
100 \\
70 \\
100\end{array}$ & $\begin{array}{l}N \\
7.00 \\
5.00 \\
N \\
N\end{array}$ & $\begin{array}{l}\mathbf{N} \\
\mathbf{N} \\
\mathbf{N} \\
\mathbf{N} \\
\mathbf{N}\end{array}$ & $\begin{array}{r}N \\
15 \\
<10 \\
N \\
N\end{array}$ \\
\hline $\begin{array}{l}80 H 3327 B \\
8 D H 3327 C \\
80 H 33270 \\
80 H 3328 A \\
80 H 3328 B\end{array}$ & $\begin{array}{lll}39 & 32 & 43 \\
39 & 32 & 43 \\
39 & 32 & 43 \\
39 & 33 & 32 \\
39 & 33 & 32\end{array}$ & $\begin{array}{l}113 \\
113 \\
113 \\
113 \\
113\end{array}$ & $\begin{array}{ll}3 & 18 \\
3 & 18 \\
3 & 18 \\
3 & 28 \\
3 & 28\end{array}$ & $\begin{array}{r}.10 \\
7.00 \\
5.00 \\
>20.00 \\
>20.00\end{array}$ & $\begin{array}{r}10.00 \\
.70 \\
1.00 \\
.10 \\
.10\end{array}$ & $\begin{array}{r}20.00 \\
.05 \\
.05 \\
.05 \\
.05\end{array}$ & $\begin{array}{l}.002 \\
.200 \\
.200 \\
.050 \\
.050\end{array}$ & $\begin{array}{r}150 \\
30 \\
20 \\
200 \\
150\end{array}$ & $\begin{array}{l}\mathrm{N} \\
\mathrm{N} \\
\mathrm{N} \\
\mathrm{N} \\
\mathrm{N}\end{array}$ & $\begin{array}{l}\mathbf{N} \\
\mathbf{N} \\
\mathbf{N} \\
\mathbf{N} \\
\mathbf{N}\end{array}$ & $\begin{array}{l}N \\
N \\
N \\
N \\
N\end{array}$ \\
\hline $\begin{array}{l}80 H 3328 C \\
80 H 33280 \\
80 H 3328 E \\
80 H 3329 A \\
80 H 3329 B\end{array}$ & $\begin{array}{lll}39 & 33 & 32 \\
39 & 33 & 32 \\
39 & 33 & 32 \\
39 & 33 & 47 \\
39 & 33 & 47\end{array}$ & $\begin{array}{l}113 \\
113 \\
113 \\
113 \\
113\end{array}$ & $\begin{array}{ll}3 & 28 \\
3 & 28 \\
3 & 28 \\
3 & 17 \\
3 & 17\end{array}$ & $\begin{array}{r}5.00 \\
.30 \\
>20.00 \\
1.00 \\
1.00\end{array}$ & $\begin{array}{r}.30 \\
7.00 \\
.20 \\
<.02 \\
.05\end{array}$ & $\begin{array}{r}20.00 \\
>20.00 \\
.10 \\
.07 \\
.20\end{array}$ & $\begin{array}{r}.200 \\
<.002 \\
.020 \\
.150 \\
.300\end{array}$ & $\begin{array}{r}700 \\
100 \\
200 \\
50 \\
300\end{array}$ & $\begin{array}{c}N \\
N \\
N \\
10.00 \\
700.00\end{array}$ & $\begin{array}{r}N \\
N \\
N \\
500 \\
5,000\end{array}$ & $\begin{array}{r}N \\
N \\
N \\
N \\
20\end{array}$ \\
\hline $\begin{array}{l}80 H 3329 C \\
80 H 3330 A \\
80 H 3330 B \\
80 H 3330 C \\
80 H 3330 D\end{array}$ & $\begin{array}{lll}39 & 33 & 47 \\
39 & 33 & 49 \\
39 & 33 & 49 \\
39 & 33 & 49 \\
39 & 33 & 49\end{array}$ & $\begin{array}{l}113 \\
113 \\
113 \\
113 \\
113\end{array}$ & $\begin{array}{ll}3 & 17 \\
3 & 13 \\
3 & 13 \\
3 & 13 \\
3 & 13\end{array}$ & $\begin{array}{r}.15 \\
1.00 \\
5.00 \\
>20.00 \\
3.00\end{array}$ & $\begin{array}{r}1.50 \\
.02 \\
.20 \\
.30 \\
.10\end{array}$ & $\begin{array}{r}>20.00 \\
.20 \\
.30 \\
.20 \\
.20\end{array}$ & $\begin{array}{l}.005 \\
.070 \\
.070 \\
.100 \\
.050\end{array}$ & $\begin{array}{r}3,000 \\
70 \\
50 \\
70 \\
50\end{array}$ & $\begin{array}{r}30.00 \\
<.50 \\
1.00 \\
.50 \\
1.00\end{array}$ & $\begin{array}{l}\mathbf{N} \\
\mathbf{N} \\
\mathbf{N} \\
\mathbf{N} \\
\mathbf{N}\end{array}$ & $\begin{array}{l}\mathrm{N} \\
\mathrm{N} \\
\mathrm{N} \\
\mathrm{N} \\
\mathrm{N}\end{array}$ \\
\hline $\begin{array}{l}80 H 3330 E \\
80 H 3331 A \\
80 H 3331 B \\
80 H 3331 C \\
80 H 3331 D\end{array}$ & $\begin{array}{lll}39 & 33 & 49 \\
39 & 33 & 57 \\
39 & 33 & 57 \\
39 & 33 & 57 \\
39 & 33 & 57\end{array}$ & $\begin{array}{l}113 \\
113 \\
113 \\
113 \\
113\end{array}$ & $\begin{array}{ll}3 & 13 \\
3 & 18 \\
3 & 18 \\
3 & 18 \\
3 & 18\end{array}$ & $\begin{array}{r}.10 \\
>20.00 \\
15.00 \\
.70 \\
7.00\end{array}$ & $\begin{array}{l}.03 \\
.07 \\
.05 \\
.50 \\
.05\end{array}$ & $\begin{array}{r}.10 \\
.07 \\
.07 \\
>20.00 \\
.20\end{array}$ & $\begin{array}{l}.200 \\
.015 \\
.050 \\
.002 \\
.050\end{array}$ & $\begin{array}{r}<10 \\
50 \\
50 \\
2,000 \\
150\end{array}$ & $\begin{array}{l}N \\
7.00 \\
3.00 \\
N \\
3.00\end{array}$ & $\begin{array}{r}N \\
300 \\
200 \\
N \\
N\end{array}$ & $\begin{array}{l}\mathbf{N} \\
\mathbf{N} \\
\mathbf{N} \\
\mathbf{N} \\
\mathbf{N}\end{array}$ \\
\hline $\begin{array}{l}80 H 3332 A \\
80 H 3332 B \\
80 H 3332 C \\
80 H 3332 D \\
80 H 3332 E\end{array}$ & $\begin{array}{lll}39 & 33 & 56 \\
39 & 33 & 56 \\
39 & 33 & 56 \\
39 & 33 & 56 \\
39 & 33 & 56\end{array}$ & $\begin{array}{l}113 \\
113 \\
113 \\
113 \\
113\end{array}$ & $\begin{array}{ll}3 & 1 \\
3 & 1 \\
3 & 1 \\
3 & 1 \\
3 & 1\end{array}$ & $\begin{array}{r}.20 \\
.05 \\
2.00 \\
>20.00 \\
10.00\end{array}$ & $\begin{array}{r}2.00 \\
>10.00 \\
2.00 \\
.10 \\
.20\end{array}$ & $\begin{array}{r}>20.00 \\
20.00 \\
7.00 \\
.50 \\
>20.00\end{array}$ & $\begin{array}{r}.010 \\
<.002 \\
.500 \\
.100 \\
.070\end{array}$ & $\begin{array}{r}70 \\
50 \\
100 \\
50 \\
1,000\end{array}$ & $\begin{array}{c}N \\
N \\
5.00 \\
1,500.00 \\
50.00\end{array}$ & $\begin{array}{r}N \\
N \\
N \\
7,000 \\
1,500\end{array}$ & $\begin{array}{l}N \\
N \\
N \\
N \\
N\end{array}$ \\
\hline $\begin{array}{l}80 H 3332 F \\
80 H 3333 \\
80 H 3334 A \\
80 H 3334 B \\
80 H 3336\end{array}$ & $\begin{array}{rrr}39 & 33 & 56 \\
39 & 34 & 2 \\
39 & 34 & 7 \\
39 & 34 & 7 \\
39 & 31 & 50\end{array}$ & $\begin{array}{l}113 \\
113 \\
113 \\
113 \\
113\end{array}$ & $\begin{array}{ll}3 & 1 \\
3 & 5 \\
3 & 4 \\
3 & 4 \\
0 & 0\end{array}$ & $\begin{array}{r}.50 \\
7.00 \\
.70 \\
10.00 \\
.50\end{array}$ & $\begin{array}{l}<.02 \\
<.02 \\
<.02 \\
.20 \\
<.02\end{array}$ & $\begin{array}{l}.05 \\
.07 \\
.10 \\
.10 \\
.10\end{array}$ & $\begin{array}{l}.200 \\
.050 \\
.100 \\
.150 \\
.100\end{array}$ & $\begin{array}{r}70 \\
70 \\
200 \\
100 \\
100\end{array}$ & $\begin{array}{l}N \\
3.00 \\
3.00 \\
1.00 \\
N\end{array}$ & $\begin{array}{r}N \\
N \\
N \\
500 \\
N\end{array}$ & $\begin{array}{l}N \\
N \\
N \\
N \\
N\end{array}$ \\
\hline
\end{tabular}


TABLE 4--RESULTS OF ANALYSES, DETROIT MINING DISTRICT, UTAH--Continued

\begin{tabular}{|c|c|c|c|c|c|c|c|c|c|c|c|c|c|}
\hline Sample & $\begin{array}{c}\text { B-ppm } \\
\text { s }\end{array}$ & $\begin{array}{c}\text { Ba-ppm } \\
\mathrm{s}\end{array}$ & $\begin{array}{c}\text { Be-ppm } \\
\text { s }\end{array}$ & $\begin{array}{c}\text { Bi-ppm } \\
s\end{array}$ & $\underset{s}{\text { Cd-ppm }}$ & $\begin{array}{l}\text { Co-ppm } \\
\text { s }\end{array}$ & $\begin{array}{c}\mathrm{Cr}-\mathrm{ppm} \\
\mathrm{s}\end{array}$ & $\begin{array}{l}\text { Cu-ppm } \\
s\end{array}$ & $\begin{array}{l}\text { La-ppm } \\
s\end{array}$ & $\begin{array}{c}\text { Mo-ppm } \\
\mathrm{s}\end{array}$ & $\begin{array}{l}\text { Nb-ppm } \\
s\end{array}$ & $\underset{s}{\mathrm{Ni}-\mathrm{ppm}}$ & $\begin{array}{c}\mathrm{Pb}-\mathrm{ppm} \\
\mathrm{s}\end{array}$ \\
\hline $\begin{array}{l}80 H 3306 \\
80 H 3307 A \\
80 H 3307 B \\
80 H 3319 A \\
80 H 3319 B\end{array}$ & $\begin{array}{l}<10 \\
<10 \\
<10 \\
200 \\
<10\end{array}$ & $\begin{array}{l}200 \\
300 \\
500 \\
200 \\
200\end{array}$ & $\begin{array}{l}\mathbf{N} \\
\mathbf{N} \\
\mathbf{N} \\
\mathbf{N} \\
\mathbf{N}\end{array}$ & $\begin{array}{l}N \\
N \\
N \\
N \\
N\end{array}$ & $\begin{array}{l}N \\
N \\
N \\
N \\
N\end{array}$ & $\begin{array}{r}N \\
10 \\
<10 \\
N \\
N\end{array}$ & $\begin{array}{r}<10 \\
50 \\
20 \\
<10 \\
<10\end{array}$ & $\begin{array}{r}7 \\
15 \\
15 \\
7 \\
<5\end{array}$ & $\begin{array}{r}N \\
50 \\
<50 \\
N \\
N\end{array}$ & $\begin{array}{r}N \\
N \\
N \\
10 \\
N\end{array}$ & $\begin{array}{l}\mathbf{N} \\
\mathbf{N} \\
\mathbf{N} \\
\mathbf{N} \\
\mathbf{N}\end{array}$ & $\begin{array}{r}<5 \\
50 \\
15 \\
7 \\
<5\end{array}$ & $\begin{array}{r}<10 \\
20 \\
50 \\
10 \\
10\end{array}$ \\
\hline $\begin{array}{l}80 H 3322 \\
8013323 \\
80 H 3325 A \\
80 H 3325 B \\
80 H 3325 C\end{array}$ & $\begin{array}{r}20 \\
10 \\
20 \\
20 \\
<10\end{array}$ & $\begin{array}{l}200 \\
300 \\
200 \\
150 \\
300\end{array}$ & $\begin{array}{c}<1.0 \\
<1.0 \\
N \\
<1.0 \\
N\end{array}$ & $\begin{array}{r}N \\
N \\
N \\
10 \\
15\end{array}$ & $\begin{array}{l}N \\
N \\
N \\
N \\
N\end{array}$ & $\begin{array}{l}N \\
N \\
N \\
N \\
N\end{array}$ & $\begin{array}{r}10 \\
15 \\
<10 \\
N \\
N\end{array}$ & $\begin{array}{r}7 \\
5 \\
100 \\
300 \\
700\end{array}$ & $\begin{array}{l}N \\
N \\
N \\
N \\
N\end{array}$ & $\begin{array}{r}N \\
N \\
5 \\
<5 \\
5\end{array}$ & $\begin{array}{l}N \\
N \\
N \\
N \\
N\end{array}$ & $\begin{array}{r}5 \\
7 \\
N \\
<5 \\
10\end{array}$ & $\begin{array}{l}<10 \\
<10 \\
200 \\
700 \\
500\end{array}$ \\
\hline $\begin{array}{l}80 H 3325 D \\
80 H 3326 A \\
80 H 3326 B \\
80 H 3326 C \\
80 H 3327 A\end{array}$ & $\begin{array}{r}10 \\
<10 \\
<10 \\
N \\
10\end{array}$ & $\begin{array}{l}200 \\
500 \\
100 \\
300 \\
200\end{array}$ & $\begin{array}{r}<1.0 \\
<1.0 \\
<1.0 \\
\quad N \\
<1.0\end{array}$ & $\begin{array}{l}N \\
N \\
N \\
N \\
N\end{array}$ & $\begin{array}{l}\text { N } \\
N \\
N \\
N \\
N\end{array}$ & $\begin{array}{l}N \\
N \\
N \\
N \\
N\end{array}$ & $\begin{array}{r}20 \\
N \\
10 \\
N \\
N\end{array}$ & $\begin{array}{l}5 \\
20 \\
20 \\
<5 \\
30\end{array}$ & $\begin{array}{r}<50 \\
N \\
N \\
N \\
N\end{array}$ & $\begin{array}{r}N \\
30 \\
7 \\
N \\
N\end{array}$ & $\begin{array}{l}N \\
N \\
N \\
N \\
N\end{array}$ & $\begin{array}{r}5 \\
7 \\
7 \\
<5 \\
10\end{array}$ & $\begin{array}{r}20 \\
500 \\
30 \\
<10 \\
<10\end{array}$ \\
\hline $\begin{array}{l}80 H 3327 B \\
80 H 3327 C \\
80 H 33270 \\
80 H 3328 A \\
80 H 3328 B\end{array}$ & $\begin{array}{r}30 \\
20 \\
20 \\
<10 \\
<10\end{array}$ & $\begin{array}{r}20 \\
200 \\
200 \\
50 \\
50\end{array}$ & $\begin{array}{r}N \\
1.0 \\
1.0 \\
<1.0 \\
<1.0\end{array}$ & $\begin{array}{l}N \\
N \\
N \\
N \\
N\end{array}$ & $\begin{array}{l}N \\
N \\
N \\
N \\
N\end{array}$ & $\begin{array}{r}N \\
<10 \\
N \\
30 \\
20\end{array}$ & $\begin{array}{r}N \\
50 \\
70 \\
N \\
<10\end{array}$ & $\begin{array}{r}5 \\
100 \\
100 \\
30 \\
20\end{array}$ & $\begin{array}{r}N \\
<50 \\
50 \\
N \\
N\end{array}$ & $\begin{array}{l}\text { N } \\
7 \\
5 \\
N \\
N\end{array}$ & $\begin{array}{l}N \\
N \\
N \\
N \\
N\end{array}$ & $\begin{array}{l}<5 \\
70 \\
70 \\
50 \\
20\end{array}$ & $\begin{array}{r}N \\
10 \\
10 \\
<10 \\
<10\end{array}$ \\
\hline $\begin{array}{l}80 H 3328 C \\
80 H 33280 \\
80 H 3328 E \\
80 H 3329 A \\
80 H 3329 B\end{array}$ & $\begin{array}{r}10 \\
N \\
50 \\
10 \\
10\end{array}$ & $\begin{array}{r}30 \\
<20 \\
70 \\
50 \\
1,000\end{array}$ & $\begin{array}{c}1.0 \\
N \\
3.0 \\
N \\
<1.0\end{array}$ & $\begin{array}{r}N \\
N \\
N \\
>1,000 \\
>1,000\end{array}$ & $\begin{array}{l}\mathrm{N} \\
\mathrm{N} \\
\mathrm{N} \\
\mathrm{N} \\
\mathrm{N}\end{array}$ & $\begin{array}{r}20 \\
N \\
50 \\
<10 \\
<10\end{array}$ & $\begin{array}{r}10 \\
N \\
50 \\
<10 \\
15\end{array}$ & $\begin{array}{r}7 \\
<5 \\
100 \\
5,000 \\
>20,000\end{array}$ & $\begin{array}{r}50 \\
N \\
N \\
N \\
<50\end{array}$ & $\begin{array}{r}10 \\
N \\
N \\
N \\
N\end{array}$ & $\begin{array}{r}N \\
N \\
N \\
N \\
<20\end{array}$ & $\begin{array}{r}20 \\
N \\
100 \\
5 \\
15\end{array}$ & $\begin{array}{r}10 \\
<10 \\
N \\
50 \\
1,000\end{array}$ \\
\hline $\begin{array}{l}80 H 3329 C \\
80 H 3330 A \\
80 H 3330 B \\
80 H 3330 C \\
80 H 3330 D\end{array}$ & $\begin{array}{r}N \\
10 \\
10 \\
<10 \\
10\end{array}$ & $\begin{array}{r}200 \\
300 \\
1,500 \\
1,500 \\
20\end{array}$ & $\begin{array}{c}N \\
N \\
N \\
N \\
<1.0\end{array}$ & $\begin{array}{l}N \\
N \\
N \\
N \\
N\end{array}$ & $\begin{array}{l}\text { N } \\
\text { N } \\
N \\
N \\
N\end{array}$ & $\begin{array}{r}10 \\
N \\
N \\
20 \\
<10\end{array}$ & $\begin{array}{r}N \\
<10 \\
15 \\
20 \\
<10\end{array}$ & $\begin{array}{r}500 \\
30 \\
30 \\
70 \\
50\end{array}$ & $\begin{array}{r}N \\
N \\
<50 \\
N \\
N\end{array}$ & $\begin{array}{l}N \\
N \\
N \\
7 \\
N\end{array}$ & $\begin{array}{l}\text { N } \\
N \\
N \\
N \\
N\end{array}$ & $\begin{array}{r}<5 \\
5 \\
5 \\
15 \\
10\end{array}$ & $\begin{array}{r}700 \\
200 \\
500 \\
3,000 \\
100\end{array}$ \\
\hline $\begin{array}{l}80 H 3330 E \\
80 H 3331 A \\
80 H 3331 B \\
80 H 3331 C \\
80 H 3331 D\end{array}$ & $\begin{array}{r}<10 \\
<10 \\
N \\
N \\
10\end{array}$ & $\begin{array}{r}1,500 \\
2,000 \\
700 \\
100 \\
200\end{array}$ & $\begin{array}{c}N \\
<1.0 \\
2.0 \\
N \\
N\end{array}$ & $\begin{array}{r}N \\
N \\
N \\
N \\
50\end{array}$ & $\begin{array}{l}\mathrm{N} \\
\mathrm{N} \\
\mathrm{N} \\
\mathrm{N} \\
\mathrm{N}\end{array}$ & $\begin{array}{r}N \\
15 \\
<10 \\
N \\
N\end{array}$ & $\begin{array}{r}50 \\
<10 \\
20 \\
<10 \\
<10\end{array}$ & $\begin{array}{r}50 \\
150 \\
>20,000 \\
100 \\
100\end{array}$ & $\begin{array}{r}50 \\
N \\
<50 \\
N \\
N\end{array}$ & $\begin{array}{l}N \\
N \\
N \\
N \\
N\end{array}$ & $\begin{array}{l}\text { N } \\
N \\
N \\
N \\
N\end{array}$ & $\begin{array}{r}<5 \\
50 \\
30 \\
N \\
10\end{array}$ & $\begin{array}{r}1,000 \\
700 \\
500 \\
500 \\
300\end{array}$ \\
\hline $\begin{array}{l}80 H 3332 A \\
80 H 3332 B \\
80 H 3332 C \\
80 H 3332 D \\
80 H 3332 E\end{array}$ & $\begin{array}{r}N \\
N \\
100 \\
N \\
N\end{array}$ & $\begin{array}{r}20 \\
N \\
200 \\
1,000 \\
50\end{array}$ & $\begin{array}{c}N \\
N \\
<1.0 \\
2.0 \\
N\end{array}$ & $\begin{array}{r}N \\
N \\
30 \\
>1,000 \\
>1,000\end{array}$ & $\begin{array}{l}N \\
N \\
N \\
N \\
N\end{array}$ & $\begin{array}{r}N \\
N \\
<10 \\
50 \\
30\end{array}$ & $\begin{array}{r}<10 \\
<10 \\
70 \\
20 \\
<10\end{array}$ & $\begin{array}{r}7 \\
10 \\
500 \\
>20,000 \\
7,000\end{array}$ & $\begin{array}{r}N \\
N \\
50 \\
<50 \\
N\end{array}$ & $\begin{array}{l}N \\
N \\
N \\
N \\
N\end{array}$ & $\begin{array}{l}N \\
N \\
N \\
N \\
N\end{array}$ & $\begin{array}{r}N \\
N \\
15 \\
100 \\
30\end{array}$ & $\begin{array}{r}10 \\
15 \\
200 \\
1,000 \\
2,000\end{array}$ \\
\hline $\begin{array}{l}80 H 3332 F \\
80 H 3333 \\
80 H 3334 A \\
80 H 3334 B \\
80 H 3336\end{array}$ & $\begin{array}{r}10 \\
10 \\
15 \\
<10 \\
10\end{array}$ & $\begin{array}{r}50 \\
1,000 \\
1,500 \\
200 \\
70\end{array}$ & $\begin{array}{c}N \\
<1.0 \\
2.0 \\
2.0 \\
N\end{array}$ & $\begin{array}{r}N \\
<10 \\
N \\
N \\
N\end{array}$ & $\begin{array}{l}N \\
N \\
N \\
N \\
N\end{array}$ & $\begin{array}{r}<10 \\
N \\
N \\
N \\
N\end{array}$ & $\begin{array}{r}20 \\
<10 \\
10 \\
70 \\
<10\end{array}$ & $\begin{array}{l}70 \\
70 \\
20 \\
70 \\
10\end{array}$ & $\begin{array}{r}N \\
N \\
N \\
N 50 \\
N\end{array}$ & $\begin{array}{r}N \\
N \\
N \\
10 \\
N\end{array}$ & $\begin{array}{l}N \\
N \\
N \\
N \\
N\end{array}$ & $\begin{array}{r}5 \\
7 \\
5 \\
10 \\
5\end{array}$ & $\begin{array}{r}<10 \\
50 \\
20 \\
500 \\
30\end{array}$ \\
\hline
\end{tabular}


TABLE 4--RESULTS OF ANALYSES, DETROIT MINING DISTRICT, UTAH--Cont inued

\begin{tabular}{|c|c|c|c|c|c|c|c|c|c|c|c|c|}
\hline Sample & $\begin{array}{c}\text { sb-ppm } \\
s\end{array}$ & $\begin{array}{c}\text { Sc-ppm } \\
s\end{array}$ & $\begin{array}{c}\text { Sn-ppm } \\
s\end{array}$ & $\begin{array}{c}\text { Sr-ppm } \\
\text { s }\end{array}$ & $\begin{array}{c}v-p p m \\
s\end{array}$ & $\begin{array}{c}\text { W-ppm } \\
\text { s }\end{array}$ & $\begin{array}{c}Y-p p m \\
s\end{array}$ & $\begin{array}{c}\text { Zn-ppm } \\
s\end{array}$ & $\begin{array}{c}2 r-p p m \\
s\end{array}$ & $\begin{array}{c}\text { Th-ppm } \\
s\end{array}$ & $\begin{array}{c}\text { Au-ppm } \\
\text { aa }\end{array}$ & $\begin{array}{l}\text { AS-PPM } \\
\text { ICP }\end{array}$ \\
\hline $\begin{array}{l}80 H 3306 \\
80 H 3307 A \\
80 H 3307 B \\
80 H 3319 A \\
80 H 3319 B\end{array}$ & $\begin{array}{l}\mathbf{N} \\
\mathbf{N} \\
\mathbf{N} \\
\mathbf{N} \\
\mathbf{N}\end{array}$ & $\begin{array}{l}N \\
7 \\
5 \\
N \\
N\end{array}$ & $\begin{array}{l}N \\
N \\
N \\
N \\
N\end{array}$ & $\begin{array}{r}300 \\
500 \\
700 \\
N \\
200\end{array}$ & $\begin{array}{r}15 \\
200 \\
70 \\
20 \\
20\end{array}$ & $\begin{array}{l}N \\
N \\
N \\
N \\
N\end{array}$ & $\begin{array}{r}<10 \\
15 \\
10 \\
N \\
N\end{array}$ & $\begin{array}{l}N \\
N \\
N \\
N \\
N\end{array}$ & $\begin{array}{l}200 \\
200 \\
200 \\
150 \\
200\end{array}$ & $\begin{array}{l}N \\
N \\
N \\
N \\
N\end{array}$ & $\begin{array}{l}<.05 \\
<.05 \\
<.05 \\
<.05 \\
<.05\end{array}$ & $\begin{array}{r}100.0 \\
57.0 \\
61.0 \\
40.0 \\
6.0\end{array}$ \\
\hline $\begin{array}{l}80 H 3322 \\
8013323 \\
80 H 3325 A \\
80 H 3325 B \\
80 H 3325 C\end{array}$ & $\begin{array}{r}N \\
N \\
N \\
500 \\
500\end{array}$ & $\begin{array}{r}10 \\
7 \\
15 \\
N \\
N\end{array}$ & $\begin{array}{r}N \\
N \\
N \\
50 \\
70\end{array}$ & $\begin{array}{l}\mathbf{N} \\
\mathbf{N} \\
\mathbf{N} \\
\mathbf{N} \\
\mathbf{N}\end{array}$ & $\begin{array}{r}50 \\
50 \\
150 \\
15 \\
10\end{array}$ & $\begin{array}{l}\mathbf{N} \\
\mathbf{N} \\
\mathbf{N} \\
\mathbf{N} \\
\mathbf{N}\end{array}$ & $\begin{array}{r}70 \\
70 \\
200 \\
N \\
N\end{array}$ & $\begin{array}{l}N \\
N \\
N \\
N \\
N\end{array}$ & $\begin{array}{l}200 \\
300 \\
150 \\
100 \\
100\end{array}$ & $\begin{array}{l}N \\
N \\
N \\
N \\
N\end{array}$ & $\begin{array}{l}<.05 \\
<.05 \\
<.05 \\
.05 \\
.80\end{array}$ & $\begin{array}{r}<5.0 \\
<5.0 \\
82.0 \\
140.0 \\
280.0\end{array}$ \\
\hline $\begin{array}{l}80 H 3325 D \\
80 H 3326 A \\
80 H 3326 B \\
80 H 3326 C \\
80 H 3327 A\end{array}$ & $\begin{array}{r}N \\
200 \\
N \\
N \\
N\end{array}$ & $\begin{array}{l}N \\
N \\
N \\
N \\
N\end{array}$ & $\begin{array}{l}N \\
N \\
N \\
N \\
N\end{array}$ & $\begin{array}{r}100 \\
N \\
N \\
200 \\
N\end{array}$ & $\begin{array}{l}30 \\
15 \\
20 \\
10 \\
10\end{array}$ & $\begin{array}{l}N \\
N \\
N \\
N \\
N\end{array}$ & $\begin{array}{r}N \\
N \\
10 \\
N \\
N\end{array}$ & $\begin{array}{l}\mathbf{N} \\
\mathbf{N} \\
\mathbf{N} \\
\mathbf{N} \\
\mathbf{N}\end{array}$ & $\begin{array}{r}200 \\
50 \\
50 \\
<10 \\
<10\end{array}$ & $\begin{array}{l}N \\
N \\
N \\
N \\
N\end{array}$ & $\begin{array}{l}<.05 \\
8.60 \\
4.20 \\
.05 \\
<.05\end{array}$ & $\begin{array}{r}<5.0 \\
130.0 \\
36.0 \\
7.0 \\
21.0\end{array}$ \\
\hline $\begin{array}{l}80 H 3327 B \\
80 H 3327 C \\
80 H 33270 \\
80 H 3328 A \\
80 H 3328 B\end{array}$ & $\begin{array}{l}N \\
N \\
N \\
N \\
N\end{array}$ & $\begin{array}{r}N \\
10 \\
7 \\
N \\
N\end{array}$ & $\begin{array}{l}N \\
N \\
N \\
N \\
N\end{array}$ & $\begin{array}{l}\mathbf{N} \\
\mathbf{N} \\
\mathbf{N} \\
\mathbf{N} \\
\mathbf{N}\end{array}$ & $\begin{array}{r}<10 \\
50 \\
50 \\
15 \\
15\end{array}$ & $\begin{array}{l}\mathbf{N} \\
\mathbf{N} \\
\mathrm{N} \\
\mathrm{N} \\
\mathrm{N}\end{array}$ & $\begin{array}{r}N \\
50 \\
50 \\
10 \\
15\end{array}$ & $\begin{array}{r}N \\
N \\
N \\
500 \\
300\end{array}$ & $\begin{array}{r}N \\
200 \\
150 \\
20 \\
30\end{array}$ & $\begin{array}{l}\mathbf{N} \\
\mathbf{N} \\
\mathbf{N} \\
\mathbf{N} \\
\mathbf{N}\end{array}$ & $\begin{array}{l}<.05 \\
<.05 \\
<.05 \\
<.05 \\
<.05\end{array}$ & $\begin{array}{r}16.0 \\
16.0 \\
15.0 \\
<5.0 \\
6.0\end{array}$ \\
\hline $\begin{array}{l}80 H 3328 C \\
80 H 33280 \\
80 H 3328 E \\
80 H 3329 A \\
80 H 3329 B\end{array}$ & $\begin{array}{r}N \\
N \\
N \\
700 \\
>10,000\end{array}$ & $\begin{array}{r}5 \\
N \\
<5 \\
N \\
N\end{array}$ & $\begin{array}{r}N \\
N \\
N \\
50 \\
>1,000\end{array}$ & $\begin{array}{r}<100 \\
200 \\
N \\
N \\
100\end{array}$ & $\begin{array}{r}15 \\
<10 \\
15 \\
10 \\
30\end{array}$ & $\begin{array}{l}\mathbf{N} \\
\mathbf{N} \\
\mathbf{N} \\
\mathbf{N} \\
\mathbf{N}\end{array}$ & $\begin{array}{r}70 \\
N \\
<10 \\
10 \\
70\end{array}$ & $\begin{array}{r}N \\
N \\
500 \\
N \\
N\end{array}$ & $\begin{array}{r}200 \\
N \\
<10 \\
70 \\
100\end{array}$ & $\begin{array}{l}\mathbf{N} \\
\mathbf{N} \\
\mathbf{N} \\
\mathbf{N} \\
\mathbf{N}\end{array}$ & $\begin{array}{l}<.05 \\
<.05 \\
<.05 \\
2.50 \\
7.30\end{array}$ & $\begin{array}{r}21.0 \\
<5.0 \\
<5.0 \\
760.0 \\
3,700.0\end{array}$ \\
\hline $\begin{array}{l}80 H 3329 \mathrm{C} \\
80 \mathrm{H} 3330 \mathrm{~A} \\
80 \mathrm{H} 3330 \mathrm{~B} \\
80 \mathrm{H} 3330 \mathrm{C} \\
80 \mathrm{H} 3330 D\end{array}$ & $\begin{array}{l}N \\
N \\
N \\
N \\
N\end{array}$ & $\begin{array}{l}N \\
N \\
N \\
N \\
N\end{array}$ & $\begin{array}{l}N \\
N \\
N \\
N \\
N\end{array}$ & $\begin{array}{r}N \\
N \\
500 \\
500 \\
N\end{array}$ & $\begin{array}{r}<10 \\
15 \\
70 \\
150 \\
20\end{array}$ & $\begin{array}{l}\mathbf{N} \\
\mathbf{N} \\
\mathbf{N} \\
\mathbf{N} \\
\mathbf{N}\end{array}$ & $\begin{array}{r}70 \\
N \\
10 \\
20 \\
15\end{array}$ & $\begin{array}{r}N \\
<200 \\
500 \\
3,000 \\
2,000\end{array}$ & $\begin{array}{r}N \\
20 \\
30 \\
30 \\
30\end{array}$ & $\begin{array}{l}\mathbf{N} \\
\mathbf{N} \\
\mathbf{N} \\
\mathbf{N} \\
\mathbf{N}\end{array}$ & $\begin{array}{l}.10 \\
<.05 \\
<.05 \\
<.05 \\
<.05\end{array}$ & $\begin{array}{l}67.0 \\
27.0 \\
40.0 \\
97.0 \\
55.0\end{array}$ \\
\hline $\begin{array}{l}80 H 3330 E \\
80 H 3331 A \\
80 H 3331 B \\
80 H 3331 C \\
80 H 33310\end{array}$ & $\begin{array}{r}N \\
200 \\
100 \\
N \\
<100\end{array}$ & $\begin{array}{r}7 \\
<5 \\
5 \\
N \\
N\end{array}$ & $\begin{array}{l}N \\
N \\
N \\
N \\
N\end{array}$ & $\begin{array}{r}1,000 \\
300 \\
500 \\
100 \\
\mathrm{~N}\end{array}$ & $\begin{array}{r}200 \\
200 \\
500 \\
10 \\
100\end{array}$ & $\begin{array}{l}N \\
N \\
N \\
N \\
N\end{array}$ & $\begin{array}{r}15 \\
100 \\
500 \\
15 \\
20\end{array}$ & $\begin{array}{r}N \\
2,000 \\
N \\
N \\
200\end{array}$ & $\begin{array}{r}100 \\
N \\
15 \\
N \\
20\end{array}$ & $\begin{array}{l}N \\
N \\
N \\
N \\
N\end{array}$ & $\begin{array}{r}<.05 \\
.05 \\
.10 \\
.20 \\
.10\end{array}$ & $\begin{array}{r}10.0 \\
500.0 \\
370.0 \\
18.0 \\
87.0\end{array}$ \\
\hline $\begin{array}{l}80 H 3332 A \\
80 H 3332 B \\
80 H 3332 C \\
80 H 3332 D \\
80 H 3332 E\end{array}$ & $\begin{array}{r}N \\
N \\
N \\
3,000 \\
700\end{array}$ & $\begin{array}{l}N \\
N \\
7 \\
5 \\
N\end{array}$ & $\begin{array}{r}N \\
N \\
N \\
10,000 \\
3,000\end{array}$ & $\begin{array}{r}300 \\
N \\
<100 \\
700 \\
N\end{array}$ & $\begin{array}{r}10 \\
<10 \\
50 \\
30 \\
15\end{array}$ & $\begin{array}{l}\mathbf{N} \\
\mathbf{N} \\
\mathbf{N} \\
\mathbf{N} \\
\mathbf{N}\end{array}$ & $\begin{array}{r}N \\
N \\
30 \\
100 \\
30\end{array}$ & $\begin{array}{r}N \\
N \\
N \\
1,500 \\
200\end{array}$ & $\begin{array}{r}10 \\
N \\
100 \\
30 \\
20\end{array}$ & $\begin{array}{l}N \\
N \\
N \\
N \\
N\end{array}$ & $\begin{array}{r}.05 \\
.05 \\
<.05 \\
5.30 \\
1.20\end{array}$ & $\begin{array}{r}<5.0 \\
<5.0 \\
38.0 \\
5,400.0 \\
2,200.0\end{array}$ \\
\hline $\begin{array}{l}80 \mathrm{H} 3332 \mathrm{~F} \\
80 \mathrm{H} 3333 \\
80 \mathrm{H} 3334 \mathrm{~A} \\
80 \mathrm{H} 3334 \mathrm{~B} \\
80 \mathrm{H} 3336\end{array}$ & $\begin{array}{r}N \\
N \\
100 \\
500 \\
N\end{array}$ & $\begin{array}{l}N \\
N \\
N \\
5 \\
N\end{array}$ & $\begin{array}{r}100 \\
N \\
N \\
N \\
N\end{array}$ & $\begin{array}{r}N \\
N \\
N \\
300 \\
N\end{array}$ & $\begin{array}{r}10 \\
30 \\
20 \\
300 \\
15\end{array}$ & $\begin{array}{l}\mathbf{N} \\
\mathbf{N} \\
\mathbf{N} \\
\mathbf{N} \\
\mathbf{N}\end{array}$ & $\begin{array}{r}\mathbf{N} \\
10 \\
\mathbf{N} \\
10 \\
\mathrm{~N}\end{array}$ & $\begin{array}{r}N \\
<200 \\
<200 \\
500 \\
N\end{array}$ & $\begin{array}{r}70 \\
10 \\
30 \\
70 \\
150\end{array}$ & $\begin{array}{l}N \\
N \\
N \\
N \\
N\end{array}$ & $\begin{array}{l}.60 \\
<.05 \\
<.05 \\
<.05 \\
<.05\end{array}$ & $\begin{array}{r}21.0 \\
55.0 \\
19.0 \\
480.0 \\
7.0\end{array}$ \\
\hline
\end{tabular}


TABLE 4--RESULTS OF ANALYSES, DETROIT MINING DISTRICT, UTAH--Cont inued

\begin{tabular}{|c|c|c|c|c|c|c|c|c|c|c|}
\hline Sample & $\begin{array}{l}\text { BI -PPM } \\
\text { ICP }\end{array}$ & $\begin{array}{l}C D-P P M \\
I C P\end{array}$ & $\begin{array}{l}\text { SB-PPM } \\
I C P\end{array}$ & $\begin{array}{l}Z N-P P M \\
I C P\end{array}$ & $\begin{array}{c}\text { Na-pet. } \\
s\end{array}$ & $\begin{array}{c}\text { P-pct. } \\
\text { s }\end{array}$ & $\begin{array}{l}\text { Ga-ppm } \\
\text { s }\end{array}$ & $\begin{array}{l}\text { Ge-ppm } \\
\text { s }\end{array}$ & $\begin{array}{l}\text { HG-PPM } \\
\mathrm{CV}\end{array}$ & $\begin{array}{l}\text { F\% } \\
\text { ISE }\end{array}$ \\
\hline $\begin{array}{l}80 H 3306 \\
80 H 3307 A \\
80 H 3307 B \\
80 H 3319 A \\
80 H 3319 B\end{array}$ & $\begin{array}{r}<2.00 \\
<2.00 \\
<2.00 \\
6.00 \\
<2.00\end{array}$ & $\begin{array}{r}.200 \\
.400 \\
.400 \\
.200 \\
<.100\end{array}$ & $\begin{array}{r}3.00 \\
4.00 \\
4.00 \\
4.00 \\
<2.00\end{array}$ & $\begin{array}{r}5.00 \\
39.00 \\
<2.00 \\
4.00 \\
<2.00\end{array}$ & $\begin{array}{l}N \\
N \\
N \\
<.2 \\
<.2\end{array}$ & $\begin{array}{l}<.2 \\
<.2 \\
<.2 \\
<.2 \\
<.2\end{array}$ & $\begin{array}{r}<5 \\
10 \\
5 \\
5 \\
<5\end{array}$ & $\begin{array}{l}\mathbf{N} \\
\mathbf{N} \\
\mathbf{N} \\
\mathbf{N} \\
\mathbf{N}\end{array}$ & $\begin{array}{l}.02 \\
.68 \\
.32 \\
N \\
N\end{array}$ & $\begin{array}{l}<.01 \\
.03 \\
.01 \\
<.01 \\
<.01\end{array}$ \\
\hline $\begin{array}{l}80 H 3322 \\
8013323 \\
80 H 3325 A \\
80 H 3325 B \\
80 H 3325 C\end{array}$ & $\begin{array}{r}<2.00 \\
<2.00 \\
4.00 \\
24.00 \\
44.00\end{array}$ & $\begin{array}{l}.700 \\
.800 \\
.300 \\
2.300 \\
1.200\end{array}$ & $\begin{array}{r}<2.00 \\
<2.00 \\
13.00 \\
280.00 \\
300.00\end{array}$ & $\begin{array}{r}3.00 \\
4.00 \\
27.00 \\
41.00 \\
84.00\end{array}$ & $\begin{array}{l}N \\
N \\
<.2 \\
.5\end{array}$ & $\begin{array}{l}N \\
<.2 \\
N \\
N \\
N\end{array}$ & $\begin{array}{r}10 \\
10 \\
20 \\
10 \\
5\end{array}$ & $\begin{array}{l}N \\
N \\
N \\
N \\
N\end{array}$ & $\begin{array}{c}N \\
N \\
.10 \\
2.00 \\
13.80\end{array}$ & $\begin{array}{l}<.01 \\
.01 \\
.05 \\
<.01 \\
<.01\end{array}$ \\
\hline $\begin{array}{l}80 H 3325 D \\
80 H 3326 A \\
80 H 3326 B \\
80 H 3326 C \\
80 H 3327 A\end{array}$ & $\begin{array}{l}<2.00 \\
<2.00 \\
<2.00 \\
<2.00 \\
<2.00\end{array}$ & $\begin{array}{r}.200 \\
.600 \\
.200 \\
.200 \\
1.500\end{array}$ & $\begin{array}{r}8.00 \\
160.00 \\
15.00 \\
3.00 \\
7.00\end{array}$ & $\begin{array}{r}38.00 \\
52.00 \\
8.00 \\
<2.00 \\
6.00\end{array}$ & $\begin{array}{l}N \\
N \\
N \\
N \\
N\end{array}$ & $\begin{array}{l}<.2 \\
N \\
<.2 \\
N \\
<.2\end{array}$ & $\begin{array}{r}7 \\
10 \\
<5 \\
N \\
N\end{array}$ & $\begin{array}{l}N \\
N \\
N \\
N \\
N\end{array}$ & $\begin{array}{l}.04 \\
.40 \\
N \\
.18\end{array}$ & $\begin{array}{l}<.01 \\
<.01 \\
<.01 \\
.01 \\
<.01\end{array}$ \\
\hline $\begin{array}{l}80 H 3327 \mathrm{~B} \\
80 \mathrm{H3} 27 \mathrm{C} \\
80 \mathrm{H3} 27 \mathrm{D} \\
80 \mathrm{H} 328 \mathrm{~A} \\
80 \mathrm{H} 328 \mathrm{~B}\end{array}$ & $\begin{array}{l}<2.00 \\
<2.00 \\
<2.00 \\
<2.00 \\
<2.00\end{array}$ & $\begin{array}{l}.100 \\
.500 \\
.300 \\
4.000 \\
5.200\end{array}$ & $\begin{array}{l}<2.00 \\
<2.00 \\
<2.00 \\
<2.00 \\
<2.00\end{array}$ & $\begin{array}{l}<2.00 \\
23.00 \\
19.00 \\
50.00 \\
64.00\end{array}$ & $\begin{array}{l}N \\
.5 \\
.7 \\
.2 \\
.2\end{array}$ & $\begin{array}{c}N \\
N \\
<.2 \\
N \\
N\end{array}$ & $\begin{array}{l}N \\
30 \\
20 \\
15 \\
15\end{array}$ & $\begin{array}{l}N \\
N \\
N \\
N \\
N\end{array}$ & $\begin{array}{l}.02 \\
N \\
N \\
.12 \\
.10\end{array}$ & $\begin{array}{l}.03 \\
.07 \\
.06 \\
.03 \\
.05\end{array}$ \\
\hline $\begin{array}{l}80 H 3328 C \\
80 H 3328 D \\
8 D H 3328 E \\
80 H 3329 A \\
8 D H 3329 B\end{array}$ & $\begin{array}{r}<2.00 \\
<2.00 \\
<2.00 \\
2,500.00 \\
>20,000.00\end{array}$ & $\begin{array}{r}1.200 \\
.200 \\
6.600 \\
.500 \\
27.000\end{array}$ & $\begin{array}{r}<2.00 \\
<2.00 \\
<2.00 \\
1,800.00 \\
13,000.00\end{array}$ & $\begin{array}{r}63.00 \\
<2.00 \\
180.00 \\
19.00 \\
140.00\end{array}$ & $\begin{array}{c}N \\
N \\
.3 \\
N \\
<.2\end{array}$ & $\begin{array}{l}N \\
N \\
N \\
<.2 \\
<.2\end{array}$ & $\begin{array}{r}10 \\
N \\
15 \\
<5 \\
15\end{array}$ & $\begin{array}{l}N \\
N \\
N \\
N \\
N\end{array}$ & $\begin{array}{r}.18 \\
.10 \\
.76 \\
>36.00 \\
>36.00\end{array}$ & $\begin{array}{r}.04 \\
<.01 \\
.07 \\
<.01 \\
.02\end{array}$ \\
\hline $\begin{array}{l}80 H 3329 C \\
80 H 3330 A \\
80 H 3330 B \\
80 H 3330 C \\
8 D H 3330 D\end{array}$ & $\begin{array}{r}8.00 \\
10.00 \\
8.00 \\
9.00 \\
12.00\end{array}$ & $\begin{array}{r}1.000 \\
.600 \\
1.400 \\
8.000 \\
.800\end{array}$ & $\begin{array}{l}82.00 \\
29.00 \\
40.00 \\
59.00 \\
25.00\end{array}$ & $\begin{array}{r}37.00 \\
130.00 \\
490.00 \\
2,500.00 \\
1,200.00\end{array}$ & $\begin{array}{l}N \\
<.2 \\
.2 \\
.5 \\
.3\end{array}$ & $\begin{array}{l}N \\
<.2 \\
<.2 \\
N \\
N\end{array}$ & $\begin{array}{r}10 \\
10 \\
30 \\
70 \\
5\end{array}$ & $\begin{array}{l}N \\
N \\
N \\
N \\
N\end{array}$ & $\begin{array}{r}>36.00 \\
.90 \\
1.48 \\
2.40 \\
1.60\end{array}$ & $\begin{array}{l}.03 \\
<.01 \\
.03 \\
.08 \\
.01\end{array}$ \\
\hline $\begin{array}{l}80 H 3330 E \\
80 H 3331 A \\
80 H 3331 B \\
80 H 3331 C \\
80 H 3331 D\end{array}$ & $\begin{array}{r}7.00 \\
<2.00 \\
57.00 \\
10.00 \\
70.00\end{array}$ & $\begin{array}{r}<.100 \\
23.000 \\
1.900 \\
1.300 \\
2.000\end{array}$ & $\begin{array}{r}7.00 \\
140.00 \\
82.00 \\
7.00 \\
23.00\end{array}$ & $\begin{array}{r}37.00 \\
900.00 \\
220.00 \\
24.00 \\
190.00\end{array}$ & $\begin{array}{c}N \\
.3 \\
<.2 \\
N \\
<.2\end{array}$ & $\begin{array}{l}.5 \\
N \\
N \\
N\end{array}$ & $\begin{array}{l}50 \\
30 \\
30 \\
10 \\
15\end{array}$ & $\begin{array}{l}N \\
N \\
N \\
N \\
N\end{array}$ & $\begin{array}{r}1.04 \\
>36.00 \\
8.60 \\
.68 \\
2.40\end{array}$ & $\begin{array}{l}.10 \\
.03 \\
.03 \\
.01 \\
.01\end{array}$ \\
\hline $\begin{array}{l}80 H 3332 A \\
8 D H 3332 B \\
80 H 3332 C \\
8 D H 3332 D \\
8 D H 3332 E\end{array}$ & $\begin{array}{r}<2.00 \\
<2.00 \\
24.00 \\
>20,000.00 \\
3,800.00\end{array}$ & $\begin{array}{r}.100 \\
.100 \\
6.500 \\
21.000 \\
12.000\end{array}$ & $\begin{array}{r}3.00 \\
<2.00 \\
4.00 \\
1,900.00 \\
1,100.00\end{array}$ & $\begin{array}{r}2.00 \\
35.00 \\
26.00 \\
1.300 .00 \\
290.00\end{array}$ & $\begin{array}{l}N \\
N \\
N \\
.5 \\
<.2\end{array}$ & $\begin{array}{c}N \\
N \\
<.2 \\
N \\
N\end{array}$ & $\begin{array}{r}N \\
N \\
30 \\
20 \\
10\end{array}$ & $\begin{array}{l}N \\
N \\
N \\
N \\
N\end{array}$ & $\begin{array}{r}.22 \\
.18 \\
.46 \\
>36.00 \\
14.80\end{array}$ & $\begin{array}{l}.01 \\
.01 \\
.15 \\
.05 \\
.01\end{array}$ \\
\hline $\begin{array}{l}80 H 3332 F \\
80 H 3333 \\
80 H 3334 A \\
80 H 3334 B \\
80 H 3336\end{array}$ & $\begin{array}{r}18.00 \\
17.00 \\
8.00 \\
<2.00 \\
<2.00\end{array}$ & $\begin{array}{r}<.100 \\
.300 \\
.100 \\
1.800 \\
<.100\end{array}$ & $\begin{array}{r}17.00 \\
32.00 \\
19.00 \\
380.00 \\
5.00\end{array}$ & $\begin{array}{r}13.00 \\
110.00 \\
35.00 \\
340.00 \\
3.00\end{array}$ & $\begin{array}{l}N \\
N \\
N \\
N \\
N\end{array}$ & $\begin{array}{l}\mathrm{N} \\
\mathrm{N} \\
<.2 \\
<.2 \\
<.2\end{array}$ & $\begin{array}{r}<5 \\
7 \\
5 \\
30 \\
5\end{array}$ & $\begin{array}{l}N \\
N \\
N \\
N \\
N\end{array}$ & $\begin{array}{r}.34 \\
.68 \\
1.28 \\
.88 \\
.10\end{array}$ & $\begin{array}{l}<.01 \\
<.01 \\
<.01 \\
.05 \\
<.01\end{array}$ \\
\hline
\end{tabular}


TABLE 4--RESULTS OF ANALYSES, DETROIT MINING DISTRICT, UTAH--Continued

\begin{tabular}{|c|c|c|c|c|c|c|c|c|c|c|}
\hline Sample & Latitude & Longi tude & $\begin{array}{c}\text { Fe-pct. } \\
\text { s }\end{array}$ & $\begin{array}{c}\text { Mg-pct. } \\
\text { s }\end{array}$ & $\begin{array}{c}\text { Ca-pct. } \\
s\end{array}$ & $\begin{array}{c}\text { Ti-pct. } \\
\text { s }\end{array}$ & $\begin{array}{c}\text { Mn-ppm } \\
\mathrm{s}\end{array}$ & $\begin{array}{c}A g-p p m \\
s\end{array}$ & $\begin{array}{l}\text { As - ppm } \\
s\end{array}$ & $\begin{array}{c}A u-p p m \\
s\end{array}$ \\
\hline $\begin{array}{l}80 H 3337 A \\
80 H 3337 B \\
80 H 3339 A \\
80 H 3339 B \\
80 H 3340\end{array}$ & $\begin{array}{lll}39 & 31 & 40 \\
39 & 31 & 40 \\
39 & 31 & 38 \\
39 & 31 & 38 \\
39 & 31 & 35\end{array}$ & $\begin{array}{rrr}112 & 59 & 54 \\
112 & 59 & 54 \\
113 & 0 & 11 \\
113 & 0 & 11 \\
113 & 0 & 4\end{array}$ & $\begin{array}{r}7.00 \\
5.00 \\
5.00 \\
2.00 \\
.20\end{array}$ & $\begin{array}{r}.07 \\
.05 \\
.05 \\
10.00 \\
.02\end{array}$ & $\begin{array}{r}.07 \\
.10 \\
.20 \\
>20.00 \\
.05\end{array}$ & $\begin{array}{l}.100 \\
.100 \\
.150 \\
.005 \\
.150\end{array}$ & $\begin{array}{r}50 \\
70 \\
50 \\
3,000 \\
15\end{array}$ & $\begin{array}{l}\mathrm{N} \\
\mathrm{N} \\
.05 \\
\mathrm{~N}\end{array}$ & $\begin{array}{l}N \\
N \\
N \\
N \\
N\end{array}$ & $\begin{array}{l}\mathbf{N} \\
\mathbf{N} \\
\mathbf{N} \\
\mathbf{N} \\
\mathbf{N}\end{array}$ \\
\hline $\begin{array}{l}80 H 3342 \\
80 H 3343 \\
80 H 3344 \\
80 H 3345 \\
80 H 3346 A\end{array}$ & $\begin{array}{rrr}39 & 33 & 6 \\
39 & 33 & 14 \\
39 & 33 & 6 \\
39 & 33 & 10 \\
39 & 31 & 33\end{array}$ & $\begin{array}{ccc}113 & 1 & 57 \\
113 & 1 & 53 \\
113 & 2 & 3 \\
113 & 2 & 4 \\
113 & 0 & 0\end{array}$ & $\begin{array}{l}.05 \\
<.05 \\
1.50 \\
1.00 \\
3.00\end{array}$ & $\begin{array}{r}7.00 \\
5.00 \\
10.00 \\
3.00 \\
<.02\end{array}$ & $\begin{array}{r}>20.00 \\
>20.00 \\
>20.00 \\
>20.00 \\
.10\end{array}$ & $\begin{array}{r}<.002 \\
<.002 \\
.003 \\
.100 \\
<.002\end{array}$ & $\begin{array}{r}70 \\
70 \\
1,500 \\
100 \\
300\end{array}$ & $\begin{array}{l}\mathrm{N} \\
\mathrm{N} \\
\mathrm{N} \\
\mathrm{N} \\
\mathrm{N}\end{array}$ & $\begin{array}{l}N \\
N \\
N \\
N \\
N\end{array}$ & $\begin{array}{l}N \\
N \\
N \\
N \\
N\end{array}$ \\
\hline $\begin{array}{l}80 H 33468 \\
80 H 3346 C \\
80 H 33460 \\
8013347 \\
80 H 3348 A\end{array}$ & $\begin{array}{lll}39 & 31 & 33 \\
39 & 31 & 33 \\
39 & 31 & 33 \\
39 & 31 & 31 \\
39 & 31 & 33\end{array}$ & $\begin{array}{rrr}113 & 0 & 0 \\
113 & 0 & 0 \\
113 & 0 & 0 \\
112 & 59 & 58 \\
112 & 59 & 54\end{array}$ & $\begin{array}{r}1.00 \\
20.00 \\
3.00 \\
7.00 \\
2.00\end{array}$ & $\begin{array}{r}.02 \\
.07 \\
>10.00 \\
.15 \\
1.00\end{array}$ & $\begin{array}{r}.07 \\
.07 \\
>20.00 \\
.70 \\
.20\end{array}$ & $\begin{array}{r}.200 \\
.150 \\
<.002 \\
.020 \\
.300\end{array}$ & $\begin{array}{r}300 \\
50 \\
3,000 \\
100 \\
300\end{array}$ & $\begin{array}{l}N \\
<.50 \\
N \\
<.50 \\
N\end{array}$ & $\begin{array}{r}N \\
<200 \\
N \\
300 \\
N\end{array}$ & $\begin{array}{l}N \\
N \\
N \\
N \\
N\end{array}$ \\
\hline $\begin{array}{l}80 H 3348 B \\
80 H 3349 \\
80 H 3350 A \\
80 H 3350 B \\
80 H 3350 C\end{array}$ & $\begin{array}{lll}39 & 31 & 33 \\
39 & 31 & 30 \\
39 & 31 & 27 \\
39 & 31 & 27 \\
39 & 31 & 27\end{array}$ & $\begin{array}{lll}112 & 59 & 54 \\
112 & 59 & 54 \\
112 & 59 & 52 \\
112 & 59 & 52 \\
112 & 59 & 52\end{array}$ & $\begin{array}{r}3.00 \\
.70 \\
1.00 \\
1.50 \\
7.00\end{array}$ & $\begin{array}{l}.70 \\
.30 \\
.50 \\
.50 \\
.70\end{array}$ & $\begin{array}{r}.30 \\
20.00 \\
20.00 \\
.15 \\
.70\end{array}$ & $\begin{array}{l}.150 \\
.005 \\
.100 \\
.070 \\
.020\end{array}$ & $\begin{array}{r}1,500 \\
1,000 \\
1,500 \\
1,000 \\
>5,000\end{array}$ & $\begin{array}{l}\mathrm{N} \\
\mathrm{N} \\
\mathrm{N} \\
\mathrm{N} \\
\mathrm{N}\end{array}$ & $\begin{array}{l}N \\
N \\
N \\
N \\
N\end{array}$ & $\begin{array}{l}N \\
N \\
N \\
N \\
N\end{array}$ \\
\hline $\begin{array}{l}80 H 3351 \\
80 H 3353 \\
80 H 3354 \\
80 H 3355 \\
8013363\end{array}$ & $\begin{array}{lll}39 & 31 & 27 \\
39 & 31 & 39 \\
39 & 31 & 39 \\
39 & 31 & 41 \\
39 & 34 & 21\end{array}$ & $\begin{array}{rrr}112 & 59 & 55 \\
113 & 0 & 22 \\
113 & 0 & 22 \\
113 & 0 & 19 \\
112 & 58 & 32\end{array}$ & $\begin{array}{r}5.00 \\
.15 \\
1.00 \\
5.00 \\
5.00\end{array}$ & $\begin{array}{l}.05 \\
<.02 \\
1.50 \\
1.00 \\
7.00\end{array}$ & $\begin{array}{l}.20 \\
<.05 \\
.05 \\
1.00 \\
2.00\end{array}$ & $\begin{array}{r}<.002 \\
.300 \\
1.000 \\
.200 \\
.300\end{array}$ & $\begin{array}{r}500 \\
200 \\
70 \\
700 \\
700\end{array}$ & $\begin{array}{l}2.00 \\
N \\
N \\
N \\
.50\end{array}$ & $\begin{array}{l}N \\
N \\
N \\
N \\
N\end{array}$ & $\begin{array}{l}N \\
N \\
N \\
N \\
N\end{array}$ \\
\hline $\begin{array}{l}80 H 3369 \\
80 H 3377 \\
80 H 3378 \\
80 H 3379 \\
80 H 3380\end{array}$ & $\begin{array}{lll}39 & 34 & 16 \\
39 & 33 & 55 \\
39 & 33 & 42 \\
39 & 33 & 43 \\
39 & 33 & 36\end{array}$ & $\begin{array}{rrr}113 & 0 & 4 \\
113 & 0 & 50 \\
113 & 0 & 53 \\
113 & 0 & 46 \\
113 & 1 & 2\end{array}$ & $\begin{array}{r}.70 \\
.70 \\
20.00 \\
5.00 \\
5.00\end{array}$ & $\begin{array}{l}<.02 \\
<.02 \\
.03 \\
.15 \\
.02\end{array}$ & $\begin{array}{r}.30 \\
<.05 \\
.20 \\
.05 \\
.30\end{array}$ & $\begin{array}{l}.050 \\
.020 \\
.020 \\
.500 \\
.100\end{array}$ & $\begin{array}{r}15 \\
<10 \\
<10 \\
10 \\
70\end{array}$ & $\begin{array}{l}\mathrm{N} \\
\mathrm{N} \\
\mathrm{N} \\
\mathrm{N} \\
\mathrm{N}\end{array}$ & $\begin{array}{r}N \\
N \\
200 \\
N \\
N\end{array}$ & $\begin{array}{l}N \\
N \\
N \\
N \\
N\end{array}$ \\
\hline $\begin{array}{l}80 H 3381 \\
80 H 3385 \\
80 H 3386 \\
80 H 3388 \\
80 I 3390 A\end{array}$ & $\begin{array}{lll}39 & 33 & 38 \\
39 & 33 & 28 \\
39 & 33 & 18 \\
39 & 33 & 19 \\
39 & 33 & 20\end{array}$ & $\begin{array}{rrr}113 & 1 & 12 \\
113 & 0 & 42 \\
113 & 0 & 40 \\
113 & 0 & 10 \\
112 & 59 & 51\end{array}$ & $\begin{array}{r}20.00 \\
3.00 \\
7.00 \\
3.00 \\
.05\end{array}$ & $\begin{array}{l}.02 \\
.03 \\
<.02 \\
<.02 \\
<.02\end{array}$ & $\begin{array}{r}.30 \\
.50 \\
.07 \\
<.05 \\
.20\end{array}$ & $\begin{array}{l}.150 \\
.300 \\
.100 \\
.050 \\
.500\end{array}$ & $\begin{array}{r}<10 \\
10 \\
15 \\
20 \\
100\end{array}$ & $\begin{array}{l}\mathrm{N} \\
\mathrm{N} \\
\mathrm{N} \\
\mathrm{N} \\
\mathrm{N}\end{array}$ & $\begin{array}{l}N \\
N \\
N \\
N \\
N\end{array}$ & $\begin{array}{l}\mathbf{N} \\
\mathbf{N} \\
\mathbf{N} \\
\mathbf{N} \\
\mathbf{N}\end{array}$ \\
\hline $\begin{array}{l}8013390 B \\
8013390 C \\
80 H 3394 \\
80 H 3398 \\
80 H 3406\end{array}$ & $\begin{array}{rrr}39 & 33 & 20 \\
39 & 33 & 20 \\
39 & 33 & 9 \\
39 & 32 & 54 \\
39 & 32 & 51\end{array}$ & $\begin{array}{lll}112 & 59 & 51 \\
112 & 59 & 51 \\
113 & 0 & 40 \\
113 & 0 & 20 \\
113 & 0 & 15\end{array}$ & $\begin{array}{r}3.00 \\
.10 \\
7.00 \\
15.00 \\
.20\end{array}$ & $\begin{array}{l}<.02 \\
<.02 \\
<.02 \\
.03 \\
<.02\end{array}$ & $\begin{array}{r}.10 \\
<.05 \\
<.05 \\
.15 \\
.07\end{array}$ & $\begin{array}{l}.300 \\
.100 \\
.200 \\
.150 \\
.500\end{array}$ & $\begin{array}{r}50 \\
50 \\
N \\
70 \\
1,000\end{array}$ & $\begin{array}{l}<.50 \\
N \\
<.50 \\
N \\
N\end{array}$ & $\begin{array}{l}\mathrm{N} \\
\mathrm{N} \\
\mathrm{N} \\
\mathrm{N} \\
\mathrm{N}\end{array}$ & $\begin{array}{l}N \\
N \\
N \\
N \\
N\end{array}$ \\
\hline $\begin{array}{l}80 H 3408 \\
80 H 3409 \\
8013410 \\
8013411 \\
80 H 3413\end{array}$ & $\begin{array}{rrr}39 & 33 & 1 \\
39 & 33 & 2 \\
39 & 32 & 39 \\
39 & 32 & 41 \\
39 & 32 & 45\end{array}$ & $\begin{array}{rrr}113 & 0 & 10 \\
113 & 0 & 5 \\
112 & 59 & 42 \\
112 & 59 & 36 \\
113 & 0 & 3\end{array}$ & $\begin{array}{r}5.00 \\
.20 \\
.50 \\
10.00 \\
.15\end{array}$ & $\begin{array}{l}.02 \\
<.02 \\
.15 \\
<.02 \\
<.02\end{array}$ & $\begin{array}{r}.10 \\
.07 \\
.05 \\
<.05 \\
.07\end{array}$ & $\begin{array}{l}.500 \\
.200 \\
.300 \\
.500 \\
.700\end{array}$ & $\begin{array}{r}200 \\
20 \\
20 \\
50 \\
30\end{array}$ & $\begin{array}{l}N \\
N \\
<.50 \\
<.50 \\
N\end{array}$ & $\begin{array}{r}N \\
N \\
N \\
700 \\
N\end{array}$ & $\begin{array}{l}N \\
N \\
N \\
N \\
N\end{array}$ \\
\hline
\end{tabular}


TABLE 4--RESULTS OF ANALYSES, DETROIT MINING DISTRICT, UTAH--Continued

\begin{tabular}{|c|c|c|c|c|c|c|c|c|c|c|c|c|c|}
\hline Sample & $\begin{array}{c}\text { B-ppm } \\
S\end{array}$ & $\begin{array}{c}\text { Ba-ppm } \\
s\end{array}$ & $\begin{array}{c}\text { Be-ppm } \\
s\end{array}$ & $\begin{array}{c}B i-p p m \\
s\end{array}$ & $\begin{array}{c}\text { Cd-ppm } \\
\mathrm{s}\end{array}$ & $\begin{array}{c}\text { Co-ppm } \\
s\end{array}$ & $\begin{array}{c}\mathrm{Cr}-\mathrm{ppm} \\
\mathrm{s}\end{array}$ & $\begin{array}{c}\text { Cu-ppm } \\
s\end{array}$ & $\begin{array}{l}\text { La-ppm } \\
\mathrm{s}\end{array}$ & $\begin{array}{c}\text { Mo-ppm } \\
\mathrm{s}\end{array}$ & $\begin{array}{c}\text { Nb-ppm } \\
\text { s }\end{array}$ & $\begin{array}{c}\text { Ni-ppm } \\
s\end{array}$ & $\begin{array}{c}\text { Pb-ppm } \\
\text { s }\end{array}$ \\
\hline $\begin{array}{l}80 H 3337 A \\
80 H 3337 B \\
80 H 3339 A \\
80 H 3339 B \\
80 H 3340\end{array}$ & $\begin{array}{r}20 \\
20 \\
15 \\
N \\
10\end{array}$ & $\begin{array}{r}100 \\
2,000 \\
300 \\
20 \\
300\end{array}$ & $\begin{array}{r}1.5 \\
<1.0 \\
N \\
N \\
N\end{array}$ & $\begin{array}{l}N \\
N \\
N \\
N \\
N\end{array}$ & $\begin{array}{l}N \\
N \\
N \\
N \\
N\end{array}$ & $\begin{array}{l}N \\
N \\
N \\
N \\
N\end{array}$ & $\begin{array}{r}10 \\
15 \\
30 \\
N \\
<10\end{array}$ & $\begin{array}{r}5 \\
5 \\
20 \\
<5 \\
7\end{array}$ & $\begin{array}{r}N \\
<50 \\
<50 \\
N \\
N\end{array}$ & $\begin{array}{l}N \\
N \\
7 \\
N \\
N\end{array}$ & $\begin{array}{l}N \\
N \\
N \\
N \\
N\end{array}$ & $\begin{array}{l}<5 \\
<5 \\
<5 \\
N \\
5\end{array}$ & $\begin{array}{r}N \\
<10 \\
20 \\
<10 \\
10\end{array}$ \\
\hline $\begin{array}{l}80 H 3342 \\
80 H 3343 \\
80 H 3344 \\
80 H 3345 \\
80 H 3346 A\end{array}$ & $\begin{array}{r}<10 \\
<10 \\
<10 \\
30 \\
10\end{array}$ & $\begin{array}{r}N \\
N \\
70 \\
100 \\
70\end{array}$ & $\begin{array}{l}N \\
N \\
N \\
N \\
2.0\end{array}$ & $\begin{array}{l}N \\
N \\
N \\
N \\
N\end{array}$ & $\begin{array}{l}N \\
N \\
N \\
N \\
N\end{array}$ & $\begin{array}{c}N \\
N \\
N \\
N \\
10\end{array}$ & $\begin{array}{r}N \\
N \\
N \\
20 \\
N\end{array}$ & $\begin{array}{r}<5 \\
7 \\
5 \\
20 \\
20\end{array}$ & $\begin{array}{l}N \\
N \\
N \\
N \\
N\end{array}$ & $\begin{array}{l}N \\
N \\
N \\
N \\
N\end{array}$ & $\begin{array}{l}N \\
N \\
N \\
N \\
N\end{array}$ & $\begin{array}{r}N \\
N \\
<5 \\
10 \\
50\end{array}$ & $\begin{array}{r}<10 \\
10 \\
<10 \\
<10 \\
150\end{array}$ \\
\hline $\begin{array}{l}80 H 3346 B \\
80 H 3346 C \\
80 H 3346 D \\
8013347 \\
80 H 3348 A\end{array}$ & $\begin{array}{r}15 \\
<10 \\
N \\
10 \\
15\end{array}$ & $\begin{array}{r}50 \\
70 \\
70 \\
100 \\
1,000\end{array}$ & $\begin{array}{c}<1.0 \\
N \\
N \\
<1.0 \\
<1.0\end{array}$ & $\begin{array}{l}N \\
N \\
N \\
N \\
N\end{array}$ & $\begin{array}{l}N \\
N \\
N \\
N \\
N\end{array}$ & $\begin{array}{r}N \\
N \\
N \\
<10 \\
10\end{array}$ & $\begin{array}{r}15 \\
10 \\
N \\
<10 \\
<10\end{array}$ & $\begin{array}{r}7 \\
20 \\
10 \\
20 \\
7\end{array}$ & $\begin{array}{r}100 \\
200 \\
N \\
<50 \\
50\end{array}$ & $\begin{array}{r}N \\
N \\
N \\
<5 \\
N\end{array}$ & $\begin{array}{l}N \\
N \\
N \\
N \\
N\end{array}$ & $\begin{array}{r}5 \\
5 \\
<5 \\
15 \\
5\end{array}$ & $\begin{array}{r}100 \\
200 \\
150 \\
200 \\
70\end{array}$ \\
\hline $\begin{array}{l}80 H 33488 \\
80 H 3349 \\
80 H 3350 A \\
80 H 3350 B \\
80 H 3350 C\end{array}$ & $\begin{array}{r}30 \\
N \\
10 \\
50 \\
15\end{array}$ & $\begin{array}{r}500 \\
100 \\
300 \\
200 \\
1,000\end{array}$ & $\begin{array}{r}5.0 \\
N \\
<1.0 \\
3.0 \\
<1.0\end{array}$ & $\begin{array}{l}N \\
N \\
N \\
N \\
N\end{array}$ & $\begin{array}{l}N \\
N \\
N \\
N \\
N\end{array}$ & $\begin{array}{r}20 \\
N \\
N \\
50 \\
100\end{array}$ & $\begin{array}{r}20 \\
N \\
10 \\
20 \\
<10\end{array}$ & $\begin{array}{r}500 \\
5 \\
7 \\
20 \\
10\end{array}$ & $\begin{array}{r}<50 \\
N \\
N \\
N \\
70\end{array}$ & $\begin{array}{r}<5 \\
N \\
N \\
N \\
30\end{array}$ & $\begin{array}{l}N \\
N \\
N \\
N \\
N\end{array}$ & $\begin{array}{r}15 \\
<5 \\
5 \\
150 \\
70\end{array}$ & $\begin{array}{r}50 \\
200 \\
50 \\
500 \\
150\end{array}$ \\
\hline $\begin{array}{l}80 H 3351 \\
80 H 3353 \\
80 H 3354 \\
80 H 3355 \\
8013363\end{array}$ & $\begin{array}{r}10 \\
10 \\
50 \\
30 \\
<10\end{array}$ & $\begin{array}{r}200 \\
70 \\
1,500 \\
700 \\
1,000\end{array}$ & $\begin{array}{r}5.0 \\
1.5 \\
1.0 \\
1.0 \\
<1.0\end{array}$ & $\begin{array}{l}N \\
N \\
N \\
N \\
N\end{array}$ & $\begin{array}{l}N \\
N \\
N \\
N \\
N\end{array}$ & $\begin{array}{r}<10 \\
N \\
N \\
<10 \\
30\end{array}$ & $\begin{array}{r}15 \\
15 \\
50 \\
<10 \\
50\end{array}$ & $\begin{array}{r}15 \\
<5 \\
5 \\
<5 \\
150\end{array}$ & $\begin{array}{r}N \\
N \\
70 \\
N \\
N\end{array}$ & $\begin{array}{r}<5 \\
N \\
N \\
N \\
50\end{array}$ & $\begin{array}{l}N \\
N \\
N \\
N \\
N\end{array}$ & $\begin{array}{r}50 \\
5 \\
7 \\
10 \\
30\end{array}$ & $\begin{array}{r}200 \\
N \\
30 \\
<10 \\
20\end{array}$ \\
\hline $\begin{array}{l}80 H 3369 \\
80 H 3377 \\
80 H 3378 \\
80 H 3379 \\
80 H 3380\end{array}$ & $\begin{array}{r}10 \\
10 \\
N \\
15 \\
N\end{array}$ & $\begin{array}{r}100 \\
70 \\
70 \\
700 \\
300\end{array}$ & $\begin{array}{l}N \\
N \\
N \\
N \\
N\end{array}$ & $\begin{array}{l}N \\
N \\
N \\
N \\
N\end{array}$ & $\begin{array}{l}N \\
N \\
N \\
N \\
N\end{array}$ & $\begin{array}{l}N \\
N \\
N \\
N \\
N\end{array}$ & $\begin{array}{r}N \\
N \\
20 \\
<10 \\
N\end{array}$ & $\begin{array}{r}<5 \\
5 \\
1,000 \\
30 \\
30\end{array}$ & $\begin{array}{r}<50 \\
N \\
N \\
<50 \\
N\end{array}$ & $\begin{array}{l}N \\
N \\
N \\
N \\
N\end{array}$ & $\begin{array}{l}N \\
N \\
N \\
N \\
N\end{array}$ & $\begin{array}{l}<5 \\
<5 \\
<5 \\
<5 \\
<5\end{array}$ & $\begin{array}{r}<10 \\
<10 \\
10 \\
200 \\
50\end{array}$ \\
\hline $\begin{array}{l}80 H 3381 \\
80 H 3385 \\
80 H 3386 \\
80 H 3388 \\
8 D 13390 A\end{array}$ & $\begin{aligned} & N \\
< & 10 \\
< & 10 \\
< & 10 \\
< & 10\end{aligned}$ & $\begin{array}{l}500 \\
200 \\
200 \\
150 \\
200\end{array}$ & $\begin{array}{l}N \\
N \\
N \\
N \\
N\end{array}$ & $\begin{array}{l}N \\
N \\
N \\
N \\
N\end{array}$ & $\begin{array}{l}N \\
N \\
N \\
N \\
N\end{array}$ & $\begin{array}{l}N \\
N \\
N \\
N \\
N\end{array}$ & $\begin{array}{r}10 \\
10 \\
10 \\
<10 \\
N\end{array}$ & $\begin{array}{r}30 \\
15 \\
200 \\
5 \\
<5\end{array}$ & $\begin{array}{l}N \\
N \\
N \\
N \\
N\end{array}$ & $\begin{array}{l}N \\
N \\
N \\
N \\
N\end{array}$ & $\begin{array}{l}N \\
N \\
N \\
N \\
N\end{array}$ & $\begin{array}{l}N \\
<5 \\
<5 \\
<5 \\
<5\end{array}$ & $\begin{array}{l}100 \\
150 \\
500 \\
<10 \\
<10\end{array}$ \\
\hline $\begin{array}{l}80133908 \\
8013390 \mathrm{C} \\
80 \mathrm{H} 394 \\
80 \mathrm{H3} 988 \\
80 \mathrm{H} 306\end{array}$ & $\begin{array}{r}<10 \\
<10 \\
N \\
N \\
<10\end{array}$ & $\begin{array}{r}2,000 \\
200 \\
500 \\
300 \\
100\end{array}$ & $\begin{array}{l}N \\
N \\
N \\
N \\
N\end{array}$ & $\begin{array}{l}N \\
N \\
N \\
N \\
N\end{array}$ & $\begin{array}{l}N \\
N \\
N \\
N \\
N\end{array}$ & $\begin{array}{l}N \\
N \\
N \\
N \\
N\end{array}$ & $\begin{array}{r}30 \\
N \\
50 \\
10 \\
20\end{array}$ & $\begin{array}{r}7 \\
N \\
30 \\
20 \\
7\end{array}$ & $\begin{array}{r}50 \\
N \\
70 \\
N \\
N\end{array}$ & $\begin{array}{l}7 \\
N \\
N \\
5 \\
N\end{array}$ & $\begin{array}{r}<20 \\
N \\
N \\
N \\
N\end{array}$ & $\begin{array}{l}<5 \\
<5 \\
N \\
N \\
<5\end{array}$ & $\begin{array}{r}30 \\
<10 \\
2,000 \\
100 \\
<10\end{array}$ \\
\hline $\begin{array}{l}80 H 3408 \\
80 H 3409 \\
8013410 \\
8013411 \\
80 H 3413\end{array}$ & $\begin{array}{r}<10 \\
10 \\
20 \\
N \\
10\end{array}$ & $\begin{array}{r}500 \\
200 \\
300 \\
50 \\
50\end{array}$ & $\begin{array}{c}N \\
<1.0 \\
<1.0 \\
N \\
N\end{array}$ & $\begin{array}{r}N \\
N \\
N \\
200 \\
N\end{array}$ & $\begin{array}{l}N \\
N \\
N \\
N \\
N\end{array}$ & $\begin{array}{r}N \\
N \\
N \\
<10 \\
N\end{array}$ & $\begin{array}{r}30 \\
<10 \\
50 \\
100 \\
20\end{array}$ & $\begin{array}{r}5 \\
<5 \\
7,000 \\
700 \\
30\end{array}$ & $\begin{array}{r}<50 \\
<50 \\
<50 \\
N \\
N\end{array}$ & $\begin{array}{l}7 \\
N \\
N \\
5 \\
N\end{array}$ & $\begin{array}{r}N \\
N \\
N \\
<20 \\
<20\end{array}$ & $\begin{array}{r}N \\
<5 \\
<5 \\
20 \\
N\end{array}$ & $\begin{array}{r}15 \\
<10 \\
20 \\
150 \\
N\end{array}$ \\
\hline
\end{tabular}


TABLE 4--RESULTS OF ANALYSES, DETROIT MINING DISTRICT, UTAH--Cont inUed

\begin{tabular}{|c|c|c|c|c|c|c|c|c|c|c|c|c|}
\hline Sample & $\begin{array}{c}\text { sb-ppm } \\
\text { s }\end{array}$ & $\begin{array}{c}\text { Sc-ppm } \\
\text { s }\end{array}$ & $\begin{array}{c}\text { sn-ppm } \\
s\end{array}$ & $\begin{array}{c}\text { sr-ppm } \\
s\end{array}$ & $\begin{array}{c}v-p p m \\
s\end{array}$ & $\begin{array}{c}W-p p m \\
s\end{array}$ & $\underset{S}{Y-p p m}$ & $\begin{array}{c}2 n-p p m \\
s\end{array}$ & $\begin{array}{c}2 r-p p m \\
s\end{array}$ & $\begin{array}{c}\text { Th-ppm } \\
s\end{array}$ & $\begin{array}{c}\text { Au-ppm } \\
\text { aa }\end{array}$ & $\begin{array}{l}\text { AS-PPM } \\
\text { ICP }\end{array}$ \\
\hline $\begin{array}{l}80 H 3337 A \\
80 H 3337 B \\
80 H 3339 A \\
80 H 3339 B \\
80 H 3340\end{array}$ & $\begin{array}{l}\mathbf{N} \\
\mathbf{N} \\
\mathbf{N} \\
\mathbf{N} \\
\mathbf{N}\end{array}$ & $\begin{array}{r}7 \\
<5 \\
<5 \\
N \\
N\end{array}$ & $\begin{array}{l}N \\
N \\
N \\
N \\
N\end{array}$ & $\begin{array}{r}N \\
150 \\
500 \\
<100 \\
200\end{array}$ & $\begin{array}{r}50 \\
20 \\
30 \\
<10 \\
15\end{array}$ & $\begin{array}{l}N \\
N \\
N \\
N \\
N\end{array}$ & $\begin{array}{l}30 \\
50 \\
30 \\
N \\
N\end{array}$ & $\begin{array}{l}N \\
N \\
N \\
N \\
N\end{array}$ & $\begin{array}{r}200 \\
200 \\
500 \\
20 \\
150\end{array}$ & $\begin{array}{l}N \\
N \\
N \\
N \\
N\end{array}$ & $\begin{array}{l}<.05 \\
<.05 \\
.30 \\
<.05 \\
.20\end{array}$ & $\begin{array}{r}5.0 \\
7.0 \\
27.0 \\
<5.0 \\
10.0\end{array}$ \\
\hline $\begin{array}{l}80 H 3342 \\
80 H 3343 \\
80 H 3344 \\
80 H 3345 \\
80 H 3346 A\end{array}$ & $\begin{array}{l}N \\
N \\
N \\
N \\
N\end{array}$ & $\begin{array}{r}N \\
N \\
N \\
<5 \\
N\end{array}$ & $\begin{array}{l}N \\
N \\
N \\
N \\
N\end{array}$ & $\begin{array}{r}150 \\
200 \\
100 \\
500 \\
N\end{array}$ & $\begin{array}{r}<10 \\
10 \\
15 \\
20 \\
70\end{array}$ & $\begin{array}{l}N \\
N \\
N \\
N \\
N\end{array}$ & $\begin{array}{r}N \\
N \\
N \\
10 \\
N\end{array}$ & $\begin{array}{l}N \\
N \\
N \\
N \\
N\end{array}$ & $\begin{array}{r}N \\
N \\
N \\
20 \\
<10\end{array}$ & $\begin{array}{l}N \\
N \\
N \\
N \\
N\end{array}$ & $\begin{array}{l}<.05 \\
<.05 \\
<.05 \\
<.05 \\
<.05\end{array}$ & $\begin{array}{l}<5.0 \\
20.0 \\
44.0 \\
13.0 \\
33.0\end{array}$ \\
\hline $\begin{array}{l}80 H 3346 B \\
80 H 3346 C \\
80 H 33460 \\
8013347 \\
80 H 3348 A\end{array}$ & $\begin{array}{r}N \\
<100 \\
N \\
N \\
N\end{array}$ & $\begin{array}{r}<5 \\
5 \\
N \\
N \\
5\end{array}$ & $\begin{array}{l}N \\
N \\
N \\
N \\
N\end{array}$ & $\begin{array}{l}N \\
N \\
N \\
N \\
N\end{array}$ & $\begin{array}{r}100 \\
200 \\
15 \\
100 \\
70\end{array}$ & $\begin{array}{l}N \\
N \\
N \\
N \\
N\end{array}$ & $\begin{array}{r}70 \\
50 \\
N \\
50 \\
15\end{array}$ & $\begin{array}{r}<200 \\
200 \\
N \\
N \\
N\end{array}$ & $\begin{array}{r}100 \\
100 \\
N \\
100 \\
100\end{array}$ & $\begin{array}{l}N \\
N \\
N \\
N \\
N\end{array}$ & $\begin{array}{r}<.05 \\
.10 \\
<.05 \\
.20 \\
<.05\end{array}$ & $\begin{array}{r}32.0 \\
230.0 \\
45.0 \\
470.0 \\
46.0\end{array}$ \\
\hline $\begin{array}{l}80 H 3348 B \\
80 H 3349 \\
80 H 3350 A \\
80 H 3350 B \\
80 H 3350 C\end{array}$ & $\begin{array}{l}N \\
N \\
N \\
N \\
N\end{array}$ & $\begin{array}{r}<5 \\
N \\
N \\
<5 \\
N\end{array}$ & $\begin{array}{l}N \\
N \\
N \\
N \\
N\end{array}$ & $\begin{array}{r}N \\
N \\
N \\
1,500 \\
500\end{array}$ & $\begin{array}{r}50 \\
50 \\
15 \\
150 \\
100\end{array}$ & $\begin{array}{l}N \\
N \\
N \\
N \\
N\end{array}$ & $\begin{array}{r}30 \\
N \\
15 \\
100 \\
20\end{array}$ & $\begin{array}{l}N \\
N \\
N \\
N \\
N\end{array}$ & $\begin{array}{r}150 \\
<10 \\
150 \\
70 \\
100\end{array}$ & $\begin{array}{l}N \\
N \\
N \\
N \\
N\end{array}$ & $\begin{array}{r}<.05 \\
.10 \\
.10 \\
.10 \\
.10\end{array}$ & $\begin{array}{r}92.0 \\
84.0 \\
86.0 \\
230.0 \\
430.0\end{array}$ \\
\hline $\begin{array}{l}80 H 3351 \\
80 H 3353 \\
80 H 3354 \\
80 H 3355 \\
8013363\end{array}$ & $\begin{array}{l}N \\
N \\
N \\
N \\
N\end{array}$ & $\begin{array}{c}N \\
N \\
10 \\
<5 \\
10\end{array}$ & $\begin{array}{l}N \\
N \\
N \\
N \\
N\end{array}$ & $\begin{array}{r}N \\
N \\
200 \\
N \\
N\end{array}$ & $\begin{array}{r}150 \\
15 \\
100 \\
20 \\
30\end{array}$ & $\begin{array}{l}N \\
N \\
N \\
N \\
N\end{array}$ & $\begin{array}{r}N \\
<10 \\
20 \\
50 \\
15\end{array}$ & $\begin{array}{r}N \\
N \\
N \\
N \\
500\end{array}$ & $\begin{array}{r}N \\
70 \\
100 \\
300 \\
100\end{array}$ & $\begin{array}{l}N \\
N \\
N \\
N \\
N\end{array}$ & $\begin{array}{r}.05 \\
.10 \\
<.05 \\
<.05 \\
<.05\end{array}$ & $\begin{array}{r}120.0 \\
19.0 \\
<5.0 \\
<5.0 \\
<5.0\end{array}$ \\
\hline $\begin{array}{l}80 H 3369 \\
80 H 3377 \\
80 H 3378 \\
80 H 3379 \\
80 H 3380\end{array}$ & $\begin{array}{l}N \\
N \\
N \\
N \\
N\end{array}$ & $\begin{array}{l}N \\
N \\
N \\
7 \\
N\end{array}$ & $\begin{array}{l}N \\
N \\
N \\
N \\
N\end{array}$ & $\begin{array}{r}100 \\
N \\
N \\
100 \\
<100\end{array}$ & $\begin{array}{r}10 \\
15 \\
50 \\
200 \\
20\end{array}$ & $\begin{array}{l}N \\
N \\
N \\
N \\
N\end{array}$ & $\begin{array}{r}N \\
N \\
N \\
10 \\
N\end{array}$ & $\begin{array}{l}N \\
N \\
N \\
N \\
N\end{array}$ & $\begin{array}{r}50 \\
30 \\
20 \\
200 \\
30\end{array}$ & $\begin{array}{l}N \\
N \\
N \\
N \\
N\end{array}$ & $\begin{array}{l}N \\
N \\
N \\
N \\
N\end{array}$ & $\begin{array}{r}9.0 \\
<5.0 \\
450.0 \\
51.0 \\
64.0\end{array}$ \\
\hline $\begin{array}{l}80 H 3381 \\
80 H 3385 \\
80 H 3386 \\
80 H 3388 \\
8013390 A\end{array}$ & $\begin{array}{l}N \\
N \\
N \\
N \\
N\end{array}$ & $\begin{array}{r}N \\
<5 \\
N \\
N \\
<5\end{array}$ & $\begin{array}{l}N \\
N \\
N \\
N \\
N\end{array}$ & $\begin{array}{r}<100 \\
N \\
200 \\
N \\
N\end{array}$ & $\begin{array}{r}70 \\
150 \\
20 \\
15 \\
10\end{array}$ & $\begin{array}{l}N \\
N \\
N \\
N \\
N\end{array}$ & $\begin{array}{r}N \\
<10 \\
N \\
N \\
10\end{array}$ & $\begin{array}{l}N \\
N \\
N \\
N \\
N\end{array}$ & $\begin{array}{r}200 \\
30 \\
30 \\
100 \\
200\end{array}$ & $\begin{array}{l}N \\
N \\
N \\
N \\
N\end{array}$ & $\begin{array}{l}N \\
N \\
N \\
N \\
.10\end{array}$ & $\begin{array}{r}24.0 \\
63.0 \\
22.0 \\
5.0 \\
<5.0\end{array}$ \\
\hline $\begin{array}{l}8013390 B \\
8013390 \mathrm{C} \\
80 H 3394 \\
80 H 3398 \\
80 H 3406\end{array}$ & $\begin{array}{l}N \\
N \\
N \\
N \\
N\end{array}$ & $\begin{array}{l}5 \\
N \\
N \\
N \\
N\end{array}$ & $\begin{array}{l}N \\
N \\
N \\
N \\
N\end{array}$ & $\begin{array}{r}500 \\
N \\
700 \\
200 \\
N\end{array}$ & $\begin{array}{r}20 \\
<10 \\
50 \\
20 \\
10\end{array}$ & $\begin{array}{l}N \\
N \\
N \\
N \\
N\end{array}$ & $\begin{array}{r}15 \\
N \\
N \\
N \\
N\end{array}$ & $\begin{array}{l}N \\
N \\
N \\
N \\
N\end{array}$ & $\begin{array}{r}300 \\
30 \\
200 \\
200 \\
150\end{array}$ & $\begin{array}{l}N \\
N \\
N \\
N \\
N\end{array}$ & $\begin{array}{l}.40 \\
N \\
N \\
N \\
N\end{array}$ & $\begin{array}{r}170.0 \\
<5.0 \\
78.0 \\
6.0 \\
<5.0\end{array}$ \\
\hline $\begin{array}{l}80 H 3408 \\
80 H 3409 \\
8013410 \\
8013411 \\
80 H 3413\end{array}$ & $\begin{array}{r}N \\
N \\
N \\
500 \\
N\end{array}$ & $\begin{array}{l}N \\
N \\
5 \\
N \\
N\end{array}$ & $\begin{array}{l}N \\
N \\
N \\
N \\
N\end{array}$ & $\begin{array}{r}N \\
N \\
<100 \\
N \\
N\end{array}$ & $\begin{array}{r}15 \\
10 \\
100 \\
300 \\
10\end{array}$ & $\begin{array}{l}N \\
N \\
N \\
N \\
N\end{array}$ & $\begin{array}{r}<10 \\
N \\
<10 \\
20 \\
N\end{array}$ & $\begin{array}{l}N \\
N \\
N \\
N \\
N\end{array}$ & $\begin{array}{l}200 \\
150 \\
150 \\
500 \\
500\end{array}$ & $\begin{array}{l}N \\
N \\
N \\
N \\
N\end{array}$ & $\begin{array}{l}N \\
N \\
N \\
\dot{N}^{10}\end{array}$ & $\begin{array}{r}36.0 \\
<5.0 \\
18.0 \\
1,500.0 \\
12.0\end{array}$ \\
\hline
\end{tabular}


TABLE 4--RESULTS OF ANALYSES, DETROIT MINING DISTRICT, UTAH--Continued

\begin{tabular}{|c|c|c|c|c|c|c|c|c|c|c|}
\hline Sample & $\begin{array}{l}\text { BI - PPM } \\
\text { ICP }\end{array}$ & $\begin{array}{l}\text { CD-PPM } \\
\text { ICP }\end{array}$ & $\begin{array}{l}\text { SB-PPM } \\
\text { ICP }\end{array}$ & $\begin{array}{l}Z N-P P M \\
I C P\end{array}$ & $\begin{array}{c}\mathrm{Na}-p c t . \\
s\end{array}$ & $\begin{array}{c}p-p c t . \\
s\end{array}$ & $\begin{array}{l}\text { Ga-ppm } \\
\text { s }\end{array}$ & $\begin{array}{c}\text { Ge-ppm } \\
\text { s }\end{array}$ & $\begin{array}{l}\text { HG-PPM } \\
\mathrm{CV}\end{array}$ & $\begin{array}{l}\text { F\% } \\
\text { ISE }\end{array}$ \\
\hline $\begin{array}{l}80 H 3337 A \\
80 H 3337 B \\
80 \text { H3339A } \\
80 H 3339 B \\
80 H 3340\end{array}$ & $\begin{array}{l}<2.00 \\
<2.00 \\
<2.00 \\
<2.00 \\
<2.00\end{array}$ & $\begin{array}{r}.500 \\
.400 \\
.500 \\
.700 \\
<.100\end{array}$ & $\begin{array}{r}5.00 \\
5.00 \\
<2.00 \\
<2.00 \\
<2.00\end{array}$ & $\begin{array}{r}3.00 \\
<2.00 \\
<2.00 \\
18.00 \\
<2.00\end{array}$ & $\begin{array}{l}\mathrm{N} \\
.2 \\
\mathrm{~N} \\
\mathrm{~N}\end{array}$ & $\begin{array}{l}<.2 \\
<.2 \\
.2 \\
N \\
<.2\end{array}$ & $\begin{array}{r}10 \\
10 \\
15 \\
<5 \\
5\end{array}$ & $\begin{array}{l}N \\
N \\
N \\
N \\
N\end{array}$ & $\begin{array}{l}.06 \\
.08 \\
2.08 \\
.02 \\
\mathrm{~N}\end{array}$ & $\begin{array}{r}.01 \\
<.01 \\
.03 \\
<.01 \\
<.01\end{array}$ \\
\hline $\begin{array}{l}80 H 3342 \\
80 H 3343 \\
8 D H 3344 \\
80 H 3345 \\
80 H 3346 \text { A }\end{array}$ & $\begin{array}{l}<2.00 \\
<2.00 \\
<2.00 \\
<2.00 \\
<2.00\end{array}$ & $\begin{array}{r}<.100 \\
.100 \\
.700 \\
.200 \\
<.100\end{array}$ & $\begin{array}{l}<2.00 \\
<2.00 \\
<2.00 \\
<2.00 \\
2.00\end{array}$ & $\begin{array}{r}<2.00 \\
5.00 \\
4.00 \\
9.00 \\
3.00\end{array}$ & $\begin{array}{l}N \\
N \\
N \\
<.2 \\
N\end{array}$ & $\begin{array}{l}N \\
N \\
N \\
N \\
N\end{array}$ & $\begin{array}{l}N \\
N \\
N \\
15 \\
<5\end{array}$ & $\begin{array}{l}N \\
N \\
N \\
N \\
N\end{array}$ & $\begin{array}{l}N \\
N \\
N \\
N \\
.24\end{array}$ & $\begin{array}{r}<.01 \\
<.01 \\
.02 \\
.03 \\
<.01\end{array}$ \\
\hline $\begin{array}{l}80 H 3346 B \\
80 H 3346 C \\
80 H 33460 \\
8013347 \\
80 H 3348 A\end{array}$ & $\begin{array}{r}3.00 \\
8.00 \\
<2.00 \\
<2.00 \\
<2.00\end{array}$ & $\begin{array}{r}<.100 \\
2.100 \\
.900 \\
1.100 \\
<.100\end{array}$ & $\begin{array}{r}2.00 \\
2.00 \\
2.00 \\
32.00 \\
<2.00\end{array}$ & $\begin{array}{r}<2.00 \\
6.00 \\
10.00 \\
34.00 \\
42.00\end{array}$ & $\begin{array}{c}N \\
N \\
N \\
N \\
<.2\end{array}$ & $\begin{array}{l}<.2 \\
N \\
N \\
<.2 \\
.2\end{array}$ & $\begin{array}{r}<5 \\
15 \\
N \\
15 \\
20\end{array}$ & $\begin{array}{l}N \\
N \\
N \\
N \\
N\end{array}$ & $\begin{array}{l}.04 \\
.04 \\
.52 \\
.28 \\
.02\end{array}$ & $\begin{array}{r}.01 \\
.03 \\
<.01 \\
.01 \\
.02\end{array}$ \\
\hline $\begin{array}{l}80 H 3348 B \\
80 H 3349 \\
80 H 3350 A \\
80 H 3350 B \\
80 H 3350 C\end{array}$ & $\begin{array}{l}<2.00 \\
<2.00 \\
<2.00 \\
<2.00 \\
11.00\end{array}$ & $\begin{array}{l}.600 \\
.500 \\
.400 \\
.300 \\
1.600\end{array}$ & $\begin{array}{r}<2.00 \\
24.00 \\
<2.00 \\
3.00 \\
42.00\end{array}$ & $\begin{array}{r}86.00 \\
51.00 \\
25.00 \\
120.00 \\
120.00\end{array}$ & $\begin{array}{l}<.2 \\
N \\
N \\
N \\
N\end{array}$ & $\begin{array}{c}.5 \\
N \\
N \\
2.0\end{array}$ & $\begin{array}{c}10 \\
N \\
15 \\
10 \\
10\end{array}$ & $\begin{array}{l}N \\
N \\
N \\
N \\
N\end{array}$ & $\begin{array}{r}.08 \\
.06 \\
.04 \\
1.10 \\
.10\end{array}$ & $\begin{array}{r}.07 \\
<.01 \\
.02 \\
.06 \\
.02\end{array}$ \\
\hline $\begin{array}{l}80 H 3351 \\
80 H 3353 \\
80 H 3354 \\
80 H 3355 \\
8013363\end{array}$ & $\begin{array}{l}<2.00 \\
<2.00 \\
<2.00 \\
<2.00 \\
<2.00\end{array}$ & $\begin{array}{r}.500 \\
.100 \\
<.100 \\
.500 \\
<.100\end{array}$ & $\begin{array}{r}23.00 \\
6.00 \\
<2.00 \\
<2.00 \\
<2.00\end{array}$ & $\begin{array}{r}61.00 \\
<2.00 \\
<2.00 \\
8.00 \\
<2.00\end{array}$ & $\begin{array}{l}N \\
N \\
N \\
N \\
.3\end{array}$ & $\begin{array}{l}<.2 \\
<.2 \\
.2 \\
.3 \\
N\end{array}$ & $\begin{array}{r}5 \\
<5 \\
30 \\
15 \\
100\end{array}$ & $\begin{array}{l}N \\
N \\
N \\
N \\
N\end{array}$ & $\begin{array}{l}1.60 \\
.02 \\
.02 \\
N \\
.02\end{array}$ & $\begin{array}{l}<.01 \\
<.01 \\
.05 \\
.03 \\
<.01\end{array}$ \\
\hline $\begin{array}{l}80 H 3369 \\
80 \text { H3377 } \\
80 \text { H3378 } \\
80 \text { H3379 } \\
80 H 3380\end{array}$ & $\begin{array}{r}<2.00 \\
<2.00 \\
<2.00 \\
3.00 \\
2.00\end{array}$ & $\begin{array}{r}.100 \\
<.100 \\
4.900 \\
.300 \\
.500\end{array}$ & $\begin{array}{r}<2.00 \\
<2.00 \\
18.00 \\
2.00 \\
7.00\end{array}$ & $\begin{array}{r}<2.00 \\
3.00 \\
24.00 \\
<2.00 \\
27.00\end{array}$ & $\begin{array}{l}N \\
N \\
N \\
N \\
N\end{array}$ & $\begin{array}{l}<.2 \\
<.2 \\
N \\
<.2 \\
N\end{array}$ & $\begin{array}{l}N \\
<5 \\
10 \\
15 \\
<5\end{array}$ & $\begin{array}{l}N \\
N \\
N \\
N \\
N\end{array}$ & $\begin{array}{l}.02 \\
N \\
.78 \\
.04 \\
.08\end{array}$ & $\begin{array}{l}N \\
N \\
N \\
N \\
N\end{array}$ \\
\hline $\begin{array}{l}80 H 3381 \\
80 H 3385 \\
80 H 3386 \\
80 H 3388 \\
8013390 A\end{array}$ & $\begin{array}{l}<2.00 \\
<2.00 \\
<2.00 \\
<2.00 \\
<2.00\end{array}$ & $\begin{array}{r}3.600 \\
.400 \\
.600 \\
.200 \\
.200\end{array}$ & $\begin{array}{r}<2.00 \\
2.00 \\
9.00 \\
<2.00 \\
3.00\end{array}$ & $\begin{array}{r}6.00 \\
6.00 \\
26.00 \\
6.00 \\
2.00\end{array}$ & $\begin{array}{l}N \\
N \\
N \\
N \\
N\end{array}$ & $\begin{array}{l}N \\
<.2 \\
N \\
N \\
N\end{array}$ & $\begin{array}{r}5 \\
5 \\
<5 \\
N \\
N\end{array}$ & $\begin{array}{l}N \\
N \\
N \\
N \\
N\end{array}$ & $\begin{array}{l}N \\
N \\
.10 \\
N \\
N\end{array}$ & $\begin{array}{l}N \\
N \\
N \\
N \\
.10\end{array}$ \\
\hline $\begin{array}{l}8013390 \mathrm{~B} \\
8013390 \mathrm{C} \\
80 \mathrm{H} 3394 \\
80 \mathrm{H} 3398 \\
80 \mathrm{H} 3406\end{array}$ & $\begin{array}{l}20.00 \\
<2.00 \\
12.00 \\
<2.00 \\
<2.00\end{array}$ & $\begin{array}{r}.900 \\
<.100 \\
1.200 \\
1.800 \\
.100\end{array}$ & $\begin{array}{r}53.00 \\
2.00 \\
10.00 \\
<2.00 \\
<2.00\end{array}$ & $\begin{array}{r}7.00 \\
<2.00 \\
9.00 \\
110.00 \\
3.00\end{array}$ & $\begin{array}{l}N \\
N \\
N \\
<.2 \\
N\end{array}$ & $\begin{array}{l}<.2 \\
N \\
.2 \\
N\end{array}$ & $\begin{array}{r}<5 \\
N \\
15 \\
7 \\
<5\end{array}$ & $\begin{array}{l}N \\
N \\
N \\
N \\
N\end{array}$ & $\begin{array}{l}\dot{N}^{14} \\
.04 \\
N \\
N\end{array}$ & $\begin{array}{l}.40 \\
N \\
N \\
N \\
N\end{array}$ \\
\hline $\begin{array}{l}80 H 3408 \\
80 H 3409 \\
8013410 \\
8013411 \\
80 H 3413\end{array}$ & $\begin{array}{r}3.00 \\
<2.00 \\
<2.00 \\
510.00 \\
<2.00\end{array}$ & $\begin{array}{l}.400 \\
.100 \\
<.100 \\
2.900 \\
<.100\end{array}$ & $\begin{array}{r}2.00 \\
<2.00 \\
<2.00 \\
420.00 \\
9.00\end{array}$ & $\begin{array}{r}14.00 \\
3.00 \\
<2.00 \\
24.00 \\
<2.00\end{array}$ & $\begin{array}{l}N \\
N \\
<.2 \\
N \\
N\end{array}$ & $\begin{array}{l}N \\
<.2 \\
<.2 \\
N \\
<.2\end{array}$ & $\begin{array}{r}<5 \\
N \\
15 \\
20 \\
N\end{array}$ & $\begin{array}{l}N \\
N \\
N \\
N \\
N\end{array}$ & $\begin{array}{l}.02 \\
.04 \\
.18 \\
.80 \\
.02\end{array}$ & $\begin{array}{l}N \\
N \\
N \\
.10 \\
N\end{array}$ \\
\hline
\end{tabular}


TABLE 4--RESULTS OF ANALYSES, DETROIT MINING DISTRICT, UTAH--Continued

\begin{tabular}{|c|c|c|c|c|c|c|c|c|c|c|c|}
\hline Sample & Latitude & Long & i tude & $\begin{array}{c}\text { Fe-pct. } \\
\text { s }\end{array}$ & $\begin{array}{c}\text { Mg-pct. } \\
\text { s }\end{array}$ & $\begin{array}{c}\text { Ca-pct. } \\
s\end{array}$ & $\begin{array}{c}\text { Ti-pct } \\
\text { s }\end{array}$ & $\begin{array}{l}\text { Mn-ppm } \\
s\end{array}$ & $\begin{array}{c}\text { Ag-ppm } \\
\text { s }\end{array}$ & $\begin{array}{c}\text { As-ppm } \\
\text { s }\end{array}$ & $\begin{array}{c}\text { Au-ppm } \\
\text { s }\end{array}$ \\
\hline $\begin{array}{l}\text { 9dh3467a } \\
9 \mathrm{dh} 3467 \mathrm{~b} \\
\text { 9dh3468b } \\
\text { 9dh3468c } \\
\text { 9dh3469 }\end{array}$ & $\begin{array}{lll}39 & 31 & 36 \\
39 & 31 & 36 \\
39 & 31 & 36 \\
39 & 31 & 36 \\
39 & 31 & 36\end{array}$ & $\begin{array}{l}113 \\
113 \\
113 \\
113 \\
113\end{array}$ & $\begin{array}{ll}0 & 32 \\
0 & 32 \\
0 & 32 \\
0 & 32 \\
0 & 32\end{array}$ & $\begin{array}{r}.70 \\
5.00 \\
10.00 \\
5.00 \\
.70\end{array}$ & $\begin{array}{r}<.02 \\
.20 \\
1.00 \\
7.00 \\
3.00\end{array}$ & $\begin{array}{r}<.05 \\
.10 \\
5.00 \\
1.00 \\
.07\end{array}$ & $\begin{array}{l}.150 \\
.500 \\
.050 \\
.150 \\
.200\end{array}$ & $\begin{array}{r}20 \\
50 \\
>5,000 \\
2,000 \\
300\end{array}$ & $\begin{array}{l}N \\
N \\
N \\
N \\
N\end{array}$ & $\begin{array}{l}N \\
N \\
N \\
N \\
N\end{array}$ & $\begin{array}{l}\mathbf{N} \\
\mathbf{N} \\
\mathbf{N} \\
\mathbf{N} \\
\mathbf{N}\end{array}$ \\
\hline $\begin{array}{l}9 d h 3470 a \\
9 d h 3470 b \\
9 d h 3470 c \\
9 d h 3471 \\
9 d h 3472 a\end{array}$ & $\begin{array}{lll}39 & 31 & 36 \\
39 & 31 & 36 \\
39 & 31 & 36 \\
39 & 31 & 36 \\
39 & 31 & 36\end{array}$ & $\begin{array}{l}113 \\
113 \\
113 \\
113 \\
113\end{array}$ & $\begin{array}{ll}0 & 32 \\
0 & 32 \\
0 & 32 \\
0 & 32 \\
0 & 32\end{array}$ & $\begin{array}{l}5.00 \\
<.05 \\
2.00 \\
5.00 \\
5.00\end{array}$ & $\begin{array}{l}<.02 \\
<.02 \\
<.02 \\
<.02 \\
.70\end{array}$ & $\begin{array}{l}.05 \\
<.05 \\
<.05 \\
<.05 \\
.07\end{array}$ & $\begin{array}{l}.100 \\
.200 \\
.200 \\
.150 \\
.500\end{array}$ & $\begin{array}{r}10 \\
50 \\
70 \\
30 \\
500\end{array}$ & $\begin{array}{l}N \\
N \\
N \\
N \\
N\end{array}$ & $\begin{array}{r}<200 \\
N \\
N \\
N \\
N\end{array}$ & $\begin{array}{r}<10 \\
N \\
N \\
N \\
N\end{array}$ \\
\hline $\begin{array}{l}9 \mathrm{dh} 3472 b \\
9 \mathrm{dh} 3473 \mathrm{a} \\
9 \mathrm{dh} 3473 \mathrm{~b} \\
9 \mathrm{dh} 3473 \mathrm{c} \\
9 \mathrm{dh} 3473 \mathrm{~d}\end{array}$ & $\begin{array}{lll}39 & 31 & 36 \\
39 & 31 & 36 \\
39 & 31 & 36 \\
39 & 31 & 36 \\
39 & 31 & 36\end{array}$ & $\begin{array}{l}113 \\
113 \\
113 \\
113 \\
113\end{array}$ & $\begin{array}{ll}0 & 32 \\
0 & 32 \\
0 & 32 \\
0 & 32 \\
0 & 32\end{array}$ & $\begin{array}{r}1.50 \\
5.00 \\
3.00 \\
.10 \\
.10\end{array}$ & $\begin{array}{r}.50 \\
.20 \\
<.02 \\
<.02 \\
<.02\end{array}$ & $\begin{array}{r}20.00 \\
.07 \\
.05 \\
.05 \\
.05\end{array}$ & $\begin{array}{l}.150 \\
.200 \\
.200 \\
.150 \\
.300\end{array}$ & $\begin{array}{r}1,000 \\
200 \\
30 \\
20 \\
<10\end{array}$ & $\begin{array}{l}N \\
N \\
N \\
N \\
N\end{array}$ & $\begin{array}{r}N \\
<200 \\
N \\
N \\
N\end{array}$ & $\begin{array}{l}N \\
N \\
N \\
N \\
N\end{array}$ \\
\hline $\begin{array}{l}9 \mathrm{dh} 3473 \mathrm{e} \\
9 \mathrm{dh} 3473 \mathrm{f} \\
9 \mathrm{dh} 3473 \mathrm{~g} \\
9 \mathrm{dh} 3474 \mathrm{~b} \\
9 \mathrm{dh} 3474 \mathrm{c}\end{array}$ & $\begin{array}{lll}39 & 31 & 36 \\
39 & 31 & 36 \\
39 & 31 & 36 \\
39 & 31 & 36 \\
39 & 31 & 36\end{array}$ & $\begin{array}{l}113 \\
113 \\
113 \\
113 \\
113\end{array}$ & $\begin{array}{ll}0 & 32 \\
0 & 32 \\
0 & 32 \\
0 & 32 \\
0 & 32\end{array}$ & $\begin{array}{r}15.15 \\
.00 \\
.50 \\
20.00 \\
.70\end{array}$ & $\begin{array}{r}<.02 \\
.02 \\
<.02 \\
.20 \\
.50\end{array}$ & $\begin{array}{r}<.05 \\
.05 \\
.10 \\
.30 \\
<.05\end{array}$ & $\begin{array}{l}.500 \\
.150 \\
.100 \\
.070 \\
.200\end{array}$ & $\begin{array}{r}10 \\
50 \\
15 \\
>5,000 \\
50\end{array}$ & $\begin{array}{l}N \\
N \\
N \\
N \\
N\end{array}$ & $\begin{array}{r}N \\
1,500 \\
N \\
<200 \\
N\end{array}$ & $\begin{array}{l}N \\
N \\
N \\
N \\
N\end{array}$ \\
\hline $\begin{array}{l}\text { 9dh3476 } \\
\text { 9dh3477 } \\
\text { 9dh3478 } \\
\text { 9dh3479 } \\
\text { 9dh3480 }\end{array}$ & $\begin{array}{lll}39 & 31 & 36 \\
39 & 31 & 36 \\
39 & 31 & 36 \\
39 & 31 & 36 \\
39 & 31 & 36\end{array}$ & $\begin{array}{l}113 \\
113 \\
113 \\
113 \\
113\end{array}$ & $\begin{array}{ll}0 & 32 \\
0 & 32 \\
0 & 32 \\
0 & 32 \\
0 & 32\end{array}$ & $\begin{array}{r}5.00 \\
10.00 \\
10.00 \\
20.00 \\
5.00\end{array}$ & $\begin{array}{l}.50 \\
.20 \\
.70 \\
.30 \\
.50\end{array}$ & $\begin{array}{l}.10 \\
.07 \\
1.00 \\
.20 \\
.05\end{array}$ & $\begin{array}{l}.200 \\
.100 \\
.100 \\
.050 \\
.200\end{array}$ & $\begin{array}{r}200 \\
100 \\
2,000 \\
700 \\
20\end{array}$ & $\begin{array}{l}N \\
N \\
N \\
N \\
N\end{array}$ & $\begin{array}{r}N \\
N \\
N \\
500 \\
N\end{array}$ & $\begin{array}{l}N \\
N \\
N \\
N \\
N\end{array}$ \\
\hline $\begin{array}{l}9 \mathrm{dh} 3481 \\
9 \mathrm{dh} 3482 \\
9 \mathrm{dh} 3483 \\
9 \mathrm{dh} 3484 \\
9 \mathrm{dh} 3485\end{array}$ & $\begin{array}{lll}39 & 31 & 36 \\
39 & 31 & 36 \\
39 & 31 & 36 \\
39 & 31 & 36 \\
39 & 31 & 36\end{array}$ & $\begin{array}{l}113 \\
113 \\
113 \\
113 \\
113\end{array}$ & $\begin{array}{ll}0 & 32 \\
0 & 32 \\
0 & 32 \\
0 & 32 \\
0 & 32\end{array}$ & $\begin{array}{r}1.00 \\
.05 \\
.30 \\
3.00 \\
3.00\end{array}$ & $\begin{array}{l}<.02 \\
<.02 \\
<.02 \\
.20 \\
.10\end{array}$ & $\begin{array}{r}<.05 \\
<.05 \\
<.05 \\
>20.00 \\
.30\end{array}$ & $\begin{array}{l}.150 \\
.150 \\
.200 \\
.005 \\
.070\end{array}$ & $\begin{array}{r}10 \\
30 \\
10 \\
2,000 \\
>5,000\end{array}$ & $\begin{array}{l}N \\
N \\
N \\
N \\
N\end{array}$ & $\begin{array}{r}N \\
N \\
N \\
N \\
1,000\end{array}$ & $\begin{array}{l}\mathbf{N} \\
\mathbf{N} \\
\mathbf{N} \\
\mathbf{N} \\
\mathbf{N}\end{array}$ \\
\hline $\begin{array}{l}9 \mathrm{dh} 3486 \\
9 \mathrm{dh} 3487 \\
9 \mathrm{dh} 3488 \mathrm{~b} \\
9 \mathrm{dh} 3488 \mathrm{e} \\
9 \mathrm{dh} 3488 \mathrm{f}\end{array}$ & $\begin{array}{lll}39 & 31 & 36 \\
39 & 31 & 36 \\
39 & 31 & 39 \\
39 & 31 & 39 \\
39 & 31 & 39\end{array}$ & $\begin{array}{l}113 \\
113 \\
113 \\
113 \\
113\end{array}$ & $\begin{array}{ll}0 & 32 \\
0 & 32 \\
0 & 25 \\
0 & 25 \\
0 & 25\end{array}$ & $\begin{array}{r}5.00 \\
.70 \\
2.00 \\
7.00 \\
.20\end{array}$ & $\begin{array}{l}<.02 \\
.50 \\
<.02 \\
<.02 \\
<.02\end{array}$ & $\begin{array}{r}.05 \\
.20 \\
<.05 \\
<.05 \\
.05\end{array}$ & $\begin{array}{l}.200 \\
.200 \\
.100 \\
.500 \\
.200\end{array}$ & $\begin{array}{r}70 \\
100 \\
100 \\
10 \\
<10\end{array}$ & $\begin{array}{l}N \\
N \\
N \\
N \\
N\end{array}$ & $\begin{array}{l}N \\
N \\
N \\
N \\
N\end{array}$ & $\begin{array}{r}N \\
N \\
N \\
20 \\
15\end{array}$ \\
\hline $\begin{array}{l}\text { 9dh3488g } \\
\text { 9dh3490 } \\
\text { 9dh3492 } \\
\text { 9dh3493a } \\
\text { 9dh3493b }\end{array}$ & $\begin{array}{lll}39 & 31 & 39 \\
39 & 31 & 39 \\
39 & 31 & 39 \\
39 & 31 & 39 \\
39 & 31 & 39\end{array}$ & $\begin{array}{l}113 \\
113 \\
113 \\
113 \\
113\end{array}$ & $\begin{array}{ll}0 & 25 \\
0 & 25 \\
0 & 25 \\
0 & 25 \\
0 & 25\end{array}$ & $\begin{array}{r}1.00 \\
3.00 \\
3.00 \\
.02 \\
2.00\end{array}$ & $\begin{array}{l}<.02 \\
<.02 \\
.02 \\
.70 \\
.50\end{array}$ & $\begin{array}{r}.05 \\
<.05 \\
.05 \\
.10 \\
.07\end{array}$ & $\begin{array}{l}.200 \\
.200 \\
.300 \\
.300 \\
.300\end{array}$ & $\begin{array}{r}200 \\
150 \\
10 \\
1,000 \\
30\end{array}$ & $\begin{array}{l}N \\
N \\
N \\
N \\
N\end{array}$ & $\begin{array}{l}N \\
N \\
N \\
N \\
N\end{array}$ & $\begin{array}{l}N \\
N \\
N \\
N \\
N\end{array}$ \\
\hline $\begin{array}{l}\text { 9dh3493c } \\
\text { 9dh3493d } \\
\text { 9dh3493e } \\
\text { 9dh3493f } \\
\text { 9dh3493g }\end{array}$ & $\begin{array}{lll}39 & 31 & 39 \\
39 & 31 & 39 \\
39 & 31 & 39 \\
39 & 31 & 39 \\
39 & 31 & 39\end{array}$ & $\begin{array}{l}113 \\
113 \\
113 \\
113 \\
113\end{array}$ & $\begin{array}{ll}0 & 25 \\
0 & 25 \\
0 & 25 \\
0 & 25 \\
0 & 25\end{array}$ & $\begin{array}{r}1.00 \\
.70 \\
<.05 \\
.50 \\
.07\end{array}$ & $\begin{array}{l}.02 \\
<.02 \\
<.02 \\
<.02 \\
<.02\end{array}$ & $\begin{array}{l}<.05 \\
<.05 \\
<.05 \\
<.05 \\
<.05\end{array}$ & $\begin{array}{l}.200 \\
.150 \\
.150 \\
.200 \\
.200\end{array}$ & $\begin{array}{r}100 \\
500 \\
20 \\
<10 \\
10\end{array}$ & $\begin{array}{l}N \\
N \\
N \\
N \\
N\end{array}$ & $\begin{array}{l}N \\
N \\
N \\
N \\
N\end{array}$ & $\begin{array}{l}N \\
N \\
N \\
N \\
N\end{array}$ \\
\hline
\end{tabular}


TABLE 4--RESULTS OF ANALYSES, DETROIT MINING DISTRICT, UTAH--Continued

\begin{tabular}{|c|c|c|c|c|c|c|c|c|c|c|c|c|c|}
\hline Sample & $\begin{array}{c}\text { B-ppm } \\
\text { s }\end{array}$ & $\begin{array}{c}\text { Ba-ppm } \\
s\end{array}$ & $\begin{array}{c}\text { Be-ppm } \\
s\end{array}$ & $\begin{array}{c}\text { Bi-ppm } \\
\text { s }\end{array}$ & $\begin{array}{c}\text { Cd-ppm } \\
s\end{array}$ & $\begin{array}{c}\text { Co-ppm } \\
s\end{array}$ & $\begin{array}{c}\text { Cr-ppm } \\
s\end{array}$ & $\begin{array}{c}\text { Cu-ppm } \\
s\end{array}$ & $\begin{array}{c}\text { La-ppm } \\
s\end{array}$ & $\begin{array}{l}\text { Mo-ppm } \\
\mathrm{s}\end{array}$ & $\begin{array}{c}\text { Nb-ppm } \\
s\end{array}$ & $\begin{array}{c}\mathrm{Ni} \text {-ppm } \\
s\end{array}$ & $\begin{array}{l}\mathrm{Pb}-\mathrm{ppm} \\
\quad s\end{array}$ \\
\hline $\begin{array}{l}9 \mathrm{dh} 3467 \mathrm{a} \\
9 \mathrm{dh} 3467 \mathrm{~b} \\
9 \mathrm{dh} 3468 \mathrm{~b} \\
9 \mathrm{dh} 3468 \mathrm{c} \\
9 \mathrm{dh} 3469\end{array}$ & $\begin{array}{r}10 \\
20 \\
N \\
<10 \\
50\end{array}$ & $\begin{array}{r}100 \\
500 \\
5,000 \\
30 \\
1,000\end{array}$ & $\begin{array}{r}N \\
<1.0 \\
1.0 \\
<1.0 \\
2.0\end{array}$ & $\begin{array}{l}N \\
N \\
N \\
N \\
N\end{array}$ & $\begin{array}{l}N \\
N \\
N \\
N \\
N\end{array}$ & $\begin{array}{r}N \\
<10 \\
10 \\
N \\
<10\end{array}$ & $\begin{array}{r}<10 \\
150 \\
<10 \\
30 \\
50\end{array}$ & $\begin{array}{r}20 \\
50 \\
100 \\
30 \\
<5\end{array}$ & $\begin{array}{r}N \\
50 \\
N \\
<50 \\
50\end{array}$ & $\begin{array}{r}N \\
<5 \\
70 \\
N \\
N\end{array}$ & $\begin{array}{l}\mathrm{N} \\
\mathrm{N} \\
\mathrm{N} \\
\mathrm{N} \\
\mathrm{N}\end{array}$ & $\begin{array}{r}<5 \\
7 \\
10 \\
<5 \\
<5\end{array}$ & $\begin{array}{r}<10 \\
100 \\
150 \\
20 \\
<10\end{array}$ \\
\hline $\begin{array}{l}9 \mathrm{dh} 3470 \mathrm{a} \\
9 \mathrm{dh} 3470 \mathrm{~b} \\
9 \mathrm{dh} 3470 \mathrm{c} \\
9 \mathrm{dh} 3471 \\
9 \mathrm{dh} 3472 \mathrm{a}\end{array}$ & $\begin{array}{r}<10 \\
10 \\
<10 \\
<10 \\
50\end{array}$ & $\begin{array}{r}200 \\
30 \\
200 \\
500 \\
500\end{array}$ & $\begin{array}{c}N \\
N \\
N \\
N \\
5.0\end{array}$ & $\begin{array}{l}N \\
N \\
N \\
N \\
N\end{array}$ & $\begin{array}{l}N \\
N \\
N \\
N \\
N\end{array}$ & $\begin{array}{r}<10 \\
N \\
N \\
<10 \\
N\end{array}$ & $\begin{array}{r}70 \\
<10 \\
30 \\
<10 \\
50\end{array}$ & $\begin{array}{r}200 \\
<5 \\
70 \\
300 \\
7\end{array}$ & $\begin{array}{r}50 \\
N \\
N \\
N \\
50\end{array}$ & $\begin{array}{r}<5 \\
N \\
N \\
N \\
N\end{array}$ & $\begin{array}{r}N \\
N \\
N \\
N \\
<20\end{array}$ & $\begin{array}{l}N \\
<5 \\
<5 \\
<5 \\
<5\end{array}$ & $\begin{array}{r}500 \\
N \\
50 \\
50 \\
100\end{array}$ \\
\hline $\begin{array}{l}\text { 9dh3472b } \\
9 \mathrm{dh} 3473 \mathrm{a} \\
9 \mathrm{dh} 3473 \mathrm{~b} \\
9 \mathrm{dh} 3473 \mathrm{c} \\
9 \mathrm{dh} 3473 \mathrm{~d}\end{array}$ & $\begin{array}{r}30 \\
15 \\
<10 \\
10 \\
10\end{array}$ & $\begin{array}{r}200 \\
70 \\
150 \\
100 \\
300\end{array}$ & $\begin{array}{r}1.0 \\
1.5 \\
<1.0 \\
N \\
<1.0\end{array}$ & $\begin{array}{l}N \\
N \\
N \\
N \\
N\end{array}$ & $\begin{array}{l}N \\
N \\
N \\
N \\
N\end{array}$ & $\begin{array}{r}<10 \\
20 \\
<10 \\
N \\
N\end{array}$ & $\begin{array}{r}10 \\
20 \\
10 \\
10 \\
150\end{array}$ & $\begin{array}{r}10 \\
200 \\
70 \\
5 \\
7\end{array}$ & $\begin{array}{r}N \\
<50 \\
N \\
<50 \\
70\end{array}$ & $\begin{array}{l}N \\
N \\
N \\
N \\
N\end{array}$ & $\begin{array}{l}N \\
N \\
N \\
N \\
N\end{array}$ & $\begin{array}{r}5 \\
30 \\
<5 \\
N \\
<5\end{array}$ & $\begin{array}{r}10 \\
150 \\
10 \\
30 \\
150\end{array}$ \\
\hline $\begin{array}{l}\text { 9dh3473e } \\
9 \mathrm{dh} 3473 \mathrm{f} \\
9 \mathrm{dh} 3473 \mathrm{~g} \\
9 \mathrm{dh} 3474 \mathrm{~b} \\
9 \mathrm{dh} 3474 \mathrm{c}\end{array}$ & $\begin{array}{r}10 \\
N \\
10 \\
N \\
100\end{array}$ & $\begin{array}{r}100 \\
100 \\
70 \\
2,000 \\
500\end{array}$ & $\begin{array}{l}N \\
2.0 \\
N \\
5.0 \\
2.0\end{array}$ & $\begin{array}{l}N \\
N \\
N \\
N \\
N\end{array}$ & $\begin{array}{l}\mathrm{N} \\
\mathrm{N} \\
\mathrm{N} \\
\mathrm{N} \\
\mathrm{N}\end{array}$ & $\begin{array}{r}N \\
15 \\
N \\
30 \\
N\end{array}$ & $\begin{array}{r}10 \\
70 \\
<10 \\
<10 \\
50\end{array}$ & $\begin{array}{r}10 \\
300 \\
70 \\
300 \\
7\end{array}$ & $\begin{array}{r}N \\
N \\
N \\
N \\
<50\end{array}$ & $\begin{array}{l}N \\
5 \\
N \\
N \\
N\end{array}$ & $\begin{array}{l}N \\
N \\
N \\
N \\
N\end{array}$ & $\begin{array}{r}<5 \\
20 \\
<5 \\
100 \\
10\end{array}$ & $\begin{array}{r}N \\
50 \\
N \\
200 \\
30\end{array}$ \\
\hline $\begin{array}{l}\text { 9dh3476 } \\
\text { 9dh3477 } \\
9 \mathrm{dh} 3478 \\
9 \mathrm{dh} 3479 \\
9 \mathrm{dh} 3480\end{array}$ & $\begin{array}{r}70 \\
20 \\
20 \\
N \\
50\end{array}$ & $\begin{array}{r}1,000 \\
100 \\
300 \\
50 \\
300\end{array}$ & $\begin{array}{r}2.0 \\
1.5 \\
2.0 \\
3.0 \\
<1.0\end{array}$ & $\begin{array}{l}N \\
N \\
N \\
N \\
N\end{array}$ & $\begin{array}{l}\text { N } \\
N \\
N \\
N \\
N\end{array}$ & $\begin{array}{r}<10 \\
<10 \\
10 \\
20 \\
N\end{array}$ & $\begin{array}{r}30 \\
10 \\
10 \\
<10 \\
30\end{array}$ & $\begin{array}{l}50 \\
20 \\
30 \\
70 \\
70\end{array}$ & $\begin{array}{r}<50 \\
N \\
<50 \\
N \\
<50\end{array}$ & $\begin{array}{l}\mathbf{N} \\
\mathbf{N} \\
\mathbf{N} \\
\mathbf{N} \\
\mathbf{N}\end{array}$ & $\begin{array}{l}N \\
N \\
N \\
N \\
N\end{array}$ & $\begin{array}{r}7 \\
15 \\
30 \\
20 \\
<5\end{array}$ & $\begin{array}{r}150 \\
200 \\
200 \\
300 \\
20\end{array}$ \\
\hline $\begin{array}{l}\text { 9dh3481 } \\
9 \mathrm{dh} 3482 \\
9 \mathrm{dh} 3483 \\
9 \mathrm{dh} 3484 \\
9 \mathrm{dh} 3485\end{array}$ & $\begin{array}{r}10 \\
10 \\
10 \\
<10 \\
10\end{array}$ & $\begin{array}{r}200 \\
200 \\
50 \\
300 \\
3,000\end{array}$ & $\begin{array}{c}N \\
N \\
N \\
N \\
5.0\end{array}$ & $\begin{array}{r}N \\
N \\
N \\
N \\
10\end{array}$ & $\begin{array}{l}N \\
N \\
N \\
N \\
N\end{array}$ & $\begin{array}{r}N \\
N \\
N \\
15 \\
150\end{array}$ & $\begin{array}{r}N \\
15 \\
<10 \\
N \\
<10\end{array}$ & $\begin{array}{r}10 \\
<5 \\
5 \\
50 \\
300\end{array}$ & $\begin{array}{l}\mathbf{N} \\
\mathbf{N} \\
\mathbf{N} \\
\mathbf{N} \\
\mathbf{N}\end{array}$ & $\begin{array}{l}N \\
N \\
N \\
<5 \\
15\end{array}$ & $\begin{array}{l}N \\
N \\
N \\
N \\
N\end{array}$ & $\begin{array}{c}N \\
N \\
N \\
10 \\
70\end{array}$ & $\begin{array}{r}<10 \\
30 \\
<10 \\
1,000 \\
500\end{array}$ \\
\hline $\begin{array}{l}\text { 9dh3486 } \\
\text { 9dh3487 } \\
\text { 9dh3488b } \\
\text { 9dh3488e } \\
\text { 9dh3488f }\end{array}$ & $\begin{array}{l}10 \\
50 \\
10 \\
20 \\
30\end{array}$ & $\begin{array}{r}500 \\
700 \\
20 \\
50 \\
100\end{array}$ & $\begin{array}{c}<1.0 \\
2.0 \\
N \\
N \\
N\end{array}$ & $\begin{array}{l}N \\
N \\
N \\
N \\
N\end{array}$ & $\begin{array}{l}N \\
N \\
N \\
N \\
N\end{array}$ & $\begin{array}{r}10 \\
N \\
N \\
N \\
N\end{array}$ & $\begin{array}{r}30 \\
30 \\
10 \\
200 \\
70\end{array}$ & $\begin{array}{l}30 \\
10 \\
<5 \\
15 \\
<5\end{array}$ & $\begin{array}{r}<50 \\
N \\
N \\
<50 \\
N\end{array}$ & $\begin{array}{l}N \\
N \\
N \\
N \\
N\end{array}$ & $\begin{array}{r}N \\
N \\
N \\
20 \\
N\end{array}$ & $\begin{array}{l}<5 \\
N \\
N \\
N \\
N\end{array}$ & $\begin{array}{l}200 \\
100 \\
150 \\
500 \\
100\end{array}$ \\
\hline $\begin{array}{l}9 \mathrm{dh} 3488 \mathrm{~g} \\
9 \mathrm{dh} 3490 \\
9 \mathrm{dh} 3492 \\
9 \mathrm{dh} 3493 \mathrm{a} \\
9 \mathrm{dh} 3493 \mathrm{~b}\end{array}$ & $\begin{array}{r}15 \\
10 \\
10 \\
50 \\
100\end{array}$ & $\begin{array}{r}200 \\
50 \\
300 \\
1,000 \\
1,000\end{array}$ & $\begin{array}{c}N \\
<1.0 \\
N \\
<1.0 \\
2.0\end{array}$ & $\begin{array}{l}N \\
N \\
N \\
N \\
N\end{array}$ & $\begin{array}{l}N \\
N \\
N \\
N \\
N\end{array}$ & $\begin{array}{r}N \\
N \\
<10 \\
<10 \\
<10\end{array}$ & $\begin{array}{r}70 \\
30 \\
100 \\
20 \\
150\end{array}$ & $\begin{array}{r}7 \\
20 \\
30 \\
<5 \\
30\end{array}$ & $\begin{array}{r}N \\
<50 \\
<50 \\
50 \\
50\end{array}$ & $\begin{array}{l}N \\
N \\
N \\
N \\
N\end{array}$ & $\begin{array}{l}\text { N } \\
N \\
N \\
N \\
N\end{array}$ & $\begin{array}{l}<5 \\
<5 \\
10 \\
<5 \\
20\end{array}$ & $\begin{array}{r}300 \\
30 \\
20 \\
100 \\
150\end{array}$ \\
\hline $\begin{array}{l}9 \mathrm{dh} 3493 \mathrm{c} \\
9 \mathrm{dh} 3493 \mathrm{~d} \\
9 \mathrm{dh} 3493 \mathrm{e} \\
9 \mathrm{dh} 3493 \mathrm{f} \\
9 \mathrm{dh} 3493 \mathrm{~g}\end{array}$ & $\begin{array}{l}10 \\
10 \\
10 \\
10 \\
N\end{array}$ & $\begin{array}{r}100 \\
70 \\
50 \\
700 \\
700\end{array}$ & $\begin{array}{c}<1.0 \\
N \\
N \\
N \\
N\end{array}$ & $\begin{array}{l}\mathrm{N} \\
\mathrm{N} \\
\mathrm{N} \\
\mathrm{N} \\
\mathrm{N}\end{array}$ & $\begin{array}{l}N \\
N \\
N \\
N \\
N\end{array}$ & $\begin{array}{r}<10 \\
N \\
N \\
N \\
N\end{array}$ & $\begin{array}{l}30 \\
20 \\
30 \\
20 \\
10\end{array}$ & $\begin{array}{r}5 \\
10 \\
<5 \\
7 \\
5\end{array}$ & $\begin{array}{r}<50 \\
N \\
N \\
N \\
N\end{array}$ & $\begin{array}{l}\mathrm{N} \\
\mathrm{N} \\
\mathrm{N} \\
\mathrm{N} \\
\mathrm{N}\end{array}$ & $\begin{array}{l}N \\
N \\
N \\
N \\
N\end{array}$ & $\begin{array}{r}7 \\
5 \\
<5 \\
<5 \\
N\end{array}$ & $\begin{array}{r}<10 \\
<10 \\
<10 \\
<10 \\
20\end{array}$ \\
\hline
\end{tabular}


TABLE 4--RESULTS OF ANALYSES, DETROIT MINING DISTRICT, UTAH--Continued

\begin{tabular}{|c|c|c|c|c|c|c|c|c|c|c|c|c|}
\hline Sample & $\begin{array}{c}\text { sb-ppm } \\
s\end{array}$ & $\begin{array}{c}\text { Sc-ppm } \\
s\end{array}$ & $\begin{array}{c}\text { sn-ppm } \\
s\end{array}$ & $\begin{array}{c}\text { Sr-ppm } \\
s\end{array}$ & $\begin{array}{c}v-p p m \\
s\end{array}$ & $\begin{array}{c}W \text {-ppm } \\
s\end{array}$ & $\begin{array}{c}Y-p p m \\
s\end{array}$ & $\begin{array}{c}\text { Zn-ppm } \\
s\end{array}$ & $\begin{array}{c}\text { Zr-ppm } \\
s\end{array}$ & $\begin{array}{c}\text { Th-ppm } \\
s\end{array}$ & $\begin{array}{c}\text { Au-ppm } \\
\text { aa }\end{array}$ & $\begin{array}{l}\text { AS-PPM } \\
\text { ICP }\end{array}$ \\
\hline $\begin{array}{l}\text { 9dh3467a } \\
\text { 9dh3467b } \\
\text { 9dh3468b } \\
\text { 9dh3468c } \\
\text { 9dh3469 }\end{array}$ & $\begin{array}{l}N \\
N \\
N \\
N \\
N\end{array}$ & $\begin{array}{r}N \\
7 \\
7 \\
<5 \\
5\end{array}$ & $\begin{array}{r}N \\
N \\
30 \\
N \\
N\end{array}$ & $\begin{array}{r}N \\
200 \\
300 \\
N \\
N\end{array}$ & $\begin{array}{l}<10 \\
150 \\
500 \\
100 \\
100\end{array}$ & $\begin{array}{l}N \\
N \\
N \\
N \\
N\end{array}$ & $\begin{array}{r}N \\
15 \\
150 \\
20 \\
10\end{array}$ & $\begin{array}{r}N \\
N \\
300 \\
N \\
N\end{array}$ & $\begin{array}{r}150 \\
150 \\
50 \\
300 \\
150\end{array}$ & $\begin{array}{l}N \\
N \\
N \\
N \\
N\end{array}$ & $\begin{array}{l}1.00 \\
.10 \\
<.05 \\
N \\
N\end{array}$ & $\begin{array}{r}29.0 \\
42.0 \\
300.0 \\
39.0 \\
5.0\end{array}$ \\
\hline $\begin{array}{l}\text { 9dh3470a } \\
\text { 9dh3470b } \\
9 d h 3470 c \\
\text { 9dh3479 } \\
9 d h 3472 a\end{array}$ & $\begin{array}{l}N \\
N \\
N \\
N \\
N\end{array}$ & $\begin{array}{c}N \\
N \\
N \\
N \\
15\end{array}$ & $\begin{array}{l}N \\
N \\
N \\
N \\
N\end{array}$ & $\begin{array}{r}1,000 \\
N \\
300 \\
N \\
N\end{array}$ & $\begin{array}{r}100 \\
10 \\
20 \\
10 \\
100\end{array}$ & $\begin{array}{l}N \\
N \\
N \\
N \\
N\end{array}$ & $\begin{array}{r}N \\
N \\
N \\
N \\
30\end{array}$ & $\begin{array}{l}N \\
N \\
N \\
N \\
N\end{array}$ & $\begin{array}{l}200 \\
150 \\
200 \\
150 \\
300\end{array}$ & $\begin{array}{l}\mathbf{N} \\
\mathbf{N} \\
\mathbf{N} \\
\mathbf{N} \\
\mathbf{N}\end{array}$ & $\begin{array}{l}3.20 \\
.55 \\
1.80 \\
.40 \\
\mathrm{~N}\end{array}$ & $\begin{array}{r}400.0 \\
1.8 \\
45.0 \\
44.0 \\
170.0\end{array}$ \\
\hline $\begin{array}{l}\text { 9dh3472b } \\
\text { 9dh3473a } \\
\text { 9dh3473b } \\
\text { 9dh3473c } \\
\text { 9dh3473d }\end{array}$ & $\begin{array}{l}N \\
N \\
N \\
N \\
N\end{array}$ & $\begin{array}{r}N \\
<5 \\
N \\
N \\
10\end{array}$ & $\begin{array}{l}N \\
N \\
N \\
N \\
N\end{array}$ & $\begin{array}{r}N \\
<100 \\
N \\
100 \\
500\end{array}$ & $\begin{array}{r}30 \\
100 \\
50 \\
50 \\
150\end{array}$ & $\begin{array}{l}N \\
N \\
N \\
N \\
N\end{array}$ & $\begin{array}{r}10 \\
<10 \\
N \\
N \\
15\end{array}$ & $\begin{array}{l}N \\
N \\
N \\
N \\
N\end{array}$ & $\begin{array}{l}100 \\
150 \\
100 \\
100 \\
200\end{array}$ & $\begin{array}{l}N \\
N \\
N \\
N \\
N\end{array}$ & $\begin{array}{r}<.05 \\
.55 \\
.80 \\
.20 \\
.15\end{array}$ & $\begin{array}{r}55.0 \\
230.0 \\
140.0 \\
6.7 \\
11.0\end{array}$ \\
\hline $\begin{array}{l}\text { 9dh3473e } \\
\text { 9dh3473f } \\
9 d h 3473 g \\
9 d h 3474 b \\
9 d h 3474 \mathrm{c}\end{array}$ & $\begin{array}{l}N \\
N \\
N \\
N \\
N\end{array}$ & $\begin{array}{r}N \\
N \\
N \\
5 \\
10\end{array}$ & $\begin{array}{r}N \\
N \\
N \\
20 \\
N\end{array}$ & $\begin{array}{r}N \\
<100 \\
N \\
300 \\
300\end{array}$ & $\begin{array}{r}10 \\
200 \\
10 \\
70 \\
70\end{array}$ & $\begin{array}{l}N \\
N \\
N \\
N \\
N\end{array}$ & $\begin{array}{r}N \\
<10 \\
N \\
50 \\
15\end{array}$ & $\begin{array}{r}N \\
N \\
N \\
300 \\
N\end{array}$ & $\begin{array}{r}30 \\
200 \\
150 \\
200 \\
150\end{array}$ & $\begin{array}{l}N \\
N \\
N \\
N \\
N\end{array}$ & $\begin{array}{l}.20 \\
2.00 \\
.25 \\
.05 \\
\mathrm{~N}\end{array}$ & $\begin{array}{r}9.8 \\
850.0 \\
30.0 \\
340.0 \\
16.0\end{array}$ \\
\hline $\begin{array}{l}\text { 9dh3476 } \\
\text { 9dh3477 } \\
\text { 9dh3478 } \\
\text { 9dh3479 } \\
\text { 9dh3480 }\end{array}$ & $\begin{array}{l}N \\
N \\
N \\
N \\
N\end{array}$ & $\begin{array}{r}5 \\
<5 \\
<5 \\
5 \\
7\end{array}$ & $\begin{array}{l}N \\
N \\
N \\
N \\
N\end{array}$ & $\begin{array}{r}100 \\
N \\
N \\
200 \\
N\end{array}$ & $\begin{array}{r}200 \\
300 \\
500 \\
500 \\
50\end{array}$ & $\begin{array}{l}\mathbf{N} \\
\mathbf{N} \\
\mathbf{N} \\
\mathbf{N} \\
\mathbf{N}\end{array}$ & $\begin{array}{r}<10 \\
20 \\
30 \\
50 \\
15\end{array}$ & $\begin{array}{r}N \\
N \\
N \\
300 \\
N\end{array}$ & $\begin{array}{r}100 \\
100 \\
200 \\
70 \\
100\end{array}$ & $\begin{array}{l}\mathrm{N} \\
\mathrm{N} \\
\mathrm{N} \\
\mathrm{N} \\
\mathrm{N}\end{array}$ & $\begin{array}{l}\mathrm{N} \\
\mathrm{N} \\
\mathrm{N} \\
\mathrm{N} \\
.10\end{array}$ & $\begin{array}{r}140.0 \\
84.0 \\
130.0 \\
520.0 \\
26.0\end{array}$ \\
\hline $\begin{array}{l}\text { 9dh3481 } \\
\text { 9dh3482 } \\
\text { 9dh3483 } \\
\text { 9dh3484 } \\
\text { 9dh3485 }\end{array}$ & $\begin{array}{l}N \\
N \\
N \\
N \\
N\end{array}$ & $\begin{array}{l}N \\
N \\
N \\
N \\
<5\end{array}$ & $\begin{array}{r}N \\
N \\
N \\
N \\
<10\end{array}$ & $\begin{array}{r}N \\
<100 \\
N \\
N \\
300\end{array}$ & $\begin{array}{r}10 \\
30 \\
<10 \\
50 \\
100\end{array}$ & $\begin{array}{l}N \\
N \\
N \\
N \\
N\end{array}$ & $\begin{array}{c}N \\
N \\
N \\
10 \\
50\end{array}$ & $\begin{array}{r}N \\
N \\
N \\
N \\
500\end{array}$ & $\begin{array}{r}150 \\
50 \\
70 \\
<10 \\
100\end{array}$ & $\begin{array}{l}\mathrm{N} \\
\mathrm{N} \\
\mathrm{N} \\
\mathrm{N} \\
\mathrm{N}\end{array}$ & $\begin{array}{l}.05 \\
\mathrm{~N}^{2} \\
.00 \\
.25 \\
.35\end{array}$ & $\begin{array}{r}30.0 \\
1.5 \\
15.0 \\
120.0 \\
570.0\end{array}$ \\
\hline $\begin{array}{l}\text { 9dh3486 } \\
\text { 9dh3487 } \\
\text { 9dh3488b } \\
\text { 9dh3488e } \\
\text { 9dh3488f }\end{array}$ & $\begin{array}{l}\mathbf{N} \\
\mathbf{N} \\
\mathbf{N} \\
\mathbf{N} \\
\mathbf{N}\end{array}$ & $\begin{array}{r}5 \\
<5 \\
N \\
5 \\
N\end{array}$ & $\begin{array}{r}N \\
N \\
N \\
50 \\
N\end{array}$ & $\begin{array}{r}200 \\
N \\
200 \\
300 \\
700\end{array}$ & $\begin{array}{r}30 \\
150 \\
30 \\
150 \\
50\end{array}$ & $\begin{array}{l}\mathbf{N} \\
\mathbf{N} \\
\mathbf{N} \\
\mathbf{N} \\
\mathbf{N}\end{array}$ & $\begin{array}{r}10 \\
N \\
N \\
N \\
N\end{array}$ & $\begin{array}{l}N \\
N \\
N \\
N \\
N\end{array}$ & $\begin{array}{r}500 \\
100 \\
70 \\
100 \\
50\end{array}$ & $\begin{array}{l}\mathrm{N} \\
\mathrm{N} \\
\mathrm{N} \\
\mathrm{N} \\
\mathrm{N}\end{array}$ & $\begin{array}{r}<.05 \\
.40 \\
.90 \\
16.80 \\
18.30\end{array}$ & $\begin{array}{l}70.0 \\
36.0 \\
68.0 \\
88.0 \\
11.0\end{array}$ \\
\hline $\begin{array}{l}\text { 9dh3488g } \\
9 \mathrm{dh} 3490 \\
9 \mathrm{dh} 3492 \\
9 \mathrm{dh} 3493 \mathrm{a} \\
9 \mathrm{dh} 3493 \mathrm{~b}\end{array}$ & $\begin{array}{l}N \\
N \\
N \\
N \\
N\end{array}$ & $\begin{array}{r}N \\
N \\
<5 \\
7 \\
10\end{array}$ & $\begin{array}{l}N \\
N \\
N \\
N \\
N\end{array}$ & $\begin{array}{r}200 \\
300 \\
300 \\
300 \\
\mathrm{~N}\end{array}$ & $\begin{array}{r}20 \\
70 \\
100 \\
100 \\
100\end{array}$ & $\begin{array}{l}\mathbf{N} \\
\mathbf{N} \\
\mathbf{N} \\
\mathbf{N} \\
\mathbf{N}\end{array}$ & $\begin{array}{r}N \\
N \\
<10 \\
15 \\
15\end{array}$ & $\begin{array}{l}N \\
N \\
N \\
N \\
N\end{array}$ & $\begin{array}{r}20 \\
150 \\
70 \\
150 \\
50\end{array}$ & $\begin{array}{l}N \\
N \\
N \\
N \\
N\end{array}$ & $\begin{array}{r}.80 \\
.80 \\
.20 \\
<.05 \\
.10\end{array}$ & $\begin{array}{c}15.0 \\
150.0 \\
76.0 \\
N \\
14.0\end{array}$ \\
\hline $\begin{array}{l}9 \mathrm{dh} 3493 \mathrm{c} \\
9 \mathrm{dh} 3493 \mathrm{~d} \\
9 \mathrm{dh} 3493 \mathrm{e} \\
9 \mathrm{dh} 3493 \mathrm{f} \\
9 \mathrm{dh} 3493 \mathrm{~g}\end{array}$ & $\begin{array}{l}N \\
N \\
N \\
N \\
N\end{array}$ & $\begin{array}{l}N \\
N \\
N \\
N \\
N\end{array}$ & $\begin{array}{l}N \\
N \\
N \\
N \\
N\end{array}$ & $\begin{array}{r}N \\
N \\
N \\
N \\
<100\end{array}$ & $\begin{array}{l}10 \\
10 \\
10 \\
10 \\
15\end{array}$ & $\begin{array}{l}N \\
N \\
N \\
N \\
N\end{array}$ & $\begin{array}{l}N \\
N \\
N \\
N \\
N\end{array}$ & $\begin{array}{l}N \\
N \\
N \\
N \\
N\end{array}$ & $\begin{array}{r}20 \\
70 \\
30 \\
100 \\
20\end{array}$ & $\begin{array}{l}N \\
N \\
N \\
N \\
N\end{array}$ & $\begin{array}{r}.10 \\
.45 \\
.65 \\
2.65 \\
.55\end{array}$ & $\begin{array}{r}9.4 \\
6.5 \\
1.5 \\
9.6 \\
35.0\end{array}$ \\
\hline
\end{tabular}


TABLE 4--RESULTS OF ANALYSES, DETROIT MINING DISTRICT, UTAH--Continued

\begin{tabular}{|c|c|c|c|c|c|c|c|c|c|c|}
\hline Sample & $\begin{array}{l}\text { BI-PPM } \\
\text { ICP }\end{array}$ & $\begin{array}{l}\text { CD-PPM } \\
\text { ICP }\end{array}$ & $\begin{array}{l}\text { SB-PPM } \\
\text { ICP }\end{array}$ & $\begin{array}{l}\text { ZN-PPM } \\
\text { ICP }\end{array}$ & $\begin{array}{c}\text { Na-pct. } \\
s\end{array}$ & $\underset{s}{\text { P-pct. }}$ & $\begin{array}{l}\text { Ga-ppm } \\
\mathbf{s}\end{array}$ & $\begin{array}{l}\text { Ge-ppm } \\
\text { s }\end{array}$ & $\begin{array}{l}\text { HG-PPM } \\
\mathrm{CV}\end{array}$ & $\begin{array}{l}\text { F\% } \\
\text { ISE }\end{array}$ \\
\hline $\begin{array}{l}\text { 9dh3467a } \\
\text { 9dh3467b } \\
9 \mathrm{dh} 3468 \mathrm{~b} \\
\text { 9dh3468c } \\
\text { 9dh3469 }\end{array}$ & $\begin{array}{c}.62 \\
N^{N .00} \\
N \\
N\end{array}$ & $\begin{array}{l}\mathrm{N} \\
\mathrm{N} \\
.170 \\
\mathrm{~N} \\
.081\end{array}$ & $\begin{array}{l}3.50 \\
2.10 \\
9.40 \\
1.60 \\
\mathrm{~N}\end{array}$ & $\begin{array}{r}1.70 \\
2.00 \\
170.00 \\
64.00 \\
25.00\end{array}$ & $\begin{array}{l}N \\
<.2 \\
3.0 \\
.5 \\
N\end{array}$ & $\begin{array}{l}N \\
<.2 \\
N \\
<.2 \\
<.2\end{array}$ & $\begin{array}{r}N \\
10 \\
15 \\
15 \\
15\end{array}$ & $\begin{array}{l}\mathrm{N} \\
\mathrm{N} \\
\mathrm{N} \\
\mathrm{N} \\
\mathrm{N}\end{array}$ & $\begin{array}{l}.40 \\
.10 \\
.14 \\
.02 \\
\mathrm{~N}\end{array}$ & $\begin{array}{l}<.01 \\
.06 \\
.03 \\
.07 \\
.06\end{array}$ \\
\hline $\begin{array}{l}\text { 9dh3470a } \\
9 \mathrm{dh} 3470 \mathrm{~b} \\
9 \mathrm{dh} 3470 \mathrm{c} \\
9 \mathrm{dh} 3471 \\
\text { 9dh3472a }\end{array}$ & $\begin{array}{l}23.00 \\
N \\
2.20 \\
2.90 \\
N\end{array}$ & $\begin{array}{l}.063 \\
N \\
N \\
.037 \\
.087\end{array}$ & $\begin{array}{r}34.00 \\
1.50 \\
5.30 \\
5.10 \\
11.00\end{array}$ & $\begin{array}{c}N \\
.32 \\
.50 \\
.10 \\
49.00\end{array}$ & $\begin{array}{l}N \\
N \\
N \\
N \\
N\end{array}$ & $\begin{array}{l}.2 \\
N \\
N \\
N \\
N\end{array}$ & $\begin{array}{r}30 \\
N \\
5 \\
<5 \\
20\end{array}$ & $\begin{array}{l}\text { N } \\
N \\
N \\
N \\
N\end{array}$ & $\begin{array}{r}.24 \\
.04 \\
1.40 \\
.44 \\
.02\end{array}$ & $\begin{array}{r}.06 \\
.01 \\
<.01 \\
<.01 \\
.05\end{array}$ \\
\hline $\begin{array}{l}\text { 9dh3472b } \\
\text { 9dh3473a } \\
\text { 9dh3473b } \\
\text { 9dh3473c } \\
\text { 9dh3473d }\end{array}$ & $\begin{array}{c}N \\
10.00 \\
2.60 \\
.65 \\
N\end{array}$ & $\begin{array}{l}.110 \\
.085 \\
.040 \\
N \\
N\end{array}$ & $\begin{array}{r}2.20 \\
23.00 \\
5.70 \\
.68 \\
.65\end{array}$ & $\begin{array}{r}47.00 \\
55.00 \\
9.90 \\
1.40 \\
1.20\end{array}$ & $\begin{array}{l}\text { N } \\
\text { N } \\
\text { N } \\
N \\
N\end{array}$ & $\begin{array}{l}\mathrm{N} \\
\mathrm{N} \\
\mathrm{N} \\
<.2 \\
.2\end{array}$ & $\begin{array}{r}10 \\
7 \\
<5 \\
<5 \\
10\end{array}$ & $\begin{array}{l}\mathrm{N} \\
\mathrm{N} \\
\mathrm{N} \\
\mathrm{N} \\
\mathrm{N}\end{array}$ & $\begin{array}{l}.04 \\
.04 \\
.12 \\
.02 \\
.02\end{array}$ & $\begin{array}{r}.03 \\
.04 \\
<.01 \\
.01 \\
.05\end{array}$ \\
\hline $\begin{array}{l}\text { 9dh3473e } \\
\text { 9dh3473f } \\
\text { 9dh3473g } \\
9 \mathrm{dh} 3474 \mathrm{~b} \\
\text { 9dh3474c }\end{array}$ & $\begin{array}{c}1.00 \\
11.00 \\
N \\
.61 \\
.73\end{array}$ & $\begin{array}{l}N \\
.100 \\
N \\
2.600 \\
.037\end{array}$ & $\begin{array}{r}.87 \\
35.00 \\
2.40 \\
12.00 \\
1.30\end{array}$ & $\begin{array}{r}.99 \\
50.00 \\
3.30 \\
190.00 \\
18.00\end{array}$ & $\begin{array}{l}N \\
N \\
N \\
N \\
N\end{array}$ & $\begin{array}{l}<.2 \\
N \\
<.2 \\
N \\
.2\end{array}$ & $\begin{array}{r}N \\
10 \\
N \\
10 \\
15\end{array}$ & $\begin{array}{l}N \\
N \\
N \\
N \\
N\end{array}$ & $\begin{array}{r}.02 \\
.20 \\
.10 \\
2.10 \\
.04\end{array}$ & $\begin{array}{l}<.01 \\
<.01 \\
<.01 \\
.04 \\
.06\end{array}$ \\
\hline $\begin{array}{l}\text { 9dh3476 } \\
\text { 9dh3477 } \\
\text { 9dh3478 } \\
\text { 9dh3479 } \\
\text { 9dh3480 }\end{array}$ & $\begin{array}{l}.86 \\
1.20 \\
N \\
N \\
.88\end{array}$ & $\begin{array}{l}.082 \\
.340 \\
.720 \\
.270 \\
N^{2}\end{array}$ & $\begin{array}{r}13.00 \\
6.90 \\
6.60 \\
52.00 \\
2.10\end{array}$ & $\begin{array}{c}99.00 \\
61.00 \\
120.00 \\
95.00 \\
\mathrm{~N}\end{array}$ & $\begin{array}{l}1.0 \\
N \\
.2 \\
1.5 \\
N\end{array}$ & $\begin{array}{l}<.2 \\
N \\
N \\
N \\
N\end{array}$ & $\begin{array}{l}15 \\
15 \\
10 \\
15 \\
20\end{array}$ & $\begin{array}{l}\mathrm{N} \\
\mathrm{N} \\
\mathrm{N} \\
\mathrm{N} \\
\mathrm{N}\end{array}$ & $\begin{array}{l}.02 \\
.08 \\
.06 \\
N \\
.26\end{array}$ & $\begin{array}{l}.06 \\
.01 \\
.04 \\
.02 \\
.08\end{array}$ \\
\hline $\begin{array}{l}\text { 9dh3481 } \\
\text { 9dh3482 } \\
\text { 9dh3483 } \\
\text { 9dh3484 } \\
\text { 9dh3485 }\end{array}$ & $\begin{array}{l}.70 \\
N \\
.74 \\
3.90 \\
8.70\end{array}$ & $\begin{array}{l}.065 \\
N \\
N \\
.720 \\
2.900\end{array}$ & $\begin{array}{l}2.00 \\
N \\
2.00 \\
4.10 \\
26.00\end{array}$ & $\begin{array}{r}N \\
22.00 \\
28.00 \\
110.00 \\
310.00\end{array}$ & $\begin{array}{c}N \\
N \\
N \\
N \\
1.0\end{array}$ & $\begin{array}{l}<.2 \\
<.2 \\
<.2 \\
N \\
N\end{array}$ & $\begin{array}{r}N \\
<5 \\
N \\
<5 \\
10\end{array}$ & $\begin{array}{l}N \\
N \\
N \\
N \\
N\end{array}$ & $\begin{array}{l}.26 \\
N \\
.30 \\
.24 \\
.60\end{array}$ & $\begin{array}{r}<.01 \\
.01 \\
<.01 \\
.01 \\
.04\end{array}$ \\
\hline $\begin{array}{l}\text { 9dh3486 } \\
\text { 9dh3487 } \\
\text { 9dh3488b } \\
\text { 9dh3488e } \\
\text { 9dh3488f }\end{array}$ & $\begin{array}{l}3.30 \\
N \\
1.90 \\
6.70 \\
.64\end{array}$ & $\begin{array}{l}N \\
.031 \\
N \\
N \\
N\end{array}$ & $\begin{array}{r}3.40 \\
3.30 \\
31.00 \\
3.70 \\
1.10\end{array}$ & $\begin{array}{r}26.00 \\
11.00 \\
2.10 \\
2.80 \\
.65\end{array}$ & $\begin{array}{l}\text { N } \\
.7 \\
N \\
N \\
N\end{array}$ & $\begin{array}{l}<.2 \\
N \\
N \\
<.2 \\
.2\end{array}$ & $\begin{array}{r}10 \\
7 \\
<5 \\
30 \\
5\end{array}$ & $\begin{array}{l}\text { N } \\
\text { N } \\
\text { N } \\
\text { N } \\
\text { N }\end{array}$ & $\begin{array}{l}.18 \\
i^{102} \\
.02 \\
\mathrm{~N}^{20}\end{array}$ & $\begin{array}{l}.04 \\
.04 \\
.01 \\
.14 \\
.03\end{array}$ \\
\hline $\begin{array}{l}\text { 9dh3488g } \\
\text { 9dh3490 } \\
\text { 9dh3492 } \\
\text { 9dh3493a } \\
\text { 9dh3493b }\end{array}$ & $\begin{array}{l}N \\
.89 \\
3.20 \\
N \\
.63\end{array}$ & $\begin{array}{l}\mathrm{N} \\
\mathrm{N} \\
.068 \\
\mathrm{~N} \\
.091\end{array}$ & $\begin{array}{l}3.10 \\
9.00 \\
4.20 \\
N \\
1.10\end{array}$ & $\begin{array}{r}.32 \\
12.00 \\
13.00 \\
2.20 \\
14.00\end{array}$ & $\begin{array}{c}<.2 \\
N \\
<.2 \\
.7 \\
1.5\end{array}$ & $\begin{array}{l}<.2 \\
N \\
N \\
.2 \\
<.2\end{array}$ & $\begin{array}{r}20 \\
5 \\
15 \\
10 \\
30\end{array}$ & $\begin{array}{l}\text { N } \\
N \\
N \\
N \\
N\end{array}$ & $\begin{array}{l}\mathrm{N} \\
\mathrm{N} \\
.10 \\
.20 \\
1.30\end{array}$ & $\begin{array}{l}.03 \\
.04 \\
.05 \\
.05 \\
.06\end{array}$ \\
\hline $\begin{array}{l}\text { 9dh3493c } \\
\text { 9dh3493d } \\
9 \mathrm{dh} 3493 \mathrm{e} \\
9 \mathrm{dh} 3493 \mathrm{f} \\
9 \mathrm{dh} 3493 \mathrm{~g}\end{array}$ & $\begin{array}{l}N \\
1.10 \\
N \\
1.30 \\
1.10\end{array}$ & $\begin{array}{l}N \\
N \\
N \\
N \\
N\end{array}$ & $\begin{array}{l}N \\
N \\
N \\
6.20 \\
7.20\end{array}$ & $\begin{array}{r}47.00 \\
21.00 \\
.16 \\
1.10 \\
2.90\end{array}$ & $\begin{array}{l}\text { N } \\
N \\
N \\
N \\
N\end{array}$ & $\begin{array}{l}<.2 \\
<.2 \\
<.2 \\
<.2 \\
N\end{array}$ & $\begin{array}{l}<5 \\
N \\
N \\
N \\
5\end{array}$ & $\begin{array}{l}N \\
N \\
N \\
N \\
N\end{array}$ & $\begin{array}{c}10.40 \\
1.60 \\
N \\
.44 \\
.12\end{array}$ & $\begin{array}{l}<.01 \\
<.01 \\
<.01 \\
<.01 \\
.02\end{array}$ \\
\hline
\end{tabular}


TABLE 4--RESULTS OF ANALYSES, DETROIT MINING DISTRICT, UTAH--Continued

\begin{tabular}{|c|c|c|c|c|c|c|c|c|c|c|}
\hline Sample & Latitude & Long i tude & $\begin{array}{c}\text { Fe-pct } \\
\text { s }\end{array}$ & $\begin{array}{c}\text { Mg-pct } \\
\text { s }\end{array}$ & $\begin{array}{c}\mathrm{Ca}-\mathrm{pct} \\
\mathrm{s}\end{array}$ & $\begin{array}{c}\text { Ti-pct. } \\
\mathbf{s}\end{array}$ & $\begin{array}{l}\text { Mn-ppm } \\
s\end{array}$ & $\begin{array}{l}\text { Ag-ppm } \\
\mathrm{s}\end{array}$ & $\begin{array}{l}\text { As-ppm } \\
\text { s }\end{array}$ & $\begin{array}{c}\text { Au-ppm } \\
\text { s }\end{array}$ \\
\hline $\begin{array}{l}9 \mathrm{dh} 3493 \mathrm{~h} \\
80 \mathrm{H} 3500 \\
8013501 \\
8013502 \\
8013503\end{array}$ & $\begin{array}{lll}39 & 31 & 39 \\
39 & 34 & 33 \\
39 & 34 & 30 \\
39 & 34 & 28 \\
39 & 33 & 57\end{array}$ & $\begin{array}{rrr}113 & 0 & 25 \\
113 & 0 & 7 \\
112 & 59 & 57 \\
112 & 59 & 49 \\
112 & 59 & 43\end{array}$ & $\begin{array}{r}7.00 \\
.05 \\
2.00 \\
2.00 \\
5.00\end{array}$ & $\begin{array}{l}<.02 \\
<.02 \\
.05 \\
.02 \\
<.02\end{array}$ & $\begin{array}{r}.05 \\
.05 \\
.20 \\
.20 \\
<.05\end{array}$ & $\begin{array}{l}.150 \\
.020 \\
.300 \\
.150 \\
.050\end{array}$ & $\begin{array}{l}50 \\
20 \\
30 \\
20 \\
15\end{array}$ & $\begin{array}{l}N \\
N \\
N \\
N \\
N\end{array}$ & $\begin{array}{r}N \\
N \\
<200 \\
N \\
N\end{array}$ & $\begin{array}{l}N \\
N \\
N \\
N \\
N\end{array}$ \\
\hline $\begin{array}{l}8013504 \\
80 H 3505 \\
80 H 3506 \\
80 H 3507 \\
80 H 3508\end{array}$ & $\begin{array}{lll}39 & 34 & 22 \\
39 & 34 & 24 \\
39 & 33 & 56 \\
39 & 34 & 16 \\
39 & 34 & 35\end{array}$ & $\begin{array}{rrr}112 & 59 & 44 \\
113 & 0 & 14 \\
113 & 0 & 28 \\
113 & 1 & 23 \\
113 & 1 & 5\end{array}$ & $\begin{array}{l}1.50 \\
5.00 \\
3.00 \\
7.00 \\
1.00\end{array}$ & $\begin{array}{l}<.02 \\
.02 \\
.03 \\
.02 \\
.05\end{array}$ & $\begin{array}{l}.05 \\
.07 \\
.10 \\
.05 \\
.50\end{array}$ & $\begin{array}{l}.150 \\
.100 \\
.050 \\
.020 \\
.020\end{array}$ & $\begin{array}{l}20 \\
10 \\
30 \\
15 \\
50\end{array}$ & $\begin{array}{l}N \\
N \\
N \\
N \\
N\end{array}$ & $\begin{array}{l}N \\
N \\
N \\
N \\
N\end{array}$ & $\begin{array}{l}N \\
N \\
N \\
N \\
N\end{array}$ \\
\hline $\begin{array}{l}80 H 3510 \\
80 H 3511 \\
80 H 3512 \\
80 H 3513 \\
80 H 3514\end{array}$ & $\begin{array}{lll}39 & 33 & 53 \\
39 & 33 & 47 \\
39 & 33 & 47 \\
39 & 33 & 44 \\
39 & 33 & 42\end{array}$ & $\begin{array}{lll}113 & 0 & 51 \\
113 & 0 & 45 \\
113 & 0 & 45 \\
113 & 1 & 12 \\
113 & 1 & 26\end{array}$ & $\begin{array}{r}1.50 \\
1.50 \\
10.00 \\
10.00 \\
15.00\end{array}$ & $\begin{array}{l}<.02 \\
.03 \\
.03 \\
.03 \\
.70\end{array}$ & $\begin{array}{l}.07 \\
.15 \\
.10 \\
.15 \\
.20\end{array}$ & $\begin{array}{l}.100 \\
.300 \\
.150 \\
.150 \\
.100\end{array}$ & $\begin{array}{r}15 \\
15 \\
10 \\
100 \\
>5,000\end{array}$ & $\begin{array}{l}N \\
N \\
N \\
N \\
N\end{array}$ & $\begin{array}{r}N \\
N \\
N \\
200 \\
5,000\end{array}$ & $\begin{array}{l}N \\
N \\
N \\
N \\
N\end{array}$ \\
\hline $\begin{array}{l}80 H 3515 \\
80 H 3516 \\
80 H 3517 \\
8013518 \\
80 H 3519\end{array}$ & $\begin{array}{lll}39 & 33 & 46 \\
39 & 33 & 53 \\
39 & 33 & 57 \\
39 & 33 & 40 \\
39 & 33 & 46\end{array}$ & $\begin{array}{rrr}113 & 0 & 25 \\
113 & 0 & 18 \\
113 & 0 & 10 \\
112 & 59 & 54 \\
113 & 0 & 4\end{array}$ & $\begin{array}{l}3.00 \\
3.00 \\
3.00 \\
5.00 \\
7.00\end{array}$ & $\begin{array}{l}.02 \\
.05 \\
.02 \\
.02 \\
.20\end{array}$ & $\begin{array}{l}<.05 \\
.15 \\
.30 \\
.10 \\
.15\end{array}$ & $\begin{array}{l}.070 \\
.200 \\
.150 \\
.100 \\
.300\end{array}$ & $\begin{array}{r}70 \\
20 \\
15 \\
100 \\
5,000\end{array}$ & $\begin{array}{l}N \\
5.00 \\
N \\
N\end{array}$ & $\begin{array}{l}N \\
N \\
N \\
N \\
N\end{array}$ & $\begin{array}{l}N \\
N \\
N \\
N \\
N\end{array}$ \\
\hline $\begin{array}{l}80 H 3520 \\
80 H 3521 \\
80 H 3522 \\
80 H 3523 \\
80 H 3524\end{array}$ & $\begin{array}{lll}39 & 33 & 26 \\
39 & 33 & 18 \\
39 & 33 & 22 \\
39 & 33 & 25 \\
39 & 33 & 25\end{array}$ & $\begin{array}{rrr}113 & 0 & 44 \\
113 & 0 & 52 \\
113 & 0 & 43 \\
113 & 0 & 37 \\
113 & 0 & 8\end{array}$ & $\begin{array}{l}3.00 \\
2.00 \\
.10 \\
3.00 \\
5.00\end{array}$ & $\begin{array}{l}.10 \\
.15 \\
.03 \\
.10 \\
.10\end{array}$ & $\begin{array}{l}.20 \\
.50 \\
.20 \\
.30 \\
.30\end{array}$ & $\begin{array}{r}.200 \\
.200 \\
1.000 \\
.300 \\
.300\end{array}$ & $\begin{array}{l}50 \\
50 \\
70 \\
30 \\
70\end{array}$ & $\begin{array}{l}N \\
N \\
2.00 \\
2.00 \\
.50\end{array}$ & $\begin{array}{r}N \\
N \\
N \\
N \\
300\end{array}$ & $\begin{array}{l}N \\
N \\
N \\
N \\
N\end{array}$ \\
\hline $\begin{array}{l}80 H 3526 \\
8013527 \\
80 H 3528 \\
80 H 3529 \\
80 H 3530\end{array}$ & $\begin{array}{rrr}39 & 33 & 3 \\
39 & 33 & 38 \\
39 & 33 & 18 \\
39 & 33 & 9 \\
39 & 33 & 1\end{array}$ & $\begin{array}{rrrr}113 & 0 & 4 \\
112 & 59 & 53 \\
113 & 0 & 27 \\
113 & 0 & 41 \\
113 & 0 & 24\end{array}$ & $\begin{array}{r}.50 \\
10.00 \\
2.00 \\
1.50 \\
5.00\end{array}$ & $\begin{array}{l}.02 \\
.10 \\
.03 \\
.05 \\
.05\end{array}$ & $\begin{array}{r}.10 \\
1.00 \\
.20 \\
.30 \\
.20\end{array}$ & $\begin{array}{l}.500 \\
.200 \\
.200 \\
.300 \\
.300\end{array}$ & $\begin{array}{r}70 \\
500 \\
50 \\
20 \\
70\end{array}$ & $\begin{array}{l}\dot{N}^{.70} \\
10.00 \\
<.50\end{array}$ & $\begin{array}{r}N \\
500 \\
N \\
N \\
N\end{array}$ & $\begin{array}{l}N \\
N \\
N \\
N \\
N\end{array}$ \\
\hline $\begin{array}{l}80 h 3532 \\
80 h 3533 \\
80 h 3534 \\
80 h 3535 \\
80 h 3536\end{array}$ & $\begin{array}{rrr}39 & 33 & 1 \\
39 & 33 & 4 \\
39 & 33 & 4 \\
39 & 32 & 46 \\
39 & 32 & 51\end{array}$ & $\begin{array}{lll}113 & 0 & 25 \\
113 & 0 & 10 \\
113 & 0 & 10 \\
113 & 0 & 29 \\
113 & 0 & 18\end{array}$ & $\begin{array}{l}1.00 \\
2.00 \\
1.50 \\
2.00 \\
1.50\end{array}$ & $\begin{array}{l}.03 \\
.07 \\
.10 \\
.03 \\
.03\end{array}$ & $\begin{array}{l}.50 \\
.50 \\
.70 \\
.30 \\
.50\end{array}$ & $\begin{array}{l}.300 \\
.500 \\
.200 \\
.200 \\
.150\end{array}$ & $\begin{array}{l}70 \\
70 \\
50 \\
10 \\
30\end{array}$ & $\begin{array}{l}5.00 \\
N \\
N \\
N \\
N\end{array}$ & $\begin{array}{l}N \\
N \\
N \\
N \\
N\end{array}$ & $\begin{array}{l}N \\
N \\
N \\
N \\
N\end{array}$ \\
\hline $\begin{array}{l}8013537 \\
8013538 \\
8013539 \\
8013540 \\
8013541\end{array}$ & $\begin{array}{lll}39 & 32 & 51 \\
39 & 32 & 57 \\
39 & 32 & 48 \\
39 & 32 & 38 \\
39 & 32 & 29\end{array}$ & $\begin{array}{rrr}112 & 59 & 55 \\
112 & 59 & 53 \\
112 & 59 & 40 \\
112 & 59 & 32 \\
112 & 59 & 1\end{array}$ & $\begin{array}{l}.70 \\
3.00 \\
5.00 \\
2.00 \\
1.50\end{array}$ & $\begin{array}{r}.02 \\
.02 \\
.02 \\
.02 \\
<.02\end{array}$ & $\begin{array}{l}.20 \\
.15 \\
.20 \\
.50 \\
.05\end{array}$ & $\begin{array}{l}.150 \\
.300 \\
.200 \\
.200 \\
.150\end{array}$ & $\begin{array}{r}50 \\
70 \\
20 \\
150 \\
10\end{array}$ & $\begin{array}{l}2_{2.00}^{N} \\
.50 \\
\mathbb{N}_{N}\end{array}$ & $\begin{array}{r}N \\
300 \\
N \\
N \\
N\end{array}$ & $\begin{array}{l}N \\
N \\
N \\
N \\
N\end{array}$ \\
\hline $\begin{array}{l}8013542 \\
8013543 \\
8013544 \\
8013545 \\
8013546\end{array}$ & $\begin{array}{lll}39 & 32 & 41 \\
39 & 32 & 49 \\
39 & 32 & 56 \\
39 & 32 & 31 \\
39 & 32 & 34\end{array}$ & $\begin{array}{rrr}112 & 59 & 4 \\
112 & 58 & 42 \\
112 & 58 & 53 \\
112 & 59 & 47 \\
112 & 59 & 48\end{array}$ & $\begin{array}{r}2.00 \\
3.00 \\
10.00 \\
5.00 \\
1.00\end{array}$ & $\begin{array}{l}.05 \\
.20 \\
.20 \\
.10 \\
.03\end{array}$ & $\begin{array}{l}.50 \\
5.00 \\
2.00 \\
.20 \\
.10\end{array}$ & $\begin{array}{l}.500 \\
.070 \\
.100 \\
.500 \\
.150\end{array}$ & $\begin{array}{r}150 \\
50 \\
100 \\
20 \\
20\end{array}$ & $\begin{array}{l}1.00 \\
2.00 \\
N \\
N \\
N\end{array}$ & $\begin{array}{r}N \\
N \\
200 \\
N \\
N\end{array}$ & $\begin{array}{l}N \\
N \\
N \\
N \\
N\end{array}$ \\
\hline
\end{tabular}


TABLE 4--RESULTS OF ANALYSES, DETROIT MINING DISTRICT, UTAH--Continued

\begin{tabular}{|c|c|c|c|c|c|c|c|c|c|c|c|c|c|}
\hline Sample & $\begin{array}{c}\text { B-ppm } \\
\text { s }\end{array}$ & $\begin{array}{c}\text { Ba-ppm } \\
s\end{array}$ & $\begin{array}{c}\text { Be-ppm } \\
\mathbf{s}\end{array}$ & $\begin{array}{c}B i-p p m \\
s\end{array}$ & $\begin{array}{c}\text { Cd-ppm } \\
s\end{array}$ & $\begin{array}{c}\text { Co-ppm } \\
s\end{array}$ & $\begin{array}{c}\mathrm{Cr}-\mathrm{ppm} \\
\mathrm{s}\end{array}$ & $\begin{array}{c}\text { Cu-ppm } \\
s\end{array}$ & $\begin{array}{l}\text { La-ppm } \\
\quad s\end{array}$ & $\begin{array}{c}\text { Mo-ppm } \\
\mathrm{s}\end{array}$ & $\begin{array}{c}\text { Nb-ppm } \\
s\end{array}$ & $\begin{array}{c}N i-p p m \\
s\end{array}$ & $\begin{array}{c}\text { Pb-ppm } \\
\text { s }\end{array}$ \\
\hline $\begin{array}{l}9 d h 3493 h \\
80 h 3500 \\
8013501 \\
8013502 \\
8013503\end{array}$ & $\begin{array}{r}<10 \\
10 \\
<10 \\
<10 \\
<10\end{array}$ & $\begin{array}{r}100 \\
30 \\
50 \\
200 \\
200\end{array}$ & $\begin{array}{c}<1.0 \\
N \\
N \\
N \\
N\end{array}$ & $\begin{array}{l}N \\
N \\
N \\
N \\
N\end{array}$ & $\begin{array}{l}N \\
N \\
N \\
N \\
N\end{array}$ & $\begin{array}{c}N \\
N \\
15 \\
N \\
N\end{array}$ & $\begin{array}{r}20 \\
N \\
50 \\
N \\
N\end{array}$ & $\begin{array}{r}10 \\
5 \\
30 \\
5 \\
5\end{array}$ & $\begin{array}{r}<50 \\
N \\
<50 \\
N \\
N\end{array}$ & $\begin{array}{l}N \\
N \\
N \\
N \\
N\end{array}$ & $\begin{array}{l}N \\
N \\
N \\
N \\
N\end{array}$ & $\begin{array}{r}N \\
<5 \\
15 \\
N \\
N\end{array}$ & $\begin{array}{r}50 \\
N \\
10 \\
<10 \\
<10\end{array}$ \\
\hline $\begin{array}{l}8013504 \\
80 H 3505 \\
80 H 3506 \\
80 H 3507 \\
80 H 3508\end{array}$ & $\begin{array}{r}10 \\
<10 \\
<10 \\
N \\
<10\end{array}$ & $\begin{array}{r}200 \\
30 \\
300 \\
200 \\
200\end{array}$ & $\begin{array}{l}\mathbf{N} \\
\mathbf{N} \\
\mathbf{N} \\
\mathbf{N} \\
\mathbf{N}\end{array}$ & $\begin{array}{l}N \\
N \\
N \\
N \\
N\end{array}$ & $\begin{array}{l}\mathbf{N} \\
\mathbf{N} \\
\mathbf{N} \\
\mathbf{N} \\
\mathbf{N}\end{array}$ & $\begin{array}{r}N \\
<10 \\
N \\
N \\
N\end{array}$ & $\begin{array}{r}<10 \\
<10 \\
N \\
N \\
N\end{array}$ & $\begin{array}{r}7 \\
20 \\
5 \\
30 \\
7\end{array}$ & $\begin{array}{l}N \\
N \\
N \\
N \\
N\end{array}$ & $\begin{array}{l}N \\
N \\
N \\
N \\
N\end{array}$ & $\begin{array}{l}N \\
N \\
N \\
N \\
N\end{array}$ & $\begin{array}{l}<5 \\
<5 \\
<5 \\
<5 \\
<5\end{array}$ & $\begin{array}{r}<10 \\
<10 \\
N \\
N \\
<10\end{array}$ \\
\hline $\begin{array}{l}80 H 3510 \\
80 H 3511 \\
80 H 3512 \\
80 H 3513 \\
80 H 3514\end{array}$ & $\begin{array}{r}<10 \\
<10 \\
N \\
N \\
<10\end{array}$ & $\begin{array}{r}100 \\
200 \\
500 \\
300 \\
1,000\end{array}$ & $\begin{array}{l}N \\
N \\
N \\
N \\
3.0\end{array}$ & $\begin{array}{l}N \\
N \\
N \\
N \\
N\end{array}$ & $\begin{array}{l}N \\
N \\
N \\
N \\
N\end{array}$ & $\begin{array}{r}N \\
N \\
N \\
<10 \\
10\end{array}$ & $\begin{array}{r}N \\
<10 \\
10 \\
10 \\
<10\end{array}$ & $\begin{array}{r}5 \\
50 \\
50 \\
10 \\
10\end{array}$ & $\begin{array}{r}N \\
N \\
N \\
<50 \\
N\end{array}$ & $\begin{array}{l}N \\
N \\
5 \\
N \\
N\end{array}$ & $\begin{array}{l}N \\
N \\
N \\
N \\
N\end{array}$ & $\begin{array}{r}<5 \\
N \\
<5 \\
10 \\
7\end{array}$ & $\begin{array}{r}<10 \\
30 \\
10 \\
100 \\
<10\end{array}$ \\
\hline $\begin{array}{l}80 H 3515 \\
80 H 3516 \\
80 H 3517 \\
8013518 \\
80 H 3519\end{array}$ & $\begin{array}{l}10 \\
10 \\
10 \\
10 \\
20\end{array}$ & $\begin{array}{l}150 \\
500 \\
100 \\
100 \\
700\end{array}$ & $\begin{array}{l}N \\
N \\
N \\
1.5 \\
1.0\end{array}$ & $\begin{array}{l}N \\
N \\
N \\
N \\
N\end{array}$ & $\begin{array}{l}N \\
N \\
N \\
N \\
N\end{array}$ & $\begin{array}{l}N \\
N \\
N \\
N \\
50\end{array}$ & $\begin{array}{r}<10 \\
N \\
N \\
N \\
<10\end{array}$ & $\begin{array}{r}<5 \\
<5 \\
7 \\
7 \\
50\end{array}$ & $\begin{array}{r}N \\
<50 \\
N \\
N \\
N\end{array}$ & $\begin{array}{l}N \\
5 \\
N \\
N \\
5\end{array}$ & $\begin{array}{l}N \\
N \\
N \\
N \\
N\end{array}$ & $\begin{array}{l}<5 \\
<5 \\
<5 \\
<5 \\
30\end{array}$ & $\begin{array}{r}<10 \\
<10 \\
N \\
30 \\
30\end{array}$ \\
\hline $\begin{array}{l}80 H 3520 \\
80 H 3521 \\
80 H 3522 \\
80 H 3523 \\
80 H 3524\end{array}$ & $\begin{array}{l}15 \\
10 \\
15 \\
15 \\
15\end{array}$ & $\begin{array}{l}700 \\
300 \\
150 \\
300 \\
300\end{array}$ & $\begin{array}{c}N \\
N \\
<1.0 \\
1.0 \\
1.5\end{array}$ & $\begin{array}{l}N \\
N \\
N \\
N \\
N\end{array}$ & $\begin{array}{l}N \\
N \\
N \\
N \\
N\end{array}$ & $\begin{array}{r}N \\
N \\
N \\
20 \\
<10\end{array}$ & $\begin{array}{r}20 \\
15 \\
30 \\
30 \\
N\end{array}$ & $\begin{array}{r}10 \\
50 \\
5 \\
300 \\
50\end{array}$ & $\begin{array}{r}<50 \\
50 \\
<50 \\
70 \\
50\end{array}$ & $\begin{array}{l}20 \\
20 \\
15 \\
10 \\
<5\end{array}$ & $\begin{array}{r}N \\
N \\
20 \\
N \\
N\end{array}$ & $\begin{array}{r}5 \\
7 \\
<5 \\
30 \\
10\end{array}$ & $\begin{array}{r}200 \\
300 \\
30 \\
200 \\
500\end{array}$ \\
\hline $\begin{array}{l}80 H 3526 \\
8013527 \\
80 H 3528 \\
80 H 3529 \\
80 H 3530\end{array}$ & $\begin{array}{l}10 \\
20 \\
15 \\
20 \\
15\end{array}$ & $\begin{array}{r}200 \\
200 \\
500 \\
1,500 \\
100\end{array}$ & $\begin{array}{r}<1.0 \\
5.0 \\
<1.0 \\
<1.0 \\
1.5\end{array}$ & $\begin{array}{l}N \\
N \\
N \\
N \\
N\end{array}$ & $\begin{array}{l}N \\
N \\
N \\
N \\
N\end{array}$ & $\begin{array}{r}N \\
<10 \\
30 \\
<10 \\
<10\end{array}$ & $\begin{array}{r}10 \\
N \\
10 \\
<10 \\
30\end{array}$ & $\begin{array}{r}7 \\
10 \\
50 \\
10 \\
50\end{array}$ & $\begin{array}{r}N \\
N \\
70 \\
70 \\
<50\end{array}$ & $\begin{array}{r}7 \\
10 \\
<5 \\
<5 \\
20\end{array}$ & $\begin{array}{r}20 \\
N \\
<20 \\
<20 \\
N\end{array}$ & $\begin{array}{r}<5 \\
5 \\
50 \\
7 \\
10\end{array}$ & $\begin{array}{r}N \\
200 \\
50 \\
1,000 \\
300\end{array}$ \\
\hline $\begin{array}{l}80 H 3532 \\
8 D H 3533 \\
80 H 3534 \\
80 H 3535 \\
80 H 3536\end{array}$ & $\begin{array}{l}10 \\
20 \\
15 \\
15 \\
15\end{array}$ & $\begin{array}{l}150 \\
700 \\
500 \\
700 \\
200\end{array}$ & $\begin{array}{c}N \\
<1.0 \\
N \\
N\end{array}$ & $\begin{array}{l}N \\
N \\
N \\
N \\
N\end{array}$ & $\begin{array}{l}N \\
N \\
N \\
N \\
N\end{array}$ & $\begin{array}{r}N \\
15 \\
<10 \\
<10 \\
<10\end{array}$ & $\begin{array}{r}30 \\
20 \\
<10 \\
10 \\
10\end{array}$ & $\begin{array}{l}20 \\
20 \\
15 \\
10 \\
10\end{array}$ & $\begin{array}{r}<50 \\
<50 \\
<50 \\
<50 \\
N\end{array}$ & $\begin{array}{l}5 \\
20 \\
10 \\
<5 \\
<5\end{array}$ & $\begin{array}{r}<20 \\
<20 \\
N \\
N \\
N\end{array}$ & $\begin{array}{r}<5 \\
20 \\
7 \\
5 \\
5\end{array}$ & $\begin{array}{r}150 \\
30 \\
50 \\
20 \\
100\end{array}$ \\
\hline $\begin{array}{l}8013537 \\
8013538 \\
8013539 \\
8013540 \\
8013541\end{array}$ & $\begin{array}{r}15 \\
15 \\
10 \\
<10 \\
<10\end{array}$ & $\begin{array}{r}100 \\
2,000 \\
300 \\
500 \\
50\end{array}$ & $\begin{array}{c}N \\
<1.0 \\
<1.0 \\
N \\
N\end{array}$ & $\begin{array}{r}N \\
20 \\
N \\
N \\
N\end{array}$ & $\begin{array}{l}N \\
N \\
N \\
N \\
N\end{array}$ & $\begin{array}{r}<10 \\
<10 \\
N \\
N \\
N\end{array}$ & $\begin{array}{r}<10 \\
15 \\
70 \\
N \\
N\end{array}$ & $\begin{array}{l}10 \\
15 \\
30 \\
10 \\
<5\end{array}$ & $\begin{array}{r}N \\
N \\
<50 \\
<50 \\
N\end{array}$ & $\begin{array}{r}<5 \\
10 \\
10 \\
<5 \\
N\end{array}$ & $\begin{array}{r}N \\
<20 \\
N \\
N \\
N\end{array}$ & $\begin{array}{r}5 \\
15 \\
20 \\
5 \\
<5\end{array}$ & $\begin{array}{r}20 \\
200 \\
150 \\
10 \\
N\end{array}$ \\
\hline $\begin{array}{l}8013542 \\
8013543 \\
8013544 \\
8013545 \\
8013546\end{array}$ & $\begin{array}{r}15 \\
20 \\
100 \\
15 \\
10\end{array}$ & $\begin{array}{r}1,000 \\
700 \\
500 \\
200 \\
100\end{array}$ & $\begin{array}{r}<1.0 \\
<1.0 \\
7.0 \\
<1.0 \\
N\end{array}$ & $\begin{array}{l}N \\
N \\
N \\
N \\
N\end{array}$ & $\begin{array}{l}N \\
N \\
N \\
N \\
N\end{array}$ & $\begin{array}{r}N \\
N \\
10 \\
N \\
N\end{array}$ & $\begin{array}{r}N \\
N \\
<10 \\
50 \\
<10\end{array}$ & $\begin{array}{r}5 \\
<5 \\
5 \\
10 \\
20\end{array}$ & $\begin{array}{r}100 \\
N \\
N \\
N \\
N\end{array}$ & $\begin{array}{r}<5 \\
<5 \\
<5 \\
5 \\
<5\end{array}$ & $\begin{array}{r}<20 \\
N \\
N \\
<20 \\
N\end{array}$ & $\begin{array}{l}N \\
7 \\
20 \\
<5 \\
<5\end{array}$ & $\begin{array}{r}200 \\
300 \\
50 \\
50 \\
N\end{array}$ \\
\hline
\end{tabular}


TABLE 4--RESULTS OF ANALYSES, DETROIT MINING DISTRICT, UTAH--Continued

\begin{tabular}{|c|c|c|c|c|c|c|c|c|c|c|c|c|}
\hline Sample & $\begin{array}{c}\text { Sb-ppm } \\
\text { s }\end{array}$ & $\begin{array}{c}\text { Sc-ppm } \\
\text { s }\end{array}$ & $\begin{array}{l}\text { Sn-ppm } \\
\text { s }\end{array}$ & $\begin{array}{c}\text { Sr-ppm } \\
\mathrm{s}\end{array}$ & $\begin{array}{c}\text { V-ppm } \\
\text { s }\end{array}$ & $\begin{array}{c}W-p p m \\
s\end{array}$ & $\underset{\mathbf{s}}{Y-p p m}$ & $\begin{array}{c}\text { 2n-ppm } \\
s\end{array}$ & $\begin{array}{c}z r-p p m \\
s\end{array}$ & $\begin{array}{c}\text { Th-ppm } \\
\text { s }\end{array}$ & $\begin{array}{c}\text { Au-ppm } \\
\text { aa }\end{array}$ & $\begin{array}{l}\text { AS-PPM } \\
\text { ICP }\end{array}$ \\
\hline $\begin{array}{l}9 \mathrm{dh} 3493 \mathrm{~h} \\
80 \mathrm{H} 3500 \\
8013501 \\
8013502 \\
8013503\end{array}$ & $\begin{array}{l}N \\
N \\
N \\
N \\
N\end{array}$ & $\begin{array}{r}N \\
N \\
<5 \\
N \\
N\end{array}$ & $\begin{array}{l}N \\
N \\
N \\
N \\
N\end{array}$ & $\begin{array}{r}150 \\
N \\
N \\
N \\
N\end{array}$ & $\begin{array}{r}70 \\
<10 \\
150 \\
50 \\
15\end{array}$ & $\begin{array}{l}N \\
N \\
N \\
N \\
N\end{array}$ & $\begin{array}{r}N \\
N \\
10 \\
N \\
N\end{array}$ & $\begin{array}{l}N \\
N \\
N \\
N \\
N\end{array}$ & $\begin{array}{r}70 \\
50 \\
100 \\
50 \\
150\end{array}$ & $\begin{array}{l}N \\
N \\
N \\
N \\
N\end{array}$ & $\begin{array}{l}.25 \\
N \\
N \\
N \\
N\end{array}$ & $\begin{array}{r}23.0 \\
<5.0 \\
170.0 \\
17.0 \\
110.0\end{array}$ \\
\hline $\begin{array}{l}8013504 \\
80 H 3505 \\
80 H 3506 \\
80 H 3507 \\
80 H 3508\end{array}$ & $\begin{array}{l}N \\
N \\
N \\
N \\
N\end{array}$ & $\begin{array}{l}N \\
N \\
N \\
N \\
N\end{array}$ & $\begin{array}{l}N \\
N \\
N \\
N \\
N\end{array}$ & $\begin{array}{r}<100 \\
N \\
100 \\
N \\
N\end{array}$ & $\begin{array}{l}20 \\
50 \\
15 \\
15 \\
10\end{array}$ & $\begin{array}{l}N \\
N \\
N \\
N \\
N\end{array}$ & $\begin{array}{l}N \\
N \\
N \\
N \\
N\end{array}$ & $\begin{array}{l}N \\
N \\
N \\
N \\
N\end{array}$ & $\begin{array}{r}150 \\
300 \\
50 \\
100 \\
50\end{array}$ & $\begin{array}{l}N \\
N \\
N \\
N \\
N\end{array}$ & $\begin{array}{l}N \\
N \\
.05 \\
N \\
.05\end{array}$ & $\begin{array}{l}47.0 \\
50.0 \\
51.0 \\
80.0 \\
28.0\end{array}$ \\
\hline $\begin{array}{l}80 h 3510 \\
80 h 3511 \\
80 h 3512 \\
80 h 3513 \\
80 h 3514\end{array}$ & $\begin{array}{l}N \\
N \\
N \\
N \\
N\end{array}$ & $\begin{array}{r}N \\
N \\
N \\
<5 \\
15\end{array}$ & $\begin{array}{l}N \\
N \\
N \\
N \\
N\end{array}$ & $\begin{array}{r}N \\
500 \\
N \\
1,000 \\
<100\end{array}$ & $\begin{array}{r}15 \\
50 \\
30 \\
100 \\
20\end{array}$ & $\begin{array}{l}N \\
N \\
N \\
N \\
N\end{array}$ & $\begin{array}{r}N \\
N \\
N \\
N \\
70\end{array}$ & $\begin{array}{l}N \\
N \\
N \\
N \\
N\end{array}$ & $\begin{array}{l}150 \\
150 \\
100 \\
100 \\
200\end{array}$ & $\begin{array}{l}N \\
N \\
N \\
N \\
N\end{array}$ & $\begin{array}{l}N \\
.05 \\
.10 \\
.10 \\
.30\end{array}$ & $\begin{array}{r}<5.0 \\
19.0 \\
27.0 \\
330.0 \\
3,700.0\end{array}$ \\
\hline $\begin{array}{l}80 H 3515 \\
80 H 3516 \\
80 h 3517 \\
8013518 \\
80 h 3519\end{array}$ & $\begin{array}{r}N \\
N \\
N \\
<100 \\
N\end{array}$ & $\begin{array}{l}N \\
N \\
N \\
N \\
5\end{array}$ & $\begin{array}{l}N \\
N \\
N \\
N \\
N\end{array}$ & $\begin{array}{r}700 \\
<100 \\
N \\
N \\
<100\end{array}$ & $\begin{array}{r}15 \\
15 \\
15 \\
50 \\
500\end{array}$ & $\begin{array}{r}N \\
N \\
N \\
20 \\
N\end{array}$ & $\begin{array}{r}N \\
15 \\
<10 \\
N \\
10\end{array}$ & $\begin{array}{r}N \\
N \\
N \\
N \\
700\end{array}$ & $\begin{array}{r}70 \\
300 \\
300 \\
200 \\
200\end{array}$ & $\begin{array}{l}N \\
N \\
N \\
N \\
N\end{array}$ & $\begin{array}{l}N \\
N \\
N \\
N \\
N\end{array}$ & $\begin{array}{r}71.0 \\
70.0 \\
18.0 \\
120.0 \\
89.0\end{array}$ \\
\hline $\begin{array}{l}80 H 3520 \\
80 H 3521 \\
80 H 3522 \\
80 H 3523 \\
80 H 3524\end{array}$ & $\begin{array}{r}N \\
N \\
N \\
N \\
100\end{array}$ & $\begin{array}{l}5 \\
5 \\
5 \\
5 \\
7\end{array}$ & $\begin{array}{l}N \\
N \\
N \\
N \\
N\end{array}$ & $\begin{array}{r}500 \\
700 \\
N \\
700 \\
1,000\end{array}$ & $\begin{array}{r}200 \\
150 \\
20 \\
100 \\
100\end{array}$ & $\begin{array}{l}N \\
N \\
N \\
N \\
N\end{array}$ & $\begin{array}{l}15 \\
10 \\
15 \\
15 \\
20\end{array}$ & $\begin{array}{r}N \\
N \\
N \\
1,000 \\
200\end{array}$ & $\begin{array}{l}300 \\
200 \\
500 \\
300 \\
200\end{array}$ & $\begin{array}{l}N \\
N \\
N \\
N \\
N\end{array}$ & $\begin{array}{l}N \\
N \\
N \\
.05 \\
N\end{array}$ & $\begin{array}{r}150.0 \\
14.0 \\
<5.0 \\
150.0 \\
310.0\end{array}$ \\
\hline $\begin{array}{l}80 H 3526 \\
8013527 \\
80 H 3528 \\
80 H 3529 \\
80 H 3530\end{array}$ & $\begin{array}{r}N \\
500 \\
N \\
N \\
N\end{array}$ & $\begin{array}{r}7 \\
5 \\
10 \\
5 \\
5\end{array}$ & $\begin{array}{l}N \\
N \\
N \\
N \\
N\end{array}$ & $\begin{array}{r}N \\
200 \\
<100 \\
200 \\
200\end{array}$ & $\begin{array}{r}20 \\
100 \\
30 \\
70 \\
100\end{array}$ & $\begin{array}{r}N \\
50 \\
N \\
N \\
N\end{array}$ & $\begin{array}{l}20 \\
10 \\
20 \\
15 \\
20\end{array}$ & $\begin{array}{l}N \\
N \\
N \\
N \\
N\end{array}$ & $\begin{array}{l}300 \\
100 \\
200 \\
200 \\
200\end{array}$ & $\begin{array}{l}N \\
N \\
N \\
N \\
N\end{array}$ & $\begin{array}{l}N \\
.40 \\
N \\
N \\
N\end{array}$ & $\begin{array}{r}13.0 \\
370.0 \\
20.0 \\
13.0 \\
20.0\end{array}$ \\
\hline $\begin{array}{l}80 H 3532 \\
80 H 3533 \\
80 H 3534 \\
80 H 3535 \\
80 H 3536\end{array}$ & $\begin{array}{r}300 \\
N \\
N \\
N \\
N\end{array}$ & $\begin{array}{l}<5 \\
<5 \\
N \\
5 \\
N\end{array}$ & $\begin{array}{l}N \\
N \\
N \\
N \\
N\end{array}$ & $\begin{array}{r}<100 \\
150 \\
500 \\
300 \\
500\end{array}$ & $\begin{array}{l}30 \\
50 \\
30 \\
50 \\
50\end{array}$ & $\begin{array}{l}N \\
N \\
N \\
N \\
N\end{array}$ & $\begin{array}{r}10 \\
15 \\
N \\
20 \\
<10\end{array}$ & $\begin{array}{l}N \\
N \\
N \\
N \\
N\end{array}$ & $\begin{array}{l}200 \\
300 \\
150 \\
200 \\
100\end{array}$ & $\begin{array}{l}N \\
N \\
N \\
N \\
N\end{array}$ & $\begin{array}{l}N \\
N \\
N \\
N \\
N\end{array}$ & $\begin{array}{r}48.0 \\
47.0 \\
13.0 \\
8.0 \\
14.0\end{array}$ \\
\hline $\begin{array}{l}8013537 \\
8013538 \\
8013539 \\
8013540 \\
8013541\end{array}$ & $\begin{array}{l}N \\
N \\
N \\
N \\
N\end{array}$ & $\begin{array}{l}N \\
7 \\
5 \\
5 \\
N\end{array}$ & $\begin{array}{l}N \\
N \\
N \\
N \\
N\end{array}$ & $\begin{array}{r}500 \\
300 \\
700 \\
1,000 \\
N\end{array}$ & $\begin{array}{r}50 \\
100 \\
100 \\
100 \\
50\end{array}$ & $\begin{array}{r}N \\
30 \\
N \\
N \\
N\end{array}$ & $\begin{array}{r}N \\
20 \\
<10 \\
<10 \\
N\end{array}$ & $\begin{array}{r}N \\
N \\
200 \\
N \\
N\end{array}$ & $\begin{array}{l}150 \\
200 \\
300 \\
200 \\
200\end{array}$ & $\begin{array}{l}N \\
N \\
N \\
N \\
N\end{array}$ & $\begin{array}{l}N \\
.10 \\
.20 \\
N \\
N\end{array}$ & $\begin{array}{r}42.0 \\
280.0 \\
89.0 \\
52.0 \\
15.0\end{array}$ \\
\hline $\begin{array}{l}8013542 \\
8013543 \\
8013544 \\
8013545 \\
8013546\end{array}$ & $\begin{array}{l}N \\
N \\
N \\
N \\
N\end{array}$ & $\begin{array}{r}10 \\
N \\
<5 \\
5 \\
5\end{array}$ & $\begin{array}{l}N \\
N \\
N \\
N \\
N\end{array}$ & $\begin{array}{r}1,000 \\
1,000 \\
700 \\
700 \\
150\end{array}$ & $\begin{array}{l}100 \\
100 \\
200 \\
150 \\
100\end{array}$ & $\begin{array}{l}N \\
N \\
N \\
N \\
N\end{array}$ & $\begin{array}{r}20 \\
<10 \\
10 \\
10 \\
30\end{array}$ & $\begin{array}{l}N \\
N \\
N \\
N \\
N\end{array}$ & $\begin{array}{r}500 \\
70 \\
100 \\
300 \\
300\end{array}$ & $\begin{array}{l}N \\
N \\
N \\
N \\
N\end{array}$ & $\begin{array}{l}\dot{N}^{10} \\
N \\
N \\
N\end{array}$ & $\begin{array}{r}100.0 \\
55.0 \\
240.0 \\
18.0 \\
44.0\end{array}$ \\
\hline
\end{tabular}


TABLE 4--RESULTS OF ANALYSES, DETROIT MINING DISTRICT, UTAH--Continued

\begin{tabular}{|c|c|c|c|c|c|c|c|c|c|c|}
\hline Sample & $\begin{array}{l}\text { BI-PPM } \\
\text { ICP }\end{array}$ & $\begin{array}{l}C D-P P M \\
I C P\end{array}$ & $\begin{array}{l}\text { SB-PPM } \\
\text { ICP }\end{array}$ & $\begin{array}{l}Z N-P P M \\
I C P\end{array}$ & $\begin{array}{c}\mathrm{Na}-\mathrm{pct} \\
\mathrm{s}\end{array}$ & $\begin{array}{c}\text { P-pct. } \\
\text { s }\end{array}$ & $\begin{array}{c}\text { Ga-ppm } \\
\text { s }\end{array}$ & $\begin{array}{l}\text { Ge-ppm } \\
\text { s }\end{array}$ & $\begin{array}{l}\text { HG-PPM } \\
\mathrm{CV}\end{array}$ & $\begin{array}{l}\text { F\% } \\
\text { ISE }\end{array}$ \\
\hline $\begin{array}{l}9 \mathrm{dh} 3493 \mathrm{~h} \\
80 \mathrm{H} 3500 \\
8013501 \\
8013502 \\
8013503\end{array}$ & $\begin{array}{r}1.50 \\
<2.00 \\
<2.00 \\
<2.00 \\
<2.00\end{array}$ & $\begin{array}{r}.033 \\
<.100 \\
.500 \\
.300 \\
.400\end{array}$ & $\begin{array}{r}12.00 \\
3.00 \\
12.00 \\
4.00 \\
3.00\end{array}$ & $\begin{array}{r}6.30 \\
<2.00 \\
35.00 \\
3.00 \\
7.00\end{array}$ & $\begin{array}{l}N \\
N \\
N \\
N \\
N\end{array}$ & $\begin{array}{l}N \\
<.2 \\
<.2 \\
N \\
N\end{array}$ & $\begin{array}{r}10 \\
N \\
10 \\
<5 \\
5\end{array}$ & $\begin{array}{l}N \\
N \\
N \\
N \\
N\end{array}$ & $\begin{array}{l}.18 \\
.36 \\
. N \\
N\end{array}$ & $\begin{array}{l}.03 \\
N \\
N \\
N \\
N\end{array}$ \\
\hline $\begin{array}{l}8013504 \\
80 H 3505 \\
80 H 3506 \\
80 H 3507 \\
80 H 3508\end{array}$ & $\begin{array}{l}<2.00 \\
<2.00 \\
<2.00 \\
<2.00 \\
<2.00\end{array}$ & $\begin{array}{l}.200 \\
.500 \\
.400 \\
.700 \\
.300\end{array}$ & $\begin{array}{l}2.00 \\
3.00 \\
3.00 \\
2.00 \\
7.00\end{array}$ & $\begin{array}{r}<2.00 \\
9.00 \\
12.00 \\
9.00 \\
<2.00\end{array}$ & $\begin{array}{l}N \\
N \\
N \\
N \\
N\end{array}$ & $\begin{array}{l}<.2 \\
N \\
<.2 \\
<.2 \\
N\end{array}$ & $\begin{array}{r}5 \\
5 \\
5 \\
5 \\
<5\end{array}$ & $\begin{array}{l}\mathrm{N} \\
\mathrm{N} \\
\mathrm{N} \\
\mathrm{N} \\
\mathrm{N}\end{array}$ & $\begin{array}{l}.32 \\
.24 \\
.10 \\
.10 \\
.14\end{array}$ & $\begin{array}{l}N \\
N \\
.05 \\
N \\
.05\end{array}$ \\
\hline $\begin{array}{l}80 H 3510 \\
80 H 3511 \\
80 H 3512 \\
80 H 3513 \\
80 H 3514\end{array}$ & $\begin{array}{r}<2.00 \\
4.00 \\
7.00 \\
4.00 \\
<2.00\end{array}$ & $\begin{array}{r}.200 \\
.300 \\
1.000 \\
.900 \\
2.300\end{array}$ & $\begin{array}{r}<2.00 \\
10.00 \\
21.00 \\
5.00 \\
22.00\end{array}$ & $\begin{array}{r}<2.00 \\
4.00 \\
28.00 \\
4.00 \\
61.00\end{array}$ & $\begin{array}{l}N \\
N \\
N \\
<.2 \\
<.2\end{array}$ & $\begin{array}{l}<.2 \\
<.2 \\
N \\
N_{N}\end{array}$ & $\begin{array}{r}<5 \\
5 \\
7 \\
10 \\
10\end{array}$ & $\begin{array}{l}N \\
N \\
N \\
N \\
N\end{array}$ & $\begin{array}{l}.04 \\
.04 \\
.04 \\
.20 \\
.0\end{array}$ & $\begin{array}{l}N \\
.05 \\
.10 \\
.10 \\
.30\end{array}$ \\
\hline $\begin{array}{l}80 H 3515 \\
80 H 3516 \\
80 H 3517 \\
8013518 \\
80 H 3519\end{array}$ & $\begin{array}{l}<2.00 \\
<2.00 \\
<2.00 \\
<2.00 \\
<2.00\end{array}$ & $\begin{array}{r}.200 \\
<.100 \\
<.100 \\
.100 \\
1.300\end{array}$ & $\begin{array}{r}5.00 \\
3.00 \\
<2.00 \\
58.00 \\
4.00\end{array}$ & $\begin{array}{r}36.00 \\
8.00 \\
4.00 \\
12.00 \\
320.00\end{array}$ & $\begin{array}{l}N \\
N \\
N \\
N \\
N\end{array}$ & $\begin{array}{l}<.2 \\
<.2 \\
N \\
N \\
<.2\end{array}$ & $\begin{array}{l}<5 \\
<5 \\
<5 \\
<5 \\
10\end{array}$ & $\begin{array}{l}N \\
N \\
N \\
N \\
N\end{array}$ & $\begin{array}{l}N \\
.06 \\
.02 \\
.02 \\
.18\end{array}$ & $\begin{array}{l}N \\
<.01 \\
<.01 \\
<.01 \\
.03\end{array}$ \\
\hline $\begin{array}{l}80 H 3520 \\
80 H 3521 \\
80 H 3522 \\
80 H 3523 \\
80 H 3524\end{array}$ & $\begin{array}{r}<2.00 \\
<2.00 \\
<2.00 \\
7.00 \\
<2.00\end{array}$ & $\begin{array}{r}.200 \\
.600 \\
<.100 \\
1.100 \\
.700\end{array}$ & $\begin{array}{r}4.00 \\
<2.00 \\
<2.00 \\
18.00 \\
86.00\end{array}$ & $\begin{array}{r}69.00 \\
55.00 \\
8.00 \\
610.00 \\
160.00\end{array}$ & $\begin{array}{l}N \\
N \\
N \\
N \\
N\end{array}$ & $\begin{array}{r}.2 \\
.2 \\
<.2 \\
<.2 \\
.2\end{array}$ & $\begin{array}{l}<5 \\
20 \\
<5 \\
10 \\
30\end{array}$ & $\begin{array}{l}N \\
N \\
N \\
N \\
N\end{array}$ & $\begin{array}{l}.12 \\
.02 \\
.08 \\
.32 \\
1.30\end{array}$ & $\begin{array}{r}.03 \\
.08 \\
<.01 \\
.03 \\
.05\end{array}$ \\
\hline $\begin{array}{l}8 D H 3526 \\
8 D 13527 \\
8 D H 3528 \\
8 D H 3529 \\
8 D H 3530\end{array}$ & $\begin{array}{r}<2.00 \\
<2.00 \\
3.00 \\
<2.00 \\
<2.00\end{array}$ & $\begin{array}{l}<.100 \\
2.000 \\
.200 \\
.200 \\
.500\end{array}$ & $\begin{array}{r}5.00 \\
270.00 \\
5.00 \\
<2.00 \\
3.00\end{array}$ & $\begin{array}{r}7.00 \\
41.00 \\
18.00 \\
27.00 \\
77.00\end{array}$ & $\begin{array}{l}N \\
N \\
N \\
N \\
N\end{array}$ & $\begin{array}{r}<.2 \\
<.2 \\
<.2 \\
.2 \\
<.2\end{array}$ & $\begin{array}{r}N \\
10 \\
N \\
20 \\
15\end{array}$ & $\begin{array}{r}N \\
20 \\
N \\
N \\
N\end{array}$ & $\begin{array}{r}.16 \\
.38 \\
.12 \\
1.80 \\
.06\end{array}$ & $\begin{array}{l}<.01 \\
<.01 \\
<.01 \\
.02 \\
.02\end{array}$ \\
\hline $\begin{array}{l}80 H 3532 \\
80 H 3533 \\
8 D H 3534 \\
8 D H 3535 \\
8 D H 3536\end{array}$ & $\begin{array}{r}7.00 \\
4.00 \\
<2.00 \\
<2.00 \\
<2.00\end{array}$ & $\begin{array}{l}.200 \\
.700 \\
.400 \\
.200 \\
.100\end{array}$ & $\begin{array}{r}49.00 \\
4.00 \\
<2.00 \\
<2.00 \\
<2.00\end{array}$ & $\begin{array}{r}3.00 \\
92.00 \\
18.00 \\
6.00 \\
2.00\end{array}$ & $\begin{array}{l}N \\
N \\
N \\
N \\
N\end{array}$ & $\begin{array}{l}<.2 \\
N \\
.2 \\
.5 \\
<.2\end{array}$ & $\begin{array}{l}N \\
5 \\
5 \\
5 \\
7\end{array}$ & $\begin{array}{l}N \\
N \\
N \\
N \\
N\end{array}$ & $\begin{array}{r}3.80 \\
.04 \\
.10 \\
.04 \\
.04\end{array}$ & $\begin{array}{l}<.01 \\
<.01 \\
.02 \\
.02 \\
.01\end{array}$ \\
\hline $\begin{array}{l}8013537 \\
8013538 \\
8013539 \\
8013540 \\
8013541\end{array}$ & $\begin{array}{l}<2.00 \\
29.00 \\
<2.00 \\
<2.00 \\
<2.00\end{array}$ & $\begin{array}{r}.100 \\
.500 \\
.300 \\
.200 \\
<.100\end{array}$ & $\begin{array}{r}5.00 \\
40.00 \\
84.00 \\
6.00 \\
<2.00\end{array}$ & $\begin{array}{r}7.00 \\
170.00 \\
120.00 \\
9.00 \\
<2.00\end{array}$ & $\begin{array}{l}N \\
N \\
<.2 \\
N \\
N\end{array}$ & $\begin{array}{l}<.2 \\
<.2 \\
<.2 \\
<.2 \\
N\end{array}$ & $\begin{array}{r}<5 \\
5 \\
10 \\
<5 \\
<5\end{array}$ & $\begin{array}{l}N \\
N \\
N \\
N \\
N\end{array}$ & $\begin{array}{l}N \\
.16 \\
.10 \\
N \\
.02\end{array}$ & $\begin{array}{r}.01 \\
<.01 \\
.02 \\
.03 \\
<.01\end{array}$ \\
\hline $\begin{array}{l}8013542 \\
8013543 \\
8013544 \\
8013545 \\
8013546\end{array}$ & $\begin{array}{r}<2.00 \\
<2.00 \\
<2.00 \\
4.00 \\
<2.00\end{array}$ & $\begin{array}{r}.200 \\
.400 \\
1.400 \\
.500 \\
.100\end{array}$ & $\begin{array}{l}<2.00 \\
10.00 \\
13.00 \\
<2.00 \\
<2.00\end{array}$ & $\begin{array}{l}<2.00 \\
2.00 \\
9.00 \\
3.00 \\
6.00\end{array}$ & $\begin{array}{r}.2 \\
<.2 \\
.3 \\
<.2 \\
<.2\end{array}$ & $\begin{array}{l}.2 \\
<.2 \\
N \\
<.2 \\
<.2\end{array}$ & $\begin{array}{l}30 \\
10 \\
20 \\
10 \\
<5\end{array}$ & $\begin{array}{l}N \\
N \\
N \\
N \\
N\end{array}$ & $\begin{array}{r}2.80 \\
.08 \\
.06 \\
.02 \\
.04\end{array}$ & $\begin{array}{l}.04 \\
.03 \\
.03 \\
.02 \\
.01\end{array}$ \\
\hline
\end{tabular}


TABLE 4--RESULTS OF ANALYSES, DETROIT MINING DISTRICT, UTAH--Continued

\begin{tabular}{|c|c|c|c|c|c|c|c|c|c|c|}
\hline Sample & Latitude & Lnngitude & $\begin{array}{c}\text { Fe-pct } \\
\text { s }\end{array}$ & $\begin{array}{c}M g-p c t \\
s\end{array}$ & $\begin{array}{c}\text { Ca-pct. } \\
s\end{array}$ & $\underset{s}{T i-p c t .}$ & $\begin{array}{c}\text { Mn-ppm } \\
s\end{array}$ & $\underset{S}{A g-p p m}$ & $\begin{array}{c}\text { As - ppm } \\
s\end{array}$ & $\begin{array}{c}A u-p p m \\
s\end{array}$ \\
\hline $\begin{array}{l}80 ! 3547 \\
8013549 \\
8013549 \\
8013550 \\
8013551\end{array}$ & $\begin{array}{rrr}39 & 32 & 31 \\
39 & 32 & 0 \\
39 & 32 & 1 \\
39 & 31 & 56 \\
39 & 32 & 19\end{array}$ & $\begin{array}{lll}112 & 59 & 53 \\
112 & 59 & 22 \\
112 & 59 & 24 \\
112 & 59 & 37 \\
112 & 59 & 29\end{array}$ & $\begin{array}{r}1.50 \\
3.00 \\
2.00 \\
5.00 \\
10.00\end{array}$ & $\begin{array}{l}.03 \\
.20 \\
.15 \\
.15 \\
.20\end{array}$ & $\begin{array}{l}.20 \\
.20 \\
.50 \\
.20 \\
.15\end{array}$ & $\begin{array}{l}.200 \\
.300 \\
.300 \\
.500 \\
.500\end{array}$ & $\begin{array}{r}30 \\
50 \\
50 \\
20 \\
200\end{array}$ & $\begin{array}{l}N \\
N \\
N \\
N \\
N\end{array}$ & $\begin{array}{r}\mathbf{N} \\
10,000 \\
N \\
N \\
N\end{array}$ & $\begin{array}{l}N \\
N \\
N \\
N \\
N\end{array}$ \\
\hline $\begin{array}{l}8013552 \\
80 ! 3553 \\
8013554 \\
8013555 \\
80 ! 3556\end{array}$ & $\begin{array}{lll}39 & 31 & 43 \\
39 & 31 & 37 \\
39 & 31 & 34 \\
39 & 31 & 33 \\
39 & 31 & 28\end{array}$ & $\begin{array}{lll}112 & 59 & 47 \\
112 & 59 & 52 \\
112 & 59 & 58 \\
112 & 59 & 55 \\
112 & 59 & 52\end{array}$ & $\begin{array}{r}7.00 \\
10.00 \\
7.00 \\
15.00 \\
5.00\end{array}$ & $\begin{array}{r}.03 \\
.20 \\
.20 \\
.30 \\
1.50\end{array}$ & $\begin{array}{l}.10 \\
.20 \\
.70 \\
5.00 \\
1.00\end{array}$ & $\begin{array}{l}.050 \\
.300 \\
.100 \\
.050 \\
.070\end{array}$ & $\begin{array}{r}20 \\
100 \\
150 \\
500 \\
>5,000\end{array}$ & $\dot{N}_{N}^{N} .70$ & $\begin{array}{r}500 \\
1,500 \\
700 \\
1,500 \\
700\end{array}$ & $\begin{array}{l}\mathrm{N} \\
\mathrm{N} \\
\mathrm{N} \\
\mathrm{N} \\
\mathrm{N}\end{array}$ \\
\hline $\begin{array}{l}80 H 3557 \\
80 H 3558 \\
80 H 3559\end{array}$ & $\begin{array}{lll}39 & 31 & 31 \\
39 & 31 & 33 \\
39 & 31 & 45\end{array}$ & $\begin{array}{lll}113 & 0 & 0 \\
113 & 0 & 0 \\
113 & 0 & 2\end{array}$ & $\begin{array}{r}20.00 \\
.10 \\
2.00\end{array}$ & $\begin{array}{r}.20 \\
<.02 \\
.20\end{array}$ & $\begin{array}{r}3.00 \\
.05 \\
.20\end{array}$ & $\begin{array}{l}.070 \\
.050 \\
.200\end{array}$ & $\begin{array}{r}200 \\
20 \\
50\end{array}$ & $\begin{array}{c}<.50 \\
\mathrm{~N}\end{array}$ & $\begin{array}{r}500 \\
N \\
N\end{array}$ & $\begin{array}{l}N \\
N \\
N\end{array}$ \\
\hline
\end{tabular}


TABLE 4--RESULTS UF ANALYSES, DETROIY MINING DISTRICT, UTAH--Continued

\begin{tabular}{|c|c|c|c|c|c|c|c|c|c|c|c|c|c|}
\hline Sample & $\begin{array}{c}\text { B-ppm } \\
\text { s }\end{array}$ & $\begin{array}{c}\mathrm{Ba}-\mathrm{ppm} \\
\mathrm{s}\end{array}$ & $\begin{array}{c}\text { Be-ppm } \\
\text { s }\end{array}$ & $\begin{array}{c}\text { Ei-ppm } \\
\mathrm{s}\end{array}$ & $\begin{array}{c}\mathrm{Cd}-\mathrm{ppm} \\
\mathrm{s}\end{array}$ & $\begin{array}{c}\text { Co-ppm } \\
\mathrm{s}\end{array}$ & $\begin{array}{c}\mathrm{Cr} \text {-ppm } \\
\mathrm{s}\end{array}$ & $\begin{array}{c}\text { Cu-ppm } \\
s\end{array}$ & $\begin{array}{l}\text { La-ppm } \\
\mathrm{s}\end{array}$ & $\begin{array}{c}\text { Mo-ppm } \\
\mathrm{s}\end{array}$ & $\begin{array}{l}\text { Nb-ppm } \\
s\end{array}$ & $\begin{array}{c}\mathrm{Ni} \text {-ppm } \\
\mathrm{s}\end{array}$ & $\begin{array}{c}\text { Pb-ppm } \\
s\end{array}$ \\
\hline $\begin{array}{l}8013547 \\
8013548 \\
8013549 \\
8013550 \\
8013551\end{array}$ & $\begin{array}{r}10 \\
30 \\
30 \\
100 \\
70\end{array}$ & $\begin{array}{r}500 \\
700 \\
1,000 \\
150 \\
1,000\end{array}$ & $\begin{array}{r}<1.0 \\
5.0 \\
1.0 \\
<1.0 \\
2.0\end{array}$ & $\begin{array}{l}N \\
N \\
N \\
N \\
N\end{array}$ & $\begin{array}{l}\mathbf{N} \\
\mathbf{N} \\
\mathbf{N} \\
\mathbf{N} \\
\mathbf{N}\end{array}$ & $\begin{array}{r}<10 \\
30 \\
<10 \\
10 \\
20\end{array}$ & $\begin{array}{r}<10 \\
100 \\
10 \\
20 \\
150\end{array}$ & $\begin{array}{l}30 \\
15 \\
10 \\
20 \\
15\end{array}$ & $\begin{array}{r}<50 \\
50 \\
<50 \\
<50 \\
50\end{array}$ & $\begin{array}{l}10 \\
10 \\
<5 \\
15 \\
N\end{array}$ & $\begin{array}{l}N \\
N \\
N \\
N \\
N\end{array}$ & $\begin{array}{r}10 \\
20 \\
5 \\
20 \\
15\end{array}$ & $\begin{array}{r}50 \\
30 \\
<10 \\
20 \\
50\end{array}$ \\
\hline $\begin{array}{l}8013552 \\
8013553 \\
8013554 \\
8013555 \\
8013556\end{array}$ & $\begin{array}{r}20 \\
50 \\
50 \\
<10 \\
50\end{array}$ & $\begin{array}{l}200 \\
200 \\
100 \\
<20 \\
200\end{array}$ & $\begin{array}{r}2.0 \\
1.0 \\
3.0 \\
<9.0 \\
5.0\end{array}$ & $\begin{array}{l}N \\
N \\
N \\
N \\
N\end{array}$ & $\begin{array}{l}N \\
N \\
N \\
N \\
N\end{array}$ & $\begin{array}{l}N \\
N \\
20 \\
15 \\
70\end{array}$ & $\begin{array}{r}<10 \\
<10 \\
50 \\
20 \\
\mathrm{~N}\end{array}$ & $\begin{array}{r}15 \\
10 \\
15 \\
100 \\
100\end{array}$ & $\begin{array}{r}N \\
50 \\
N \\
N \\
N\end{array}$ & $\begin{array}{l}10 \\
30 \\
15 \\
10 \\
20\end{array}$ & $\begin{array}{l}\mathbf{N} \\
\mathbf{N} \\
\mathbf{N} \\
\mathbf{N} \\
\mathbf{N}\end{array}$ & $\begin{array}{r}7 \\
5 \\
15 \\
20 \\
150\end{array}$ & $\begin{array}{r}10 \\
50 \\
50 \\
500 \\
200\end{array}$ \\
\hline $\begin{array}{l}80 H 3557 \\
80 H 3558 \\
80 H 3559\end{array}$ & $\begin{array}{l}20 \\
20 \\
50\end{array}$ & $\begin{array}{r}50 \\
20 \\
200\end{array}$ & $\begin{array}{l}<1.0 \\
<1.0 \\
<1.0\end{array}$ & $\begin{array}{l}N \\
N \\
N\end{array}$ & $\begin{array}{l}\mathrm{N} \\
\mathrm{N} \\
\mathrm{N}\end{array}$ & $\begin{array}{r}20 \\
N \\
<10\end{array}$ & $\begin{array}{l}N \\
20 \\
50\end{array}$ & $\begin{array}{l}70 \\
<5 \\
20\end{array}$ & $\begin{array}{r}<50 \\
N \\
<50\end{array}$ & $\begin{array}{l}5 \\
N \\
7\end{array}$ & $\begin{array}{r}N \\
N \\
<20\end{array}$ & $\begin{array}{r}50 \\
<5 \\
7\end{array}$ & $\begin{array}{r}200 \\
N \\
200\end{array}$ \\
\hline
\end{tabular}


TABLE ४--RESULTS OF ANALYSES, DETROII MIN!NG DISTRICT, UTAH--CONt inued

\begin{tabular}{|c|c|c|c|c|c|c|c|c|c|c|c|c|}
\hline Sample & $\begin{array}{c}\text { Sb-ppm } \\
\text { s }\end{array}$ & $\begin{array}{c}\text { Sc-ppm } \\
\mathrm{s}\end{array}$ & $\begin{array}{c}\text { Sn-ppm } \\
s\end{array}$ & $\begin{array}{c}\text { Sr-ppm } \\
\text { s }\end{array}$ & $\begin{array}{c}v-p p m \\
s\end{array}$ & $\begin{array}{c}\text { W-ppm } \\
\text { s }\end{array}$ & $\begin{array}{c}Y-p p m \\
s\end{array}$ & $\begin{array}{c}2 n-p p m \\
s\end{array}$ & $\begin{array}{c}2 r-p p m \\
s\end{array}$ & $\begin{array}{c}\text { Th-ppm } \\
s\end{array}$ & $\begin{array}{c}\text { Au-ppm } \\
\text { aa }\end{array}$ & $\begin{array}{l}\text { AS-PPM } \\
\text { ICP }\end{array}$ \\
\hline $\begin{array}{l}8013547 \\
8013548 \\
8013549 \\
8013550 \\
8013551\end{array}$ & $\begin{array}{l}N \\
N \\
N \\
N \\
N\end{array}$ & $\begin{array}{r}10 \\
15 \\
7 \\
15 \\
20\end{array}$ & $\begin{array}{l}N \\
N \\
N \\
N \\
N\end{array}$ & $\begin{array}{l}500 \\
500 \\
100 \\
200 \\
300\end{array}$ & $\begin{array}{l}150 \\
150 \\
100 \\
150 \\
150\end{array}$ & $\begin{array}{l}N \\
N \\
N \\
N \\
N\end{array}$ & $\begin{array}{l}20 \\
30 \\
20 \\
20 \\
30\end{array}$ & $\begin{array}{l}N \\
N \\
N \\
N \\
N\end{array}$ & $\begin{array}{l}300 \\
200 \\
300 \\
200 \\
200\end{array}$ & $\begin{array}{l}N \\
N \\
N \\
N \\
N\end{array}$ & $\begin{array}{l}N \\
N \\
N \\
N \\
N\end{array}$ & $\begin{array}{r}62.0 \\
3.600 .0 \\
32.0 \\
28.0 \\
67.0\end{array}$ \\
\hline $\begin{array}{l}8013552 \\
8013553 \\
8013554 \\
8013555 \\
8013556\end{array}$ & $\begin{array}{r}\mathbf{N} \\
\mathbf{N} \\
\mathbf{N} \\
200 \\
\mathbf{N}\end{array}$ & $\begin{array}{r}5 \\
7 \\
5 \\
<5 \\
<5\end{array}$ & $\begin{array}{l}N \\
N \\
N \\
N \\
N\end{array}$ & $\begin{array}{r}500 \\
200 \\
<100 \\
300 \\
700\end{array}$ & $\begin{array}{r}150 \\
50 \\
70 \\
100 \\
150\end{array}$ & $\begin{array}{l}N \\
N \\
N \\
N \\
N\end{array}$ & $\begin{array}{r}10 \\
100 \\
50 \\
15 \\
100\end{array}$ & $\begin{array}{r}N \\
N \\
N \\
200 \\
500\end{array}$ & $\begin{array}{r}70 \\
200 \\
200 \\
70 \\
70\end{array}$ & $\begin{array}{l}N \\
N \\
N \\
N \\
N\end{array}$ & $\begin{array}{l}\mathrm{N} \\
\mathrm{N} \\
\mathrm{N} \\
.50 \\
.05\end{array}$ & $\begin{array}{r}490.0 \\
820.0 \\
470.0 \\
1.600 .0 \\
570.0\end{array}$ \\
\hline $\begin{array}{l}80 H 3557 \\
80 H 3558 \\
80 H 3559\end{array}$ & $\begin{array}{l}N \\
N \\
N\end{array}$ & $\begin{array}{r}<5 \\
N \\
10\end{array}$ & $\begin{array}{l}N \\
N \\
N\end{array}$ & $\begin{array}{r}200 \\
N \\
300\end{array}$ & $\begin{array}{r}200 \\
10 \\
100\end{array}$ & $\begin{array}{l}N \\
N \\
N\end{array}$ & $\begin{array}{r}20 \\
<10 \\
20\end{array}$ & $\begin{array}{l}N \\
N \\
N\end{array}$ & $\begin{array}{r}50 \\
100 \\
200\end{array}$ & $\begin{array}{l}\mathrm{N} \\
\mathrm{N} \\
\mathrm{N}\end{array}$ & $\begin{array}{l}N \\
<.05 \\
<.05\end{array}$ & $\begin{array}{r}360.0 \\
300.0 \\
58.0\end{array}$ \\
\hline
\end{tabular}


TABLE 4--RESULTS JF ANALYSES, DETROIT MINING DISTRICT, UTAH--CONt inued

\begin{tabular}{|c|c|c|c|c|c|c|c|c|c|c|}
\hline Sample & $\begin{array}{l}\text { BI-PPM } \\
\text { ICP }\end{array}$ & $\begin{array}{l}\text { CD-PPM } \\
\text { ICP }\end{array}$ & $\begin{array}{l}\text { SB-PPM } \\
I C P\end{array}$ & $\begin{array}{l}Z N-P P M \\
I C P\end{array}$ & $\begin{array}{c}\mathrm{Na}-\mathrm{pct} \\
\mathrm{s}\end{array}$ & $\begin{array}{c}p-p c t . \\
s\end{array}$ & $\begin{array}{c}\text { Ga-ppm } \\
s\end{array}$ & $\begin{array}{c}\text { Ge-ppm } \\
\text { s }\end{array}$ & $\begin{array}{l}\text { HG-PPM } \\
\mathrm{CV}\end{array}$ & $\begin{array}{l}\text { F\% } \\
\text { ISE }\end{array}$ \\
\hline $\begin{array}{l}8013547 \\
8013548 \\
8013549 \\
8013550 \\
8013551\end{array}$ & $\begin{array}{l}<2.00 \\
<2.00 \\
<2.00 \\
<2.00 \\
<2.00\end{array}$ & $\begin{array}{r}.200 \\
.300 \\
.200 \\
.400 \\
1.100\end{array}$ & $\begin{array}{l}<2.00 \\
39.00 \\
<2.00 \\
2.00 \\
3.00\end{array}$ & $\begin{array}{r}15.00 \\
14.00 \\
4.00 \\
4.00 \\
21.00\end{array}$ & $\begin{array}{l}<.2 \\
<.2 \\
<.2 \\
.2 \\
<.2\end{array}$ & $\begin{array}{r}.2 \\
.3 \\
.2 \\
.3 \\
<.2\end{array}$ & $\begin{array}{l}15 \\
20 \\
20 \\
30 \\
50\end{array}$ & $\begin{array}{l}N \\
N \\
N \\
N \\
N\end{array}$ & $\begin{array}{l}.08 \\
.0 \\
N \\
\dot{N}^{4}\end{array}$ & $\begin{array}{l}.03 \\
.03 \\
.03 \\
.07 \\
.07\end{array}$ \\
\hline $\begin{array}{l}8013552 \\
8013553 \\
8013554 \\
8013555 \\
8013556\end{array}$ & $\begin{array}{r}<2.00 \\
<2.00 \\
<2.00 \\
4.00 \\
<2.00\end{array}$ & $\begin{array}{r}.400 \\
.900 \\
1.300 \\
5.300 \\
4.100\end{array}$ & $\begin{array}{r}10.00 \\
5.00 \\
7.00 \\
120.00 \\
20.00\end{array}$ & $\begin{array}{r}22.00 \\
3.00 \\
81.00 \\
57.00 \\
490.00\end{array}$ & $\begin{array}{r}<.2 \\
.2 \\
<.2 \\
<.2 \\
1.0\end{array}$ & $\begin{array}{l}.5 \\
N \\
.2 \\
.5\end{array}$ & $\begin{array}{r}5 \\
20 \\
10 \\
70 \\
20\end{array}$ & $\begin{array}{l}\text { N } \\
N \\
N \\
N \\
N\end{array}$ & $\begin{array}{l}.10 \\
N \\
.32 \\
.16 \\
.18\end{array}$ & $\begin{array}{l}<.01 \\
.02 \\
.03 \\
.02 \\
.04\end{array}$ \\
\hline $\begin{array}{l}80 H 3557 \\
80 H 3558 \\
80 H 3559\end{array}$ & $\begin{array}{l}10.00 \\
<2.00 \\
<2.00\end{array}$ & $\begin{array}{r}6.000 \\
7.700 \\
.200\end{array}$ & $\begin{array}{r}7.00 \\
27.00 \\
<2.00\end{array}$ & $\begin{array}{r}39.00 \\
410.00 \\
12.00\end{array}$ & $\begin{array}{l}<.2 \\
<.2 \\
<.2\end{array}$ & $\begin{array}{c}N \\
<.2 \\
N\end{array}$ & $\begin{array}{r}30 \\
5 \\
50\end{array}$ & $\begin{array}{l}\text { N } \\
N \\
N\end{array}$ & $\begin{array}{l}.14 \\
.10 \\
.04\end{array}$ & $\begin{array}{l}<.01 \\
.03 \\
.05\end{array}$ \\
\hline
\end{tabular}

